\title{
Les matériaux de construction en terre cuite d'époque romaine dans l'ouest des Pays de la Loire - Premier bilan
}

Roman terracotta construction materials from the West of the Pays de la Loire- a first assessment

Los materiales de construcción de terracota de época romana en el oeste de los Pays de la Loire : primeros resultados

Jean-François Nauleau

\section{OpenEdition}

Journals

Édition électronique

URL : https://journals.openedition.org/rao/2154

DOI : 10.4000/rao.2154

ISBN : 978-2-7535-3432-2

ISSN : $1775-3732$

Éditeur

Presses universitaires de Rennes

\section{Édition imprimée}

Date de publication : 25 décembre 2013

Pagination : 223-259

ISBN : 978-2-7535-3430-8

ISSN : 0767-709X

Référence électronique

Jean-François Nauleau, « Les matériaux de construction en terre cuite d'époque romaine dans l'ouest des Pays de la Loire - Premier bilan », Revue archéologique de l'Ouest [En ligne], 30 | 2013, mis en ligne le 25 décembre 2015, consulté le 19 août 2022. URL : http://journals.openedition.org/rao/2154; DOI https://doi.org/10.4000/rao.2154

Ce document a été généré automatiquement le 19 août 2022.

Tous droits réservés 


\title{
Les matériaux de construction en terre cuite d'époque romaine dans l'ouest des Pays de la Loire - Premier bilan
}

\author{
Roman terracotta construction materials from the West of the Pays de la Loire- a \\ first assessment \\ Los materiales de construcción de terracota de época romana en el oeste de los \\ Pays de la Loire : primeros resultados
}

Jean-François Nauleau

Je tiens à remercier toutes les personnes qui ont contribué à l'aboutissement de ce travail, en particulier M.-H. Santrot (musée départemental Thomas Dobrée, Nantes) ainsi que D. Legouestre (SRA Pays de la Loire), O. de Peretti (archéologue municipal, Ville de Rezé), L. Pirault (Inrap Grand-Ouest), D. Séris (Inrap Grand-Ouest), A. Polinski (doctorant, université de Nantes), V. Poinsignon (Inrap Grand-Ouest), F. Mercier (Direction du patrimoine et de l'archéologie, Nantes), A. Archer (étudiant en master 2 « Métiers de l'archéologie »), R. Jemin (Inrap Grand-Ouest), S. Thébaud (Inrap GrandOuest).

1 Les matériaux de construction en terre cuite tiennent une place primordiale dans la construction en Gaule romaine. Employés dans les maçonneries, dans les édifices balnéaires et sur les toitures, ils deviennent au cours du $\mathrm{I}^{\text {er }}$ siècle apr. J.-C. des matériaux incontournables. Les maçonneries en opus mixtum des enceintes des chefslieux de cité dont les décors polychromes du Mans ou encore du mausolée de CinqMars-la-Pile, employant de grandes quantités de briques, sont même devenus des édifices emblématiques de cette période.

2 Sur les sites, même en l'absence de vestiges d'élévations maçonnées, les restes de matériaux en terre cuite sont abondants. Pourtant, les études les concernant sont très rares. Ces matériaux sont considérés comme marqueurs chronologiques de la période 
romaine mais ne sont que très rarement l'objet d'études propres. Plusieurs raisons expliquent un tel désintérêt. La variabilité typologique de ces matériaux est largement sous-estimée, seuls les matériaux de couverture - tegulae et imbrices - étant la plupart du temps identifiés. Ils sont le plus souvent très fragmentés et donc difficilement identifiables sur le terrain, et ont pour inconvénient d'être lourds et volumineux, donc complexes à gérer sur le terrain et en phase d'étude. Par ailleurs, le format de ces matériaux paraît très normé pour l'ensemble du monde romain. En résumé, il semble aller de soi que ces matériaux ont peu à apporter à l'archéologue.

Pourtant, ces dernières années, quelques études ont montré tout l'intérêt qu'il y avait à aborder ce thème de recherche, qu'il s'agisse de la grande variabilité typologique de ces matériaux notamment dans le domaine des thermes (Bouet, 1999), de l'évolution morphologique des tegulae dans le temps (Feugère, 2000 ; Warry, 2006 ; Clément, 2009), ou encore de la compréhension architecturale de leur mise en œuvre (Vipard, 1999).

L'article présenté ici est un premier bilan d'un travail débuté il y a maintenant trois ans dans la partie ouest des Pays de la Loire et qui a permis de constituer une abondante documentation, majoritairement issue d'opérations préventives récentes (tabl.1). Le but d'une telle étude est de faire progresser l'identification de ces matériaux et la compréhension de leur mise en contexte architectural. Cependant, on verra que bien d'autres thèmes sont abordés, tels que l'évolution morphologique de ces éléments, par exemple. Une base de données a été développée, permettant l'intégration et le traitement de tous leurs caractères morphologiques. À l'heure actuelle, plus de mille individus sont enregistrés dans la base, ce qui représente un effectif suffisant pour autoriser un premier bilan.

Tableau 1 : Liste de provenance des matériaux de terre cuite intégrés à l'étude. Table 1 : Liste of the provenance of terracotta materials studied

\begin{tabular}{|c|c|c|c|c|c|c|c|}
\hline Sites de provenance & Dépt. & $\begin{array}{l}\text { Nbre de } \\
\text { restes } \\
\text { étudiés }\end{array}$ & $\begin{array}{c}\text { Type } \\
\text { d'occupation }\end{array}$ & Chronologie & $\begin{array}{c}\text { Opération } \\
\text { archéologique }\end{array}$ & $\begin{array}{l}\text { Responsable } \\
\text { d'opération }\end{array}$ & $\begin{array}{c}\text { Date } \\
\text { d'intervention }\end{array}$ \\
\hline $\begin{array}{l}\text { Haute-Goulaine 'Les Cléons' } \\
\text { Haute-Goulaine 'Les Cléons' }\end{array}$ & $\begin{array}{l}44 \\
44\end{array}$ & 270 & $\begin{array}{l}\text { villa? } \\
\text { villa? }\end{array}$ & $\begin{array}{l}\text { T-Ille? } \\
\text { 1-Ille? }\end{array}$ & $\begin{array}{c}\text { Fouille } \\
\text { Diagnostic Inrap }\end{array}$ & F. Chaillou & $\begin{array}{l}\text { Fin XIXe } \\
2010\end{array}$ \\
\hline Rezé 'Rue Saint-lupien AH 77' & 44 & 6 & Quartier d'habitat & $1-v_{e}$ & Fouille Inrap & M.L. Hervé & 2006 \\
\hline Rezé 'quartier Saint-lupien' & 44 & 3 & Quartier d'habitat & I-Ve? & $\begin{array}{l}\text { Fouille Université- } \\
\text { Ville de Rezé- }\end{array}$ & $\begin{array}{l}\text { M. Monteil /O. } \\
\text { de Peretti }\end{array}$ & 2007-2011 \\
\hline Rezé 'La tannière' & 44 & 12 & Basilique & $\mathrm{Vle}$ & Fouille Inrap & L. Pirault & 2006 \\
\hline $\begin{array}{l}\text { Rezé 'depót municipal' } \\
\text { (operations 1980-2005) }\end{array}$ & 44 & 40 & Quartier d'habitat & I-Vle? & $\begin{array}{l}\text { Opérations } \\
\text { diverses }\end{array}$ & multiples & $1980-2005$ \\
\hline Rezé 'Terrain Peigné' & 44 & 15 & domus & I- IIIe? & Fouille & S. Deschamps & 1989 \\
\hline Piriac-sur-Mer 'Le Pladreau' & 44 & 90 & villa? & I-Vlle & Fouille Inrap & M.-L. Hervé & 2005 \\
\hline $\begin{array}{l}\text { Saint-Mars-du-Désert, RD } 148, \\
\text { diag. }\end{array}$ & 44 & 2 & Edifice cultuel? & He? & Diagnostic Inrap & J. Cornec & 2010 \\
\hline $\begin{array}{l}\text { Saint-Herblain, 'Villa des Preux' } \\
\text { Saint-Herblain, 'Zac Ar Mor' }\end{array}$ & $\begin{array}{l}44 \\
44\end{array}$ & $\begin{array}{l}8 \\
3\end{array}$ & $\begin{array}{l}\text { villa } \\
?\end{array}$ & $\begin{array}{l}\text { I-Vle } \\
\text { I-IVe }\end{array}$ & $\begin{array}{l}\text { Fouille état } \\
\text { Fouille Inrap }\end{array}$ & $\begin{array}{l}\text { D. Barraud } \\
\text { F. Mercier }\end{array}$ & $\begin{array}{l}1979 \\
2006\end{array}$ \\
\hline Arthon-en-Retz 'La Bonneterie' & 44 & 5 & $?$ & ? & Diagnostic Inrap & D. Séris & 2009 \\
\hline $\begin{array}{l}\text { Saint-Michel-Chef-Chef 'La } \\
\text { Pouplinière' }\end{array}$ & 44 & 13 & $\begin{array}{l}\text { pars rustica de } \\
\text { villa? }\end{array}$ & I - IIIe + HMA & Fouille Inrap & D. Doyen & 2010 \\
\hline Machecoul 'Zac Richebourg' & 44 & 3 & $?$ & GR ind. & Diagnostic Inrap & F. Mercier & 2008 \\
\hline Carquefou, 'La Haute Forêt II' & 44 & 40 & $?$ & GR et HMA & Fouille Inrap & A. Valais & 2010 \\
\hline Nantes, 'Zac Erdre-Porterie' & 44 & 20 & ? & H-llle & Diagnostic Inrap & J.-F. Nauleau & 2009 \\
\hline Nantes 'Musée T. Dobrée' & 44 & 26 & $?$ & $?$ & $\begin{array}{l}\text { Provenances } \\
\text { inconnues }\end{array}$ & 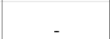 & $?$ \\
\hline Saint-Molf 'Rue de l'étang' & 44 & 41 & ? & GR ind. & Diagnostic Inrap & A. le Boulaire & 2009 \\
\hline $\begin{array}{l}\text { Couëron, 'Zac Les Hauts de } \\
\text { Couëron III' }\end{array}$ & 44 & 20 & $?$ & GR ind. & Diagnostic Inrap & K. Prêtre & 2011 \\
\hline Angers 'Marengo' & 49 & 74 & $\begin{array}{l}\text { quartier sub- } \\
\text { urbain? }\end{array}$ & I-NVe? & Fouille Inrap & M. Pithon & 2010 \\
\hline Angers 'Saint-Louis' & 49 & 179 & Ilot urbain & Hive & Fouille Inrap & J. Brodeur & 2010 \\
\hline $\begin{array}{l}\text { Beaufort-en-Vallée 'Chemin des } \\
\text { Airaults' }\end{array}$ & 49 & 2 & $?$ & GR ind. & Diagnostic Inrap & J. Toumeur & 2006 \\
\hline $\begin{array}{l}\text { Saint-Florent-le-Vieil, } \\
\text { 'Lotissement ouest' }\end{array}$ & 49 & 3 & villa? & GR ind. & Diagnostic Inrap & Y. Viau & 2012 \\
\hline $\begin{array}{c}\text { LGV 'secteur 13', La Cropte - } \\
\text { Bazougers' }\end{array}$ & 53 & 20 & $?$ & GR ind. & Diagnostic Inrap & C. Chauveau & 2011 \\
\hline Le Langon 'Rue de l'Industrie' & 85 & 5 & $\begin{array}{l}\text { agglomération } \\
\text { secondaire? }\end{array}$ & H-lie & Fouille Inrap & M.L. Hervè & 2007 \\
\hline Jard-sur-Mer 'Le Grand Essart' & 85 & 39 & $\begin{array}{l}\text { pars rustica de } \\
\text { villa }\end{array}$ & I-Ve? & Fouille Inrap & D. Séris & 2008 \\
\hline $\begin{array}{l}\text { Les Clouzeaux, 'La Grosse } \\
\text { Pierre' }\end{array}$ & 85 & 45 & $\begin{array}{l}\text { Atelier de } \\
\text { production TCA } \\
\text { et ceram. }\end{array}$ & I-llle & Fouille Inrap & D. Séris & 2007 \\
\hline $\begin{array}{l}\text { La Roche-sur-Yon, 'La Malboire } \\
\text { II' }\end{array}$ & 85 & 12 & 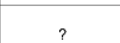 & GR ind. & Diagnostic Inrap & D. Séris & 2009 \\
\hline
\end{tabular}




\section{Identification des matériaux et mise en contexte architectural}

5 L'examen attentif des restes de matériaux en terre cuite révèle souvent leur grande variabilité typologique. Si, majoritairement, ils sont employés en couverture, une part non négligeable est constituée d'autres types d'éléments peu décrits dans la littérature. Pourtant, leur étude permet une mise en contexte architectural susceptible d'apporter de précieux renseignements à l'archéologue.

6 Les matériaux sont prévus au moment de leur fabrication pour un usage qui semble en théorie bien déterminé. Dans la pratique, les usages détournés de matériaux neufs sont cependant nombreux. Les tegulae, par exemple, sont souvent mises en œuvre dans des maçonneries de canalisations et les exemples sont abondants : Rezé, domus du « Terrain Peigné » (Deschamps et al., 1992) ; Angers «Saint-Louis » (Nauleau dans Brodeur, à paraître). Les parois de bacs à chaux sont très souvent constituées de tegulae: HauteGoulaine "Les Cléons ${ }^{1}$ ", Angers "Saint-Louis ». De même, les soles de four et de séchoir font fréquemment appel à ces matériaux: Rezé, "quartier Saint-Lupien » (Guitton et al., 2009) et domus du « Terrain Peigné ».

7 Les briques participent également de cette pratique: mentionnons les arases de bessales (briques carrées de pilettes d'hypocauste) insérées dans le mur conservé sous la chapelle Saint-Lupien à Rezé, ainsi que les briques de format quart-de-rond (destinées aux colonnes) de la canalisation de la domus du « Terrain Peigné » à Rezé. En outre, il faut prendre en considération les pratiques de remploi qui se manifestent abondamment durant l'Antiquité tardive et qui réaffectent alors des quantités considérables de matériaux (De Filippo, 2010).

\section{Les matériaux de couverture}

\section{Les tegulae}

\section{Morphologie}

8 Les tegulae sont des tuiles plates rectangulaires ou trapézoïdales munies d'un rebord sur les grands côtés. Elles sont pourvues d'encoches d'emboîtement en parties haute et basse $^{2}$ (fig. 1) qui permettent à la tuile aval de bloquer la tuile amont tout en assurant un recouvrement nécessaire à l'étanchéité (fig. 2). 
Figure 1 : Les encoches d'emboîtement des tegulae.

Figure 1 : Interlocking notches on tegulae

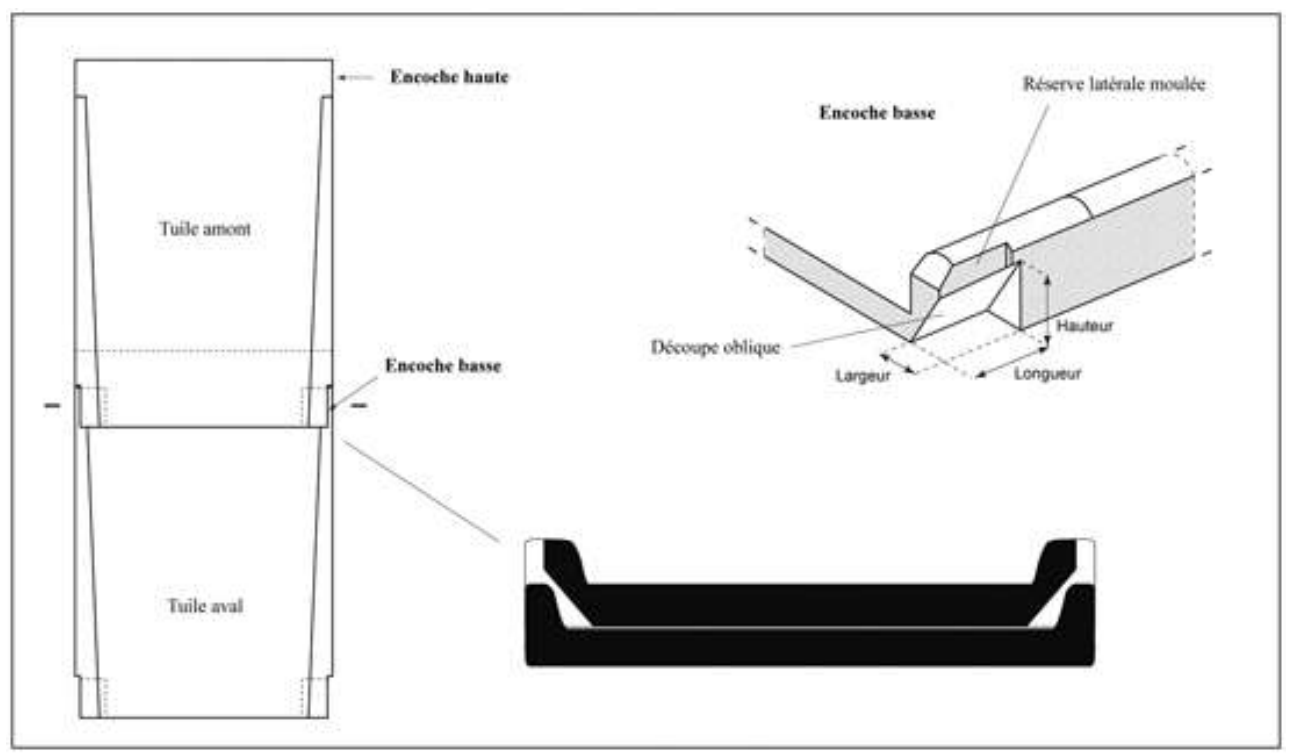

Figure 2 : L'emboîtement des tegulae.

Figure 2 : Interlocking of tegulae

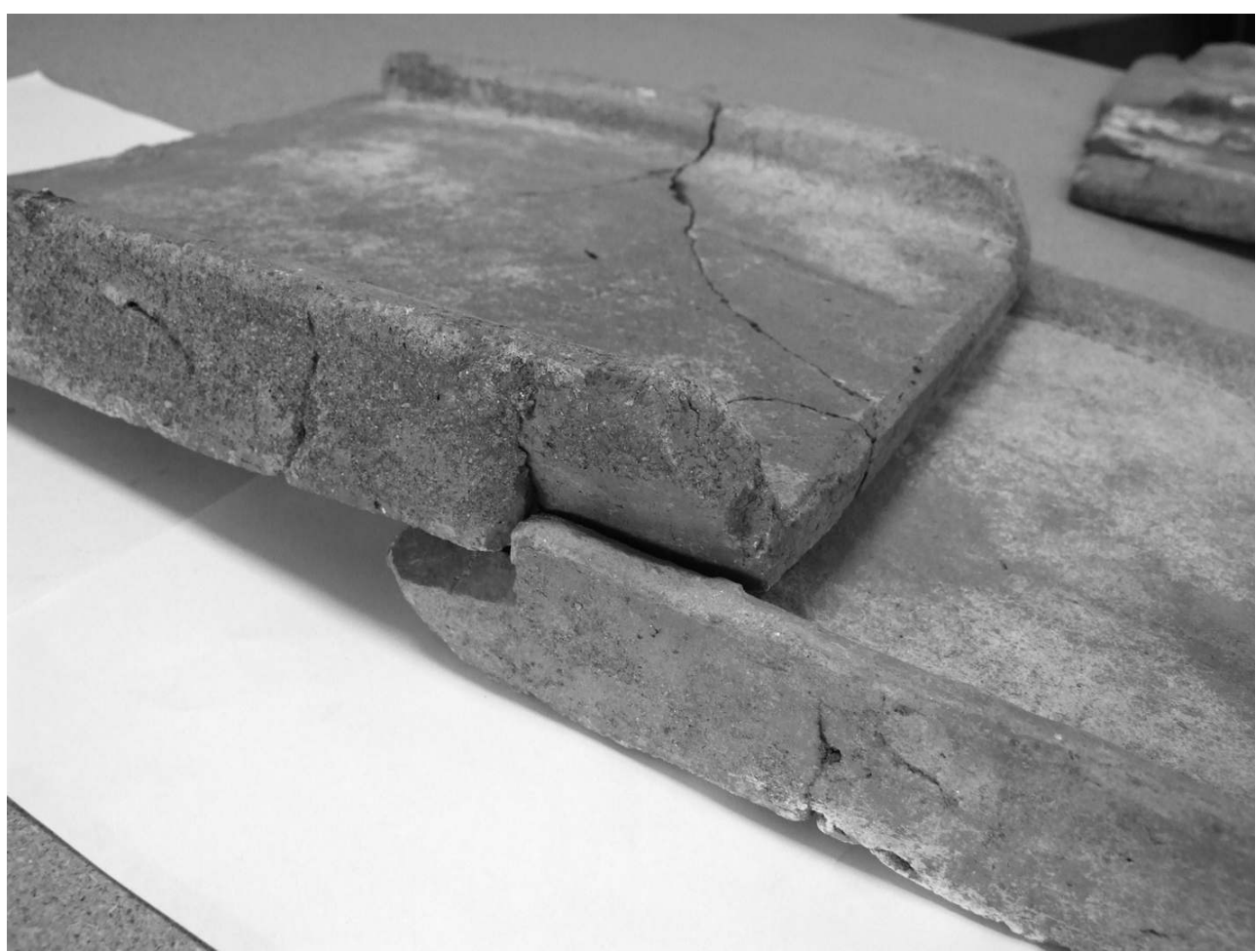

Cliché J.-F. Nauleau, Inrap.

9 La tegula romaine est un héritage de la culture technique grecque et hellénistique, mais les tuiles grecques étaient sensiblement différentes, les rebords étant moins marqués et constitués d'une simple inflexion du fond de la tuile. Elle est réalisée dans un moule en bois sans fond (forme) dont on sable les surfaces internes avant chaque façonnage, afin d'éviter l'adhérence de l'argile. Les parties externes - à savoir la face inférieure, les 
faces externes des rebords et les extrémités haute et basse - présentent ainsi une surface rugueuse liée à la présence de ce sable.

Les rebords ont des hauteurs stables entre parties haute et basse de la tuile. La largeur de ce rebord est en général nettement décroissante vers la partie haute, de façon à faciliter l'emboîtement de la tuile amont. Son épaisseur tend elle aussi à décroître vers la partie haute.

\section{L'emboîtement}

L'encoche haute montre les stigmates nets d'une découpe réalisée avec un outil tranchant et n'est qu'une simple découpe du rebord. En partie basse, l'encoche est plus complexe car constituée de deux parties bien distinctes. La première est une petite réserve dans la largeur du rebord, réalisée au moment du façonnage par l'adjonction d'une petite pièce de bois dans le moule. La seconde est une encoche oblique réalisée en face inférieure par découpe au couteau (fig.3). Elle est probablement réalisée au moment où les tuiles déjà indurées sont mises à la verticale pour faciliter leur séchage et libérer de la place sur l'aire de travail. Le recours à deux étapes est lié à l'impossibilité de réaliser une encoche de ce type par moulage seul.

Figure 3 : L'encoche d'emboîtement basse montrant les états de surface de la réserve latérale moulée et de la découpe oblique.

Figure 3 : Lower interlocking notches showing the surface of the lateral moulded reserve and the oblique cut.

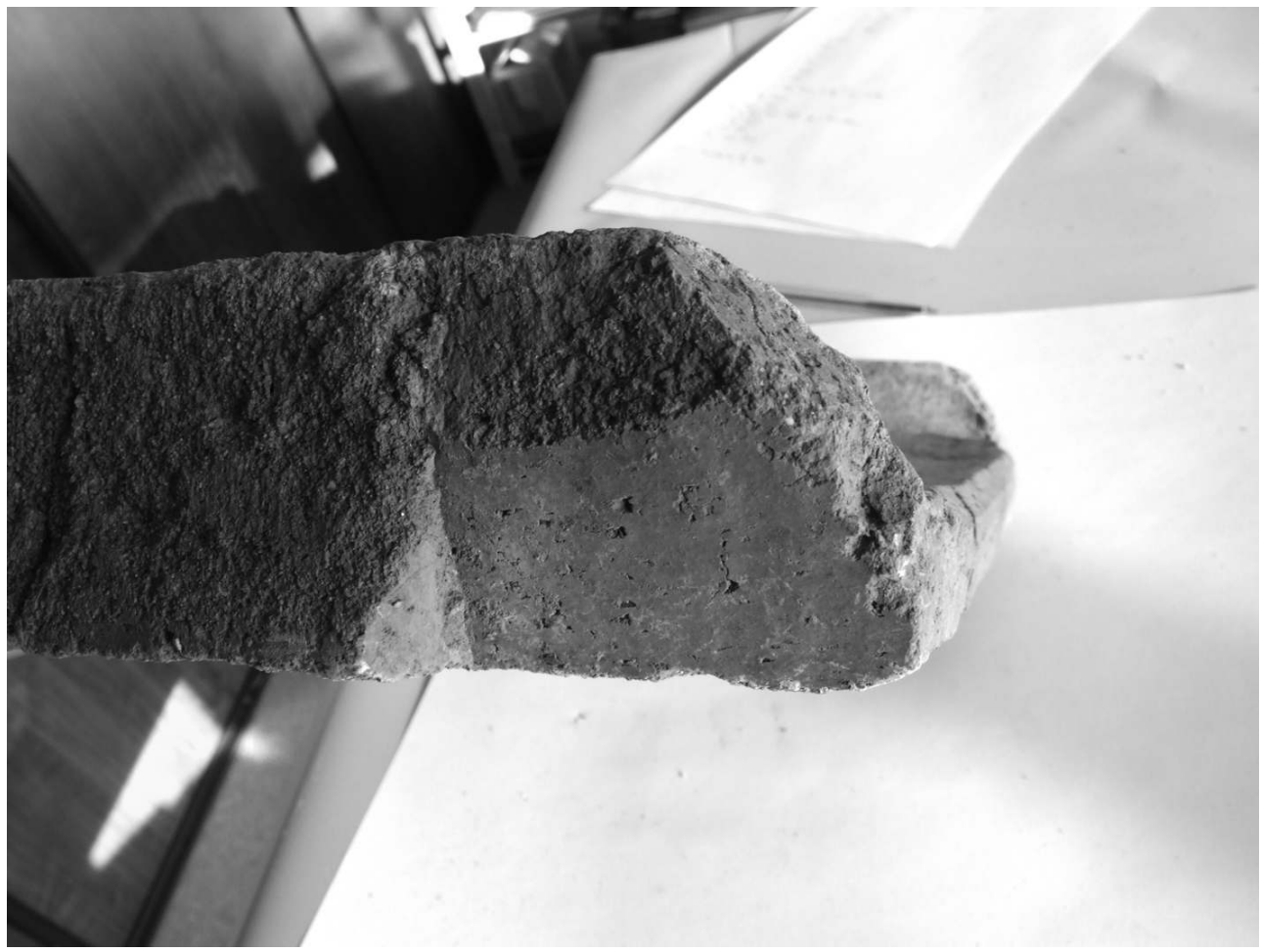

Cliché J.-F. Nauleau, Inrap.

En réalité, seule la découpe oblique est réellement nécessaire à l'emboitement, la réserve latérale n'étant utile que si le rebord de la tuile est très haut. Il semble que cette découpe latérale constitue un repère de longueur en préalable à la réalisation de la découpe oblique. Cette dernière est toujours sensiblement de même longueur que la 
réserve latérale. On verra plus loin tout l'intérêt d'une étude particulière des encoches d'emboîtement basses, dans la perspective d'une mise en évidence d'une évolution morphologique des tegulae (cf. infra).

L'assemblage sur la toiture se fait par un recouvrement dont la longueur est mesurable par addition des longueurs des encoches haute et basse de la tuile (fig.2). Ce recouvrement est par ailleurs visible dans certains cas sur les tegulae elles-mêmes, celles-ci montrant un état de surface très contrasté entre les parties protégées par le recouvrement et celles soumises aux intempéries et notamment le gel (fig. 4).

Figure 4 : L'état de surface des tegulae.

Figure 4 : Detail of the surface of the tegulae

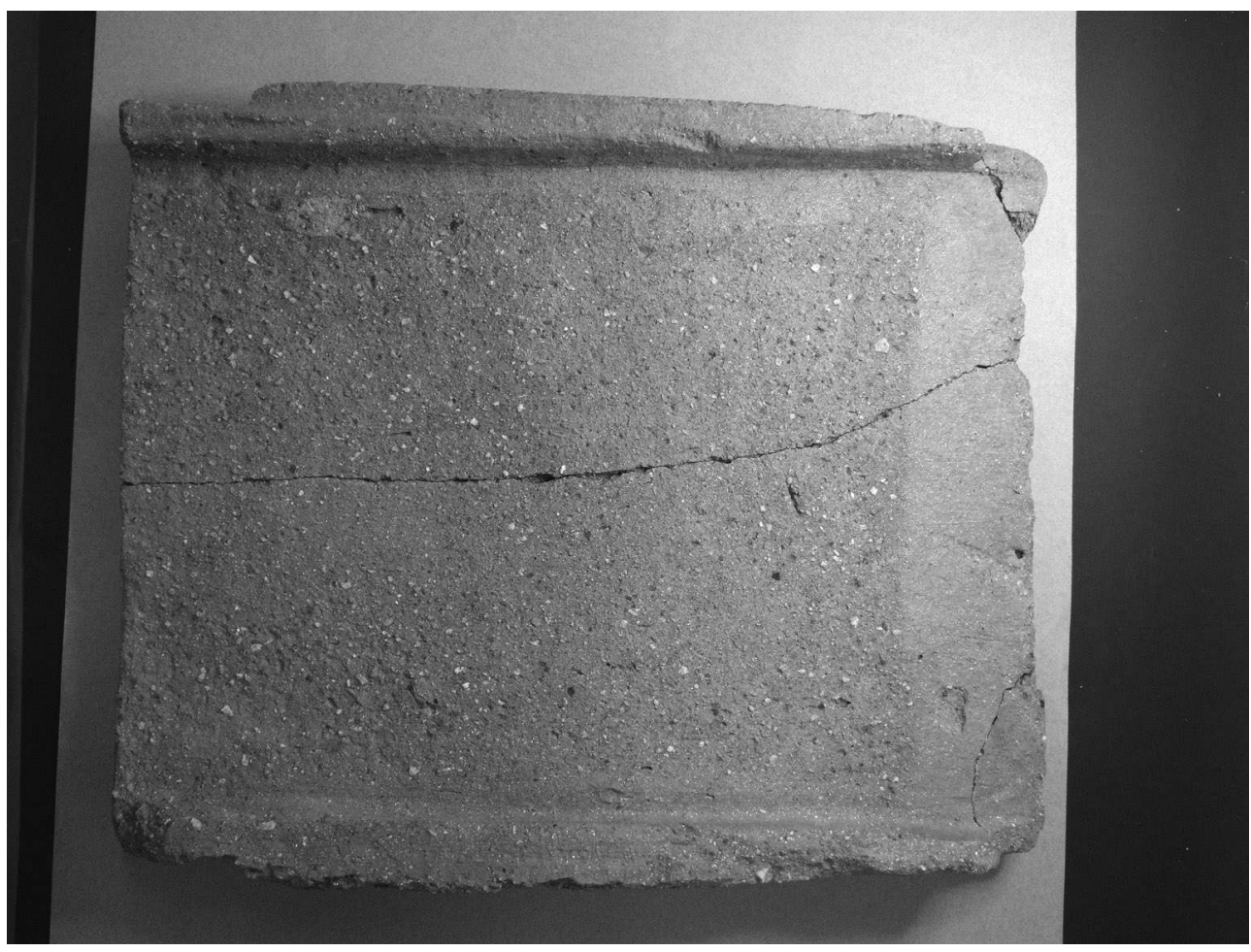

Cliché J.-F. Nauleau, Inrap.

\section{Pente des toitures, surface de pose et fixation}

On ne possède pas régionalement de témoignage archéologique concernant la pente des toitures. Cependant, ils sont relativement nombreux dans le monde romain. D'après toutes ces données, la pente semble comprise entre 17 et 23 degrés, soit entre 30 et $43 \%$ environ (Demandrille, Cambou, 2008, p. 4-5). La plupart des reconstitutions architecturales sont réalisées avec une pente de 20 degrés. Les données mécaniques confirment ces résultats car il est impossible que ces tuiles supportent, sans glissement, une pente supérieure à 25 degrés (Lyonnet, 2008, p. 24), sauf avec système d'accroche. Or ces tuiles ne sont munies morphologiquement d'aucun système d'accroche en face inférieure et les perforations de fixation restent très rares. Seules six tuiles sur les trois cents tegulae enregistrées possèdent une perforation fonctionnelle, c'est-à-dire réalisée avant cuisson en partie haute, dans la zone de recouvrement sous la tuile amont, ce qui permet d'une part de maintenir l'étanchéité de la couverture et d'autre part d'éviter la corrosion du clou. Quelques tuiles présentent des perforations en partie basse, toujours 
réalisées après cuisson. Elles étaient probablement utilisées en bas de toiture, à l'égout du toit, et permettaient de retenir, dans une certaine mesure, les files de tegulae.

Des reconstitutions de toiture permettant de mieux saisir toutes les implications techniques de ce type de matériau de couverture ont été réalisées (fig. 5). Elles confirment que la pente la plus probable se situe bien aux alentours de 20 degrés, une tendance au glissement étant constatée à partir de 25 degrés.

Figure 5 : Toiture en tegulae et imbrices reconstituée.

Figure 5 : Recreated roofing of tegulae and imbrices

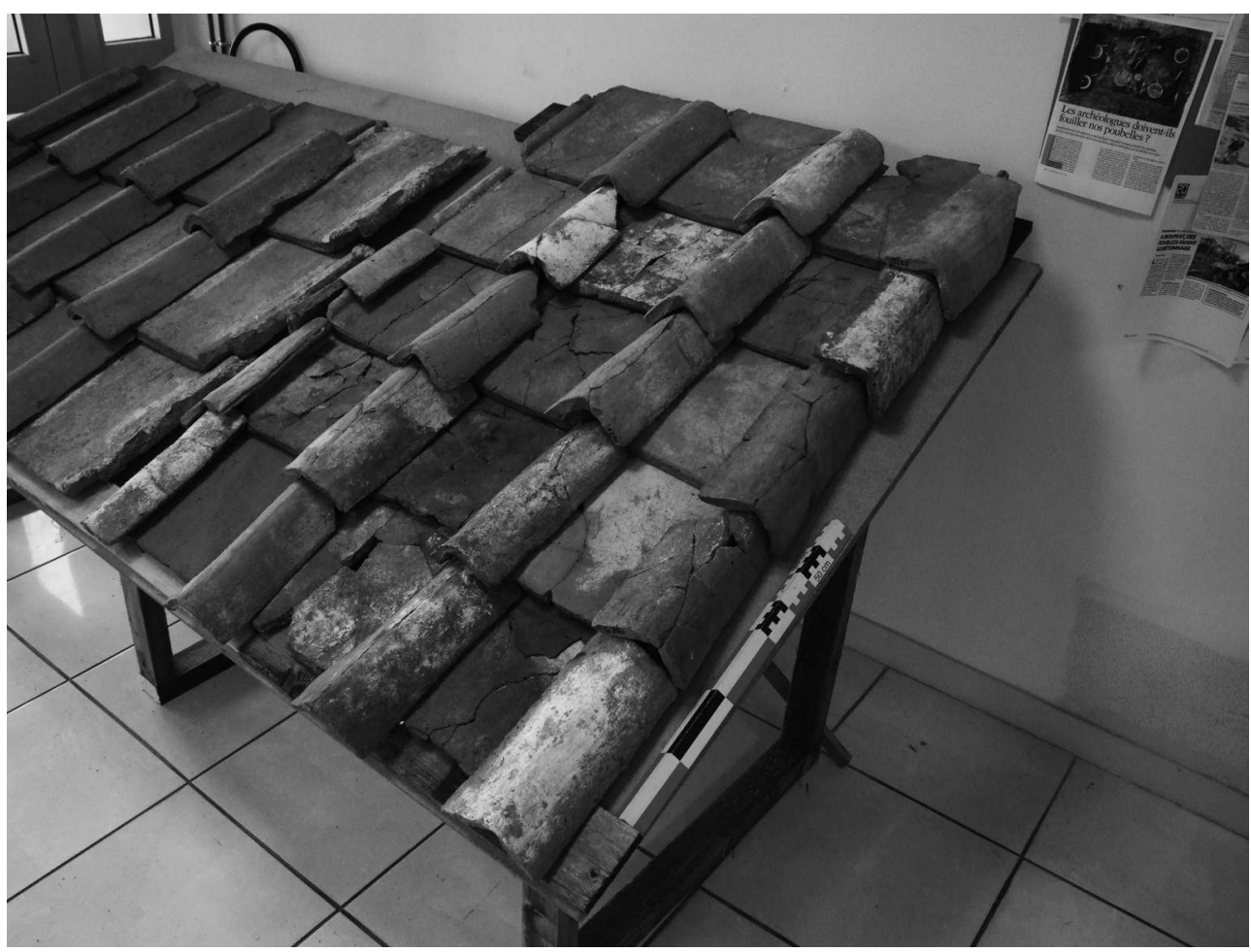

Cliché J.-F. Nauleau, Inrap.

Compte tenu de cette valeur de pente, la longueur du recouvrement constaté (entre 90 et $125 \mathrm{~mm}$ ) est faible et ne semble pas en mesure d'assurer une bonne étanchéité3. Pour des pentes inférieures à 25 degrés, l'usage de la tuile est de nos jours proscrit. Pour des pentes de 25 degrés, les recouvrements recommandés sont compris entre 14 et 17 centimètres, suivant les types de climat. Les faibles pentes de ce type ne supportent pas la conjugaison de la pluie et du vent qui cause des problèmes d'infiltration. On peut donc légitimement se poser la question de l'adéquation réelle de ces couvertures méditerranéennes aux climats atlantiques de la région d'étude.

\section{Poids des matériaux de couverture}

17 Le poids moyen au mètre carré d'une toiture réalisée en tegulae est simple à calculer à partir de tuiles complètes. L'opération consiste à calculer le nombre de tuiles au mètre carré puis de le multiplier par la masse d'une tegula et d'une imbrex (calcul ci-dessous). Pour déterminer ce nombre, on doit tenir compte de la longueur du recouvrement. Celui-ci est obtenu en additionnant les longueurs de l'encoche basse et de la découpe haute de la tuile. 


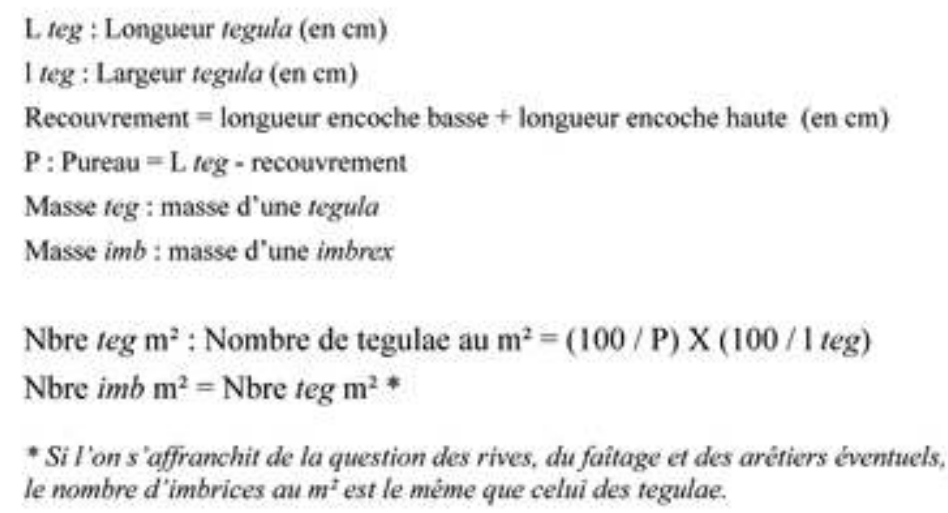

Masse de la toiture au $\mathrm{m}^{2}=\left(\right.$ Nbre $t e g \mathrm{~m}^{2} \mathrm{X}$ masse $\left.t e g\right)$ $+\left(\right.$ Nbre teg $\mathbf{m}^{2} \mathrm{X}$ masse $\mathrm{imb}$ )

La valeur finale obtenue ne tient pas compte de la présence éventuelle de boudins de mortiers sous les imbrices. Il occasionnera le calcul du nombre de mètre linéaire de mortier et l'estimation du poids d'un mètre linéaire.

Sans tenir compte de la question du mortier, les valeurs ainsi obtenues varient de $71 \mathrm{~kg}$ (toiture effondrée US 3206, Angers "Saint-Louis ») à près de $95 \mathrm{~kg} / \mathrm{m}^{2}$ (Haute-Goulaine "Les Cléons » et Angers "Saint-Louis" », US 3291) (cf. § 2). Ces valeurs doivent être comparées à celles connues pour les tuiles mécaniques actuelles, 35 à $45 \mathrm{~kg} / \mathrm{m}^{2}$, (Lyonnet, 2008, p. 15) - soit pratiquement la moitié - ainsi qu'aux valeurs concernant les tuiles « canal » médiévales et modernes ${ }^{4}$, environ $45 \mathrm{~kg} / \mathrm{m}^{2}$ (Nauleau, 2009a).

Cette masse importante est pénalisante car elle nécessite des bois de charpente de forte section et donc plus coûteux. Mais il semble, d'après les premiers résultats de cette étude, que cette donnée a évolué dans le temps (cf. § 2).

\section{Les imbrices ou couvre-joints}

21 Leur morphologie est identique à celle des tuiles "canal» que nous connaissons actuellement. La différence de largeur entre parties amont et aval semble moindre, mais la différence majeure concerne leur épaisseur qui atteint pour les imbrices romaines des valeurs très élevées, la moyenne pour les soixante-dix-neuf exemplaires enregistrés dans la base étant de $21 \mathrm{~mm}$ pour la partie aval et $23 \mathrm{~mm}$ pour la partie amont. Dès le Moyen Âge, ces épaisseurs chutent et atteignent des valeurs comparables à celles qui sont rencontrées actuellement. Le lot de tuiles «canal » à crochets mis au jour à Précigné en Sarthe ( $\mathrm{XIV}^{\mathrm{e}}-\mathrm{XV}^{\mathrm{e}}$ siècle), par exemple, montre des épaisseurs comprises entre 9 et $11 \mathrm{~mm}$ (ibid.). Le poids des imbrices enregistrées dans la base est très variable, de 1400 à $3300 \mathrm{~g}$, contre 1000 à $1200 \mathrm{~g}$ pour le lot médiéval de Précigné. Les longueurs sont comprises entre 295 et $440 \mathrm{~mm}$, mais les rares exemplaires supérieurs à $400 \mathrm{~mm}$ sont plutôt à rapporter à la catégorie des tuiles de faîtage, et la valeur moyenne constatée est de $347 \mathrm{~mm}$.

La longueur moyenne des imbrices est en général inférieure à celle rencontrée pour les tegulae associées. Ainsi, pour la toiture effondrée d'Angers "Saint-Louis ", la longueur (19 individus) est inférieure de $64 \mathrm{~mm}$ à celle des tegulae, soit une réduction de 17,2\%. Le constat est le même pour la canalisation 3703 (12,3 \%), l'US 1203 (11,2 \%), l'US 1397 
(16,5\%) et l'US 1288 (10,1\%). Aux «Cléons » (26 individus), la tendance est confirmée, les imbrices étant en moyenne plus courtes de 13,4\%.

Le modèle de pose retenu en général pour une toiture romaine est celui d'une imbrex venant en butée contre chaque tegula amont (fig. 5), ce qui induit un nombre d'imbrices identique à celui des tegulae.

Cependant, dans ce cas, le recouvrement entre imbrices est très faible. À Angers, pour la toiture 3206, ce recouvrement n'est plus que de $35 \mathrm{~mm}$ (contre $87 \mathrm{~mm}$ pour les tegulae) et n'est pas accompagné de mortier d'étanchéité. Pour une toiture actuelle de type « tuile canal », le recouvrement conseillé est compris entre 14 et $17 \mathrm{~cm}$ et ce, pour des pentes supérieures.

On peut poser l'hypothèse que le nombre d'imbrices disposé sur une rangée de tegulae était supérieur au nombre de celles-ci. Cependant, cette mise en œuvre n'est que peu satisfaisante en terme d'étanchéité, puisqu'elle occasionne un décollement important des imbrices sur les tegulae. La question reste ouverte à ce stade de l'étude.

\section{L'usage de mortier pour la pose des couvre-joints}

Cet usage est attesté archéologiquement sur de nombreux sites à Angers «SaintLouis ", à Angers « Marengo » (Pithon, 2012), à Haute-Goulaine "Les Cléons ", à Piriacsur-Mer «ZAC du Pladreau» (Hervé, 2007), à Jard-sur-Mer «Le Grand Essart» (Nauleau, 2009b) et au Langon « Rue de l'Industrie » (Hervé, 2008).

28 La section transversale des boudins de mortier est caractéristique, avec une surface supérieure convexe correspondant à la surface interne de l'imbrex et une surface inférieure portant la marque de rebords de deux tegulae (fig. 6). La pose avec mortier permet d'assurer une meilleure étanchéité et de réduire le glissement des files d'imbrices.

Figure 6 : Mortier résiduel sous imbrex, Tc 884 Angers « Saint-Louis ». Figure 6 : Residual mortar under imbrex, Tc 884 Angers "Saint-Louis"

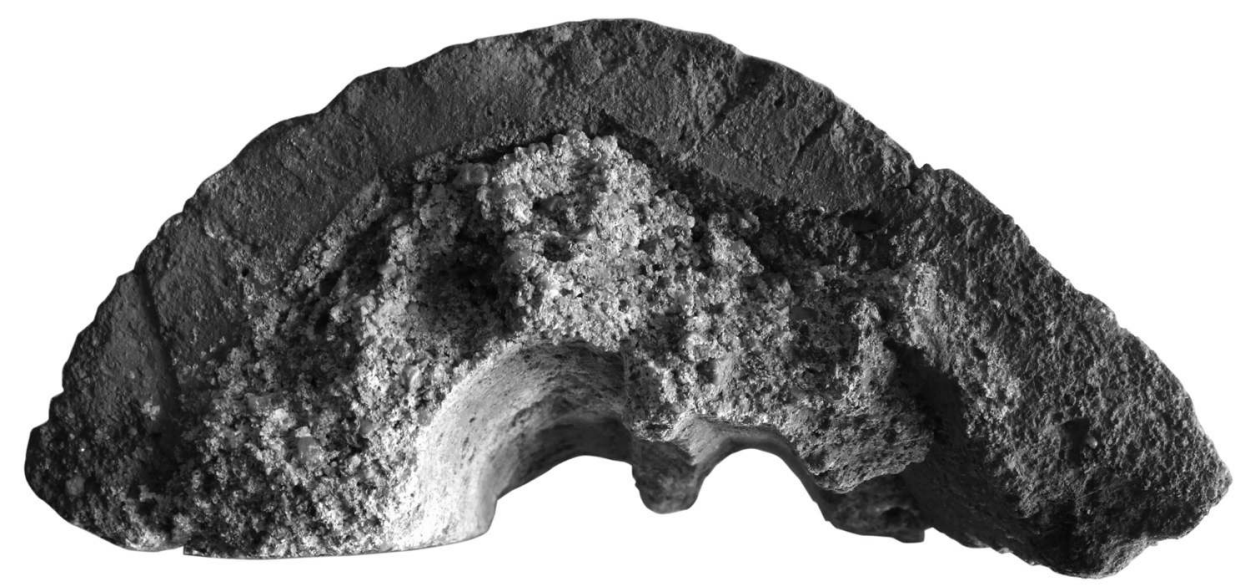

Cliché J.-F. Nauleau, Inrap.

29 Cet usage est probablement loin d'être systématique et il est absent sur des toitures effondrées bien documentées archéologiquement (toiture effondrée 3206 de la domus 
d'Angers « Saint-Louis », début/milieu III siècle apr. J.-C.). Il est possible que ce mortier n'ait été utilisé qu'en partie basse de toiture, pour la première imbrex à l'égout du toit.

\section{Les tuiles de faîtage et tuiles d'arêtiers}

Les tuiles de faitage attestées sont archéologiquement rares. Seuls sept cas seront présentés ici (fig.7). Deux ne présentent aucune morphologie spécifique et sont simplement des imbrices de grande taille. Elles proviennent du site des "Cléons », et leur morphologie est parfaitement identique aux imbrices classiques: seule leur longueur est exceptionnelle, avec des valeurs de 440 et $442 \mathrm{~mm}$. Leur grande largeur (partie aval) est de 215 et $210 \mathrm{~mm}$.

Figure 7 : Les tuiles de faîtage.

Figure 7 : Roofing tile.

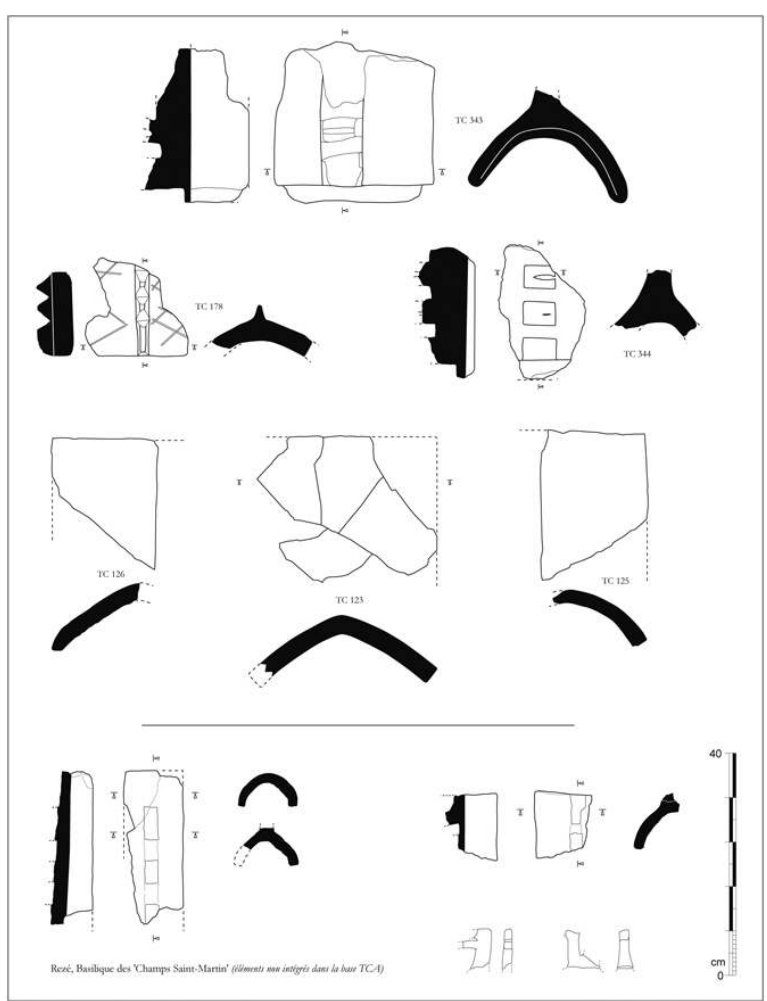

Tc 343, 344 : Haute-Goulaine « Les Cléons »; Tc 123, 125, 126 : Le Langon « Rue de l'Industrie » ; Tc 178 : Beaufort-en-Vallée " Chemin des Airaults ».

Tc 343, 344: Haute-Goulaine "Les Cléons"; Tc 123, 125, 126: Le Langon "Rue de I'Industrie"; Tc 778 Beaufort-en-Vallée "Chemin des Airaults".

Au Langon « Rue de l'Industrie », au moins deux exemplaires d'un type bien différent ont été mis au jour. Il s'agit de tuiles de type imbrex mais avec un profil dièdre de type « corinthien ». Elles sont très larges, une des tuiles affichant $340 \mathrm{~mm}$. La longueur n'est pas connue mais devait être également très importante, aux alentours de $500 \mathrm{~mm}$.

Trois autres éléments remarquables sont en revanche munis d'un décor, deux sur le site des "Cléons » et un à Beaufort-en-Vallée «Le chemin des Airaults» (Tourneur, 2006). L'exemplaire de Beaufort-en-Vallée présente une ornementation intacte. Il s'agit d'une tuile dont la crête festonnée haute d'environ $30 \mathrm{~mm}$ présente des découpes triangulaires. Les flancs sont incisés par des lignes peu marquées formant un motif 
losangé. Notons que ce type de décor à crête festonnée est proche de ceux rencontrés au Moyen Âge (Poole, 2007).

Dans le cas des "Cléons ", il s'agit de deux tuiles très robustes dont les épaisseurs varient de 35 à $40 \mathrm{~mm}$. Une des deux tuiles présente une largeur conservée de $290 \mathrm{~mm}$. Les deux exemplaires sont munis d'un dispositif d'emboîtement sous la forme d'une languette longue d'environ $30 \mathrm{~mm}$. Le décor est constitué d'une crête découpée, dont seule subsiste la base (fig. 8). Les perforations visibles au centre des parties qui s'élevaient en motifs ne suggèrent pas obligatoirement qu'il s'agissait de motifs échancrés. Des percements destinés à favoriser le séchage et la cuisson ont été observés sur les exemplaires médiévaux (ibid.).

Figure 8 : Tuile faîtière de Haute-Goulaine "Les Cléons », Tc 343.

Figure 8 : Roofing tile from Haute-Goulaine "Les Cléons", Tc 343

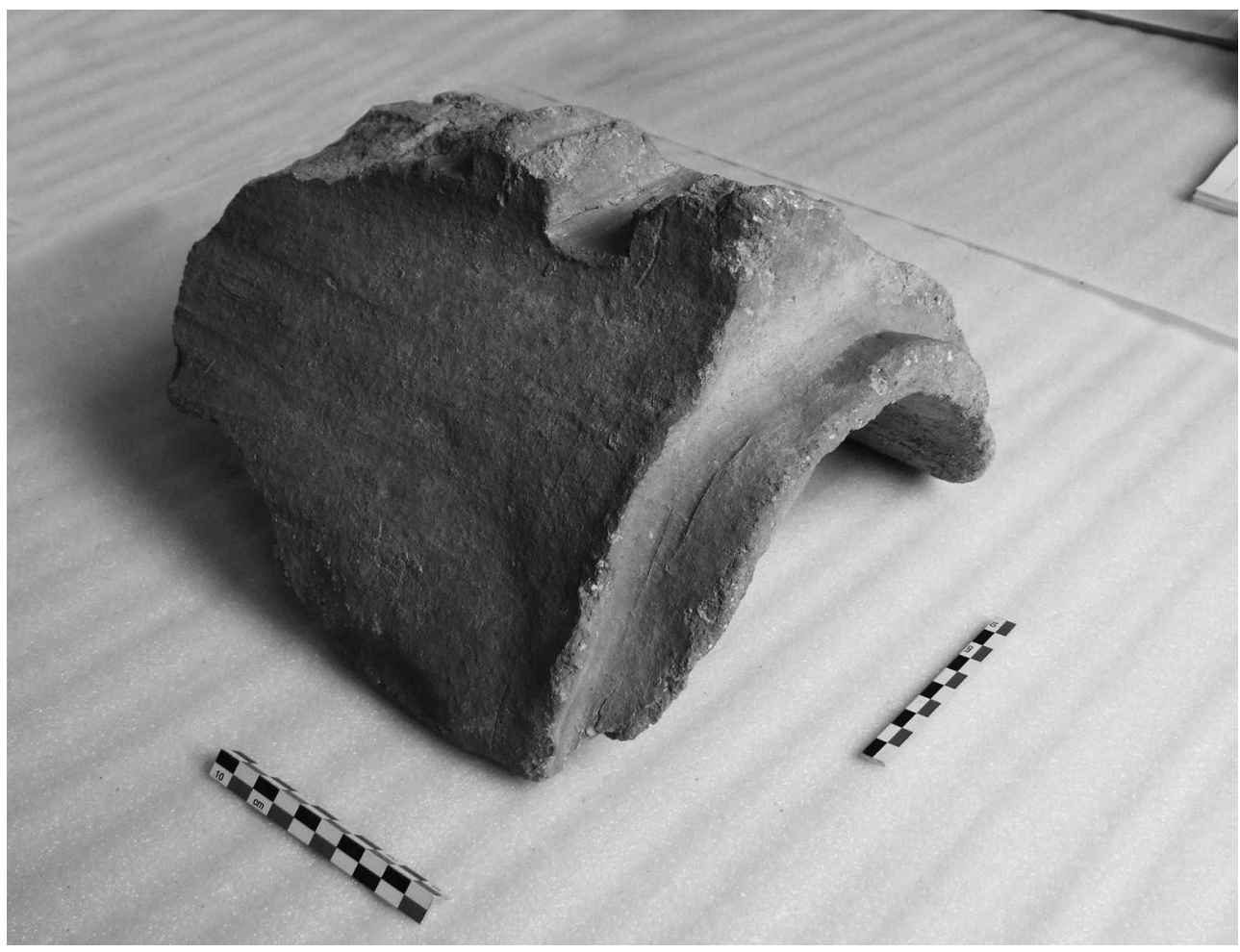

Cliché J.-F. Nauleau, Inrap.

Cet exemplaire n'est pas sans évoquer les fragments découverts dans la basilique des Champs Saint-Martin à Rezé (début $\mathrm{VI}^{\mathrm{e}}$ siècle apr. J.-C., Pirault, 2009). Un ensemble remarquable de petites tuiles de faitage a été mis au jour (deux largeurs conservées de 110 et $150 \mathrm{~mm}$ ) et arborent une crête à motifs complexes échancrés dont aucun ne nous est parvenu intact.

Ces tuiles étaient, d'après l'auteur de la fouille, probablement mises en œuvre sur la couverture d'un ciborium situé dans le chœur, qui aurait reproduit à petite échelle une structure architecturale plus vaste (communication orale, L. Pirault). Ce type de modèle réduit d'édifices n'est pas sans évoquer les maquettes architecturales en terre cuite rencontrées dans le monde romain : citons localement celles mises au jour récemment à Rennes (Maligorne et al., 2010). 
Dans un cadre géographique plus large, on peut citer les tuiles faîtières en provenance de Drevant dans le Cher (Bailly, 1981) à motif ajouré complexe. Dans le Loir-et-Cher, à Moncelon, des tuiles très atypiques ont été découvertes : il s'agit d'ornements en forme de dauphins, sans doute destinés à décorer une faîtière d'environ $0,45 \mathrm{~m}$ de longueur. Le contexte architectural des découvertes est mal connu (Ferdière, 1988).

\section{Les antéfixes}

37 Ce sont des matériaux emblématiques de l'archéologie gallo-romaine mais ils sont en réalité peu nombreux à avoir été mis au jour dans la région. Ils sont destinés à décorer les extrémités des lignes d'imbrices à l'égout du toit.

Les antéfixes sont le plus fréquemment ornées de motifs stylisés, de palmettes, de crosses, mais peuvent aussi être figuratives (satyres, par exemple).

Seuls deux exemplaires sont enregistrés dans la base, provenant de la fouille récente d'Angers "Saint-Louis", mais deux autres sont connus également à Angers (non intégrés dans la base), l'un en provenance des fouilles de la "Terrasse du logis royal » au château et l'autre de la " Rue Chaussée Saint-Pierre » (Mortreau dans Pithon, 2011).

Trois de ces antéfixes semblent appartenir à un même type. La partie antérieure est ornée de cordons concentriques larges et hauts d'environ $5 \mathrm{~mm}$, dessinant deux volutes à enroulement interne, encadrant dans deux des trois cas un visage surmonté d'un arbre ou d'une colonne.

41 Le second type est une antéfixe de format trapézoïdal, avec une largeur basse d'environ $180 \mathrm{~mm}$ (fig. 9). Sur la face antérieure est figuré un personnage joufflu au nez busqué et à moustache, dont les incisions sur les joues représentent probablement une barbe (un Silène ?). 
Figure 9 : Une antéfixe (Tc 978, Angers « Saint-Louis »). Figure 9 : Antefix (Tc 978, Angers "Saint-Louis").

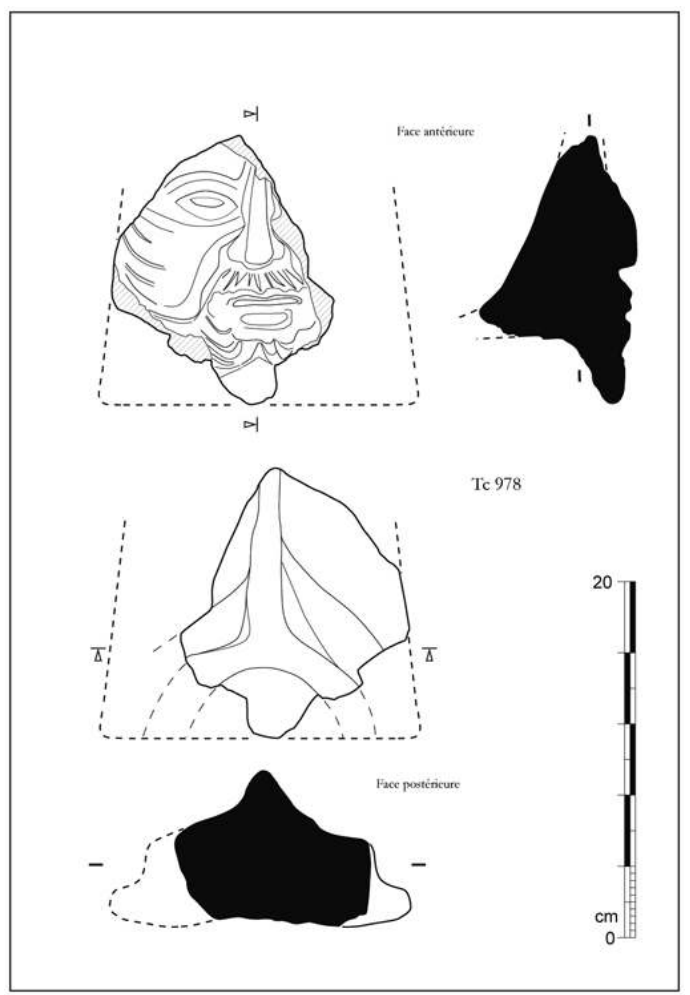

En face postérieure sont parfaitement visibles la naissance de sa partie «imbrex » et le dispositif de renfort permettant de conforter la fixation entre cette partie horizontale et la partie antérieure verticale. Il s'agit d'un large renfort oblique venant s'insérer au dos de cette partie antérieure. Ils sont souvent figurés dans les reconstitutions d'édifices, mais ils devaient être très rares sur les toitures romaines, limités à certains bâtiments prestigieux, publics ou privés.

\section{Tuiles en écaille}

Deux exemplaires de tuiles très atypiques régionalement ont été découverts aux "Cléons » (fig. 10). Il s'agit d'éléments de forme ovale mais tronqués de chaque côté en partie haute, longs de $330 \mathrm{~mm}$ et large d'environ $200 \mathrm{~mm}$. Leur partie haute est marquée par une perforation réalisée avant cuisson. 
Figure 10 : Les tuiles en écaille, Haute-Goulaine "Les Cléons ». Figure 10 : Tiles in scale pattern, Haute-Goulaine "Les Cléons".

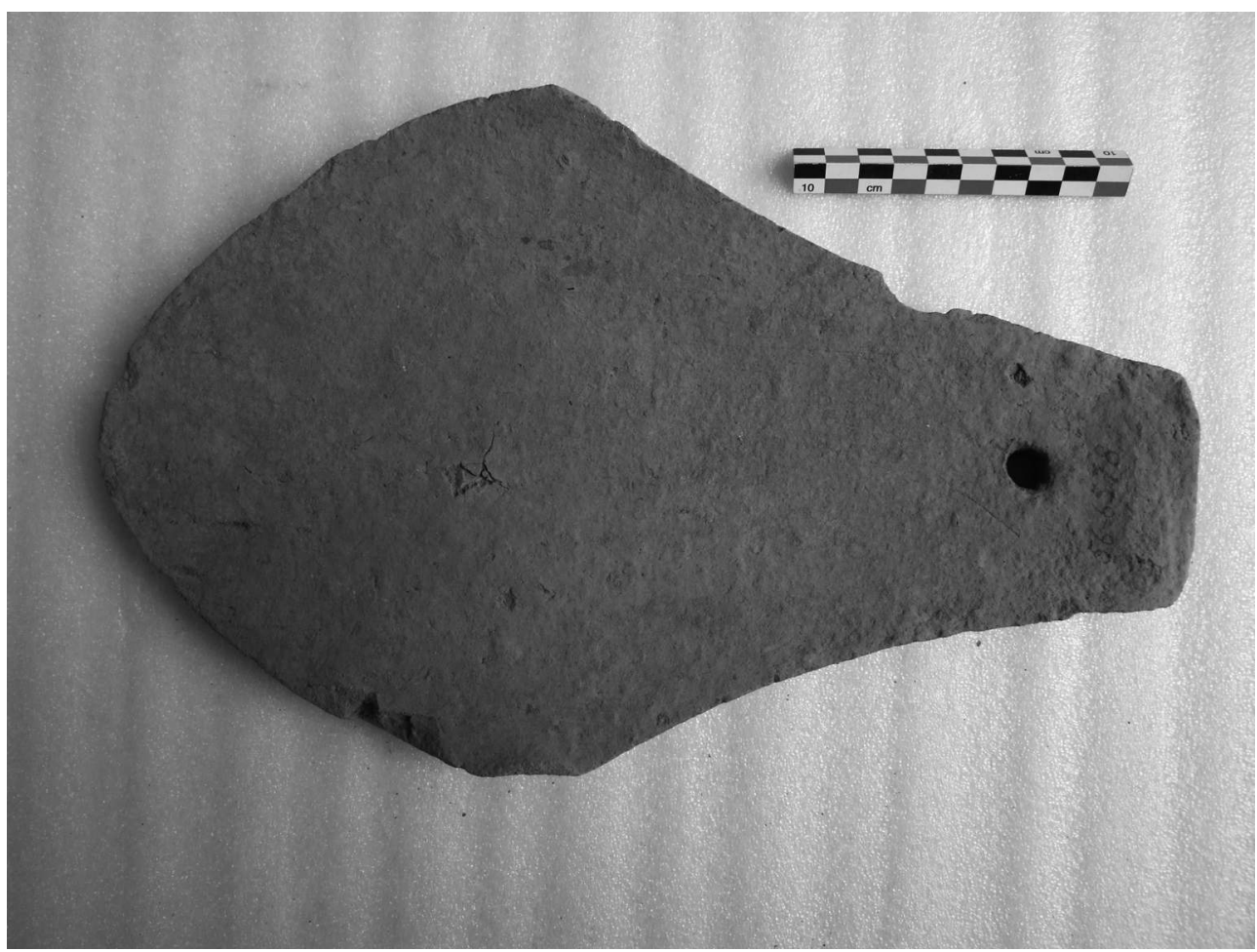

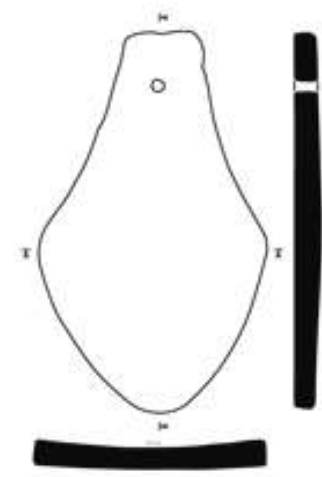

I. 35
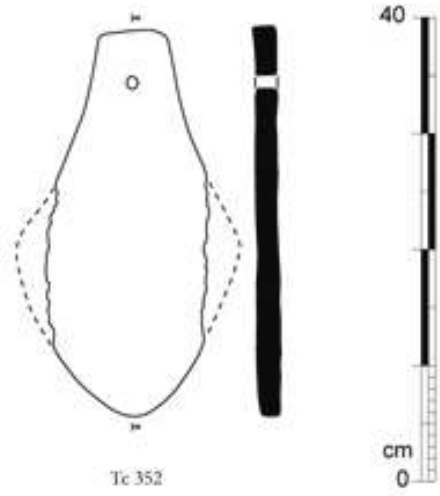

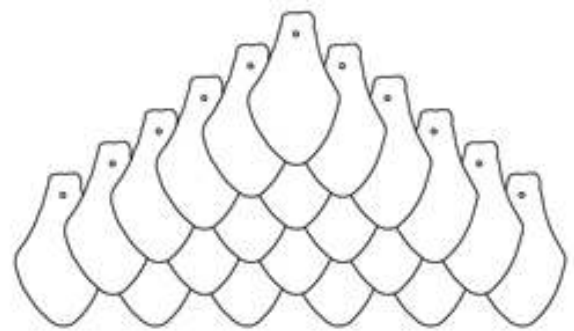

Leur mise en œuvre sur une toiture paraît réalisable, avec un mode de pose de type ardoise, à petit pureau ${ }^{5}$ et pose alternée. Elles évoqueraient alors les tuiles-écailles modernes et contemporaines rencontrées dans le Dauphinois (Chauvet, 1996, p. 88). Cependant, même avec un recouvrement important, l'étanchéité semble délicate à assurer, compte tenu de la morphologie de la partie amont. 

(Rezé, un mur de la domus "Terrain Peigné»). On rencontre la brique dans bien d'autres édifices, des canalisations et des bassins (« quartier Saint-Lupien » à Rezé), des fours domestiques (domus du «Terrain Peigné»), ainsi que des soles de séchoir (Rezé, « quartier Saint-Lupien », FR 1125), par exemple.

51 Ces briques rectangulaires présentent différents formats dont certains semblent très répandus localement et d'autres plus spécifiques (fig. 11). Le format des briques dites «lydienne", de format $1 \times 1,5$ pied (Goulpeau, 1988), soit $445 \times 297 \mathrm{~mm}$, et traditionnellement associé à ces briques rectangulaires dans le monde romain, est en réalité très peu courant dans l'aire d'étude. Un seul exemplaire a été découvert à Angers « Saint-Louis », dont le format est 443 x $295 \mathrm{~mm}$. 
Figure 11 : Format des briques quadrangulaires rencontrées (hors mesures sur élévations). Figure 11 : Formats of the quadrangular bricks (excluding measurements made on the elevations)

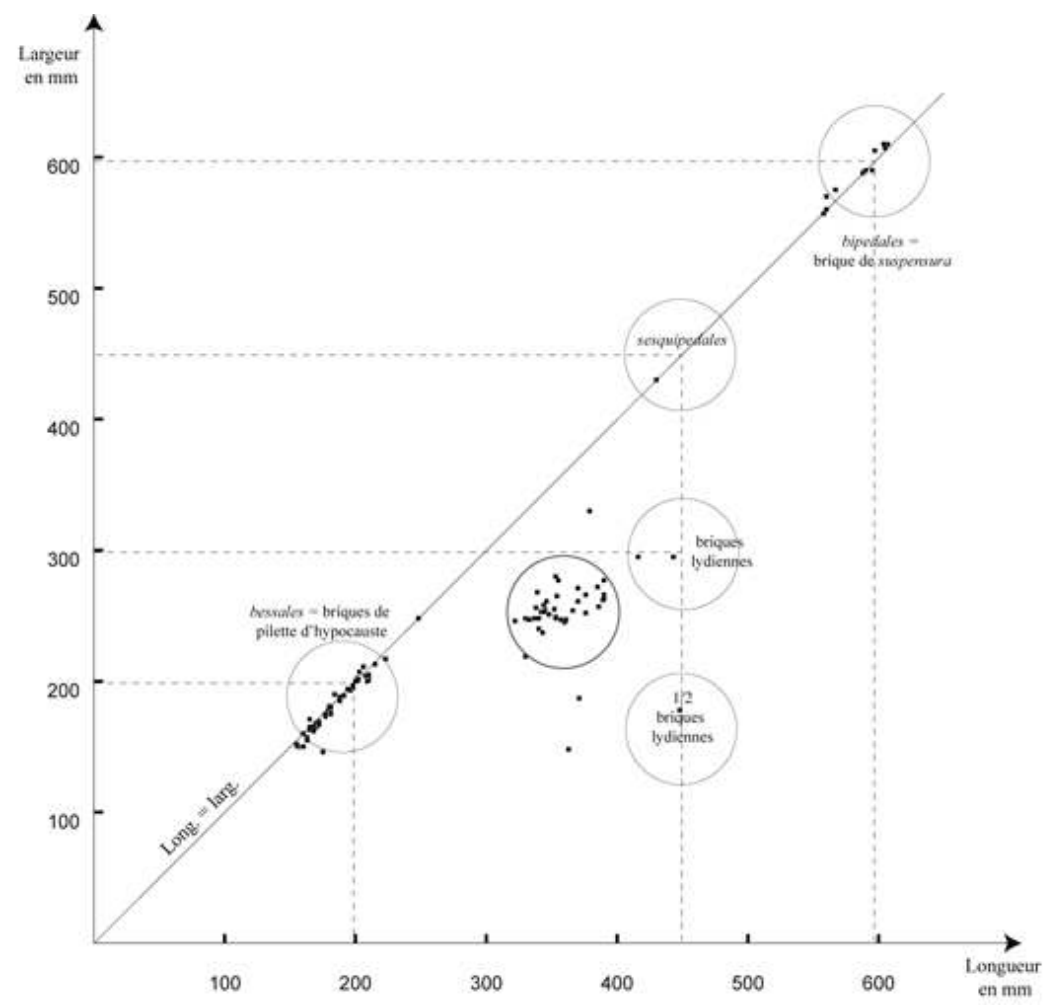

Le module nettement dominant est plus petit : $340 / 350$ x $250 \mathrm{~mm}$. Il a été observé dans le « quartier Saint-Lupien » à Rezé, dans toutes les constructions qui se développent à partir de la fin $d u I^{\text {er }}$ ou au début du $\mathrm{II}^{\mathrm{e}}$ siècle apr. J.-C. Près de 160 mesures ont été effectuées dans ces maçonneries ${ }^{7}$, les moyennes obtenues étant de 347 x $249 \mathrm{~mm}$. Dans le même quartier, le four FR 1125 a livré treize exemplaires de ce format (338 x $249 \mathrm{~mm}$ en moyenne) et onze autres exemplaires proviennent des "Cléons ", où leur moyenne est de 342 x $252 \mathrm{~mm}$. C'est encore ce format qui est abondamment mis en œuvre dans des canalisations et des murs de la domus du «Terrain Peigné » vers la fin du $\mathrm{I}^{\mathrm{er}}$ siècle apr. J.-C. Enfin, dans la villa de Piriac-sur-Mer «ZAC du Pladreau », les ouvertures des praefurnia des thermes employaient des briques de même format. Les épaisseurs de ces briques sont faibles, en moyenne de $37 \mathrm{~mm}$ pour le corpus de Rezé.

D'autres formats moins fréquents ont été identifiés. Parmi ces briques, on peut citer des formats 380/390 x 260/270 mm rencontrés en cinq exemplaires, 385 x 272 mm (Angers "Saint-Louis »), 380 x $260 \mathrm{~mm}$ (deux exemplaires à Rezé), $390 \times 264 \mathrm{~mm}$ (deux exemplaires aux «Cléons »). Citons encore un format 380 x $330 \mathrm{~mm}$, en provenance des «Cléons» (quatre exemplaires) et un format 416 x $295 \mathrm{~mm}$ (un exemplaire, Angers « Saint-Louis »). À Gennes-sur-Loire (49), les modules rencontrés au sein d'un caniveau appartenant à l'édifice de spectacle sont 385 x $315 \mathrm{~mm}$ et 385 x $285 \mathrm{~mm}$ (Bouvet, 1987).

Notons enfin des briques de demi-format, probablement destinées à être mises en œuvre dans des chaînages d'angle ou des piédroits de baies pour assurer la continuité de la pose à joints croisés.

On possède peu d'éléments de comparaison en ce qui concerne le format des briques de maçonnerie. On peut noter que le format prépondérant ici est celui qui domine aussi largement au sein du castrum de Jublains (Le Bohec, 1983). On ne le rencontre pas dans 
le Sud de la Gaule (De Filippo, 2004), ni dans les thermes de Cluny à Lutèce (Adam, 2008, p. 160), ni dans la domus du «Bas de Vieux» à Vieux (Calvados) (Vipard, 1999). On possède à l'heure actuelle trop peu de données pour tirer des conclusions à partir de ces observations, mais notons que ce format $340 / 345 \times 250 \mathrm{~mm}$ semble largement dominant dans l'aire d'étude et n'est que peu ou pas décrit ailleurs.

Dans la plupart des cas, en face inférieure, une encoche (fig. 12) est réalisée après un certain temps de séchage, que l'on nomme en général poignée de préhension. Le format $340 \times 250 \mathrm{~mm}$ est celui qui possède le plus fort taux de poignées ( 28 briques sur 31 ) et elles paraissent nettement plus rares sur les autres formats de brique. Cette poignée est utile à la manutention des briques sur le lieu de production aussi bien que sur le chantier où elles seront mises en œuvre. La présence de cette poignée peut aider à discriminer les briques peu épaisses de tegulae fracturées robustes.

Figure 12: Poignée de préhension en face inférieure d'une brique, Haute-Goulaine "Les Cléons ". Figure 12 : Gripping element on the lower face of a brick, Haute-Goulaine "Les Cléons".

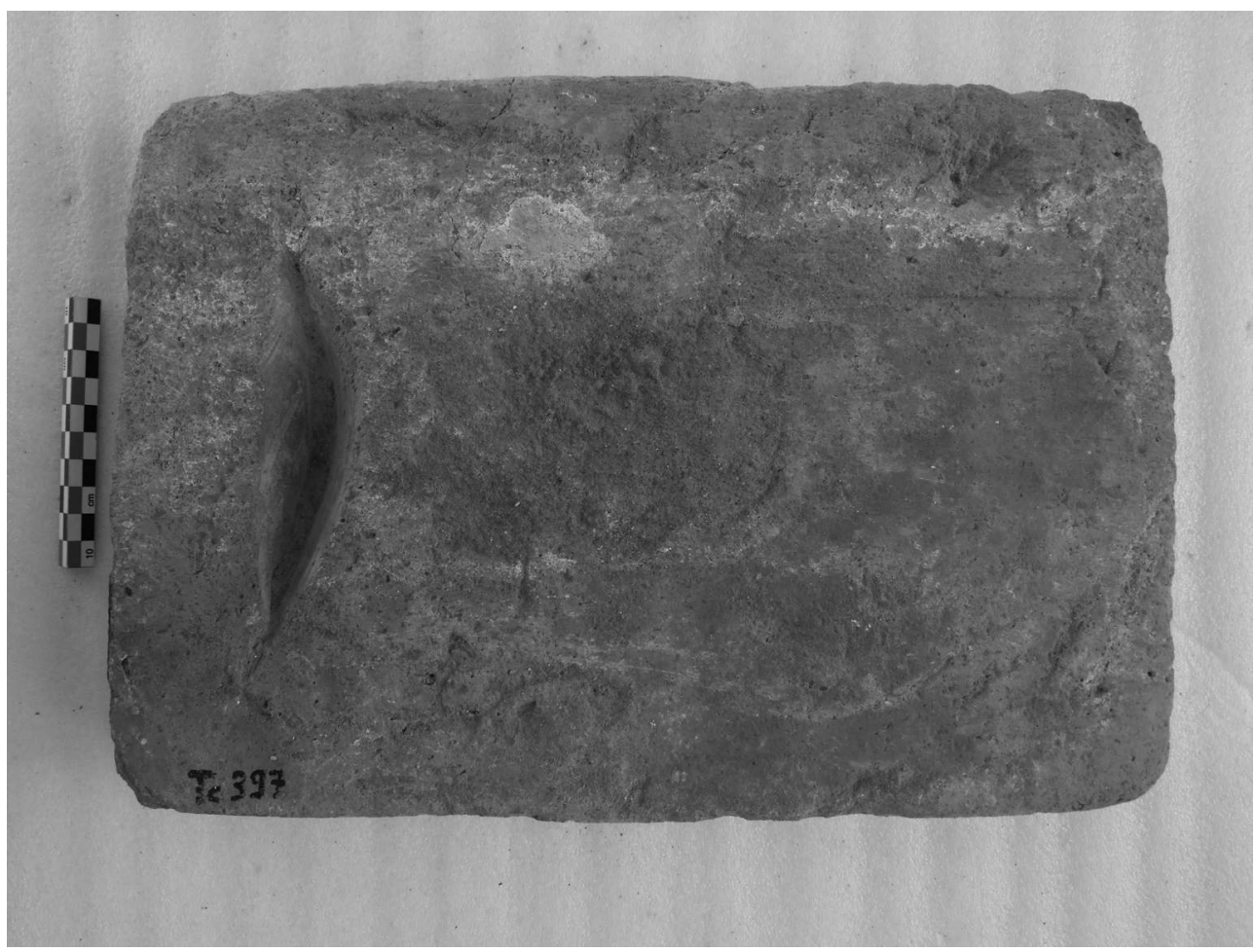

Cliché J.-F. Nauleau, Inrap.

Les données chronologiques se rapportant à l'usage de la brique dans le monde romain sont peu précises dans la mesure où l'on se heurte à la difficulté de datation des vestiges bâtis. Globalement, on prétend que l'apparition du mur à chaînage de briques intervient vers la fin du $\mathrm{I}^{\mathrm{er}}$ - début du $\mathrm{II}^{\mathrm{e}}$ siècle apr. J.-C. (Hofmann, 1975 citant Albert Grenier, et Adam, 2008, p. 154). Cependant, il faut nuancer ce schéma global qui semble souffrir d'exceptions notables (De Filippo, 2004).

Localement, on possède quelques données permettant de préciser l'histoire de l'usage de la brique. En ce qui concerne leur date d'apparition, mentionnons l'existence d'un raté de cuisson remployé en maçonnerie dans un pilier de support de sole du four de tuilier FR 7030 à Rezé «quartier Saint-Lupien ». Ce four est scellé par un niveau de 
démolition qui place son usage dans les toutes premières décennies du $\mathrm{i}^{\mathrm{er}}$ siècle de notre ère. La brique mentionnée, archéologiquement complète, présente un format de 380 x $290 \mathrm{~mm}$. À Angers "Saint-Louis ", les constatations sont les mêmes : la brique (de format inconnu) est présente dans des niveaux augustéens bien datés (US 2397, 2426 et 2441). D'après ces différentes observations, les briques rectangulaires seraient apparues tôt, en tout cas approximativement en même temps que les matériaux de couverture. À Rezé, dans le "quartier Saint-Lupien", l'apparition de l'opus mixtum semble se faire vers la fin du $\mathrm{I}^{\mathrm{er}}$ ou au début du $\mathrm{II}^{\mathrm{e}}$ siècle apr. J.-C., en concordance avec les propositions d'A. Grenier. Quel était alors l'usage réservé aux briques produites dès le début et durant tout le $\mathrm{I}^{\mathrm{er}}$ siècle de notre ère ? Leur usage en soles de fours, canalisations, voire mur-bahut, peut être avancé, mais aucun témoignage archéologique ne permettant d'étayer ces hypothèses, la question reste donc ouverte.

Par ailleurs, on ne possède pas suffisamment de données pour mettre en évidence une évolution éventuelle du format de ces briques à maçonner. Dans le Sud de la Gaule, on note localement une évolution à la baisse, comparable à celle des tegulae. À Toulouse, par exemple, un format de $370 \times 250 \mathrm{~mm}$ succède à un format "lydien » de 450 x 300 mm (De Filippo, 2004).

À Rezé, le format de 380 x $290 \mathrm{~mm}$ du début du $\mathrm{I}^{\mathrm{er}}$ siècle apr. J.-C. semble évoluer vers celui plus réduit de $340 / 345 \times 250 \mathrm{~mm}$ à la fin du $\mathrm{I}^{\mathrm{er}}$ ou au début du $\mathrm{II}^{\mathrm{e}}$ siècle apr. J.-C. ${ }^{8}$. Cependant, les observations sur les briques précoces sont trop ponctuelles et les données chronologiques ne sont pas les seules à devoir être prises en compte, différents formats pouvant être associés simultanément pour différents usages.

\section{Les briques de colonne}

61 Ces briques sont maçonnées à joints croisés dans le but d'élever des fûts de colonnes (fig. 13). Elles sont ensuite systématiquement enduites de façon à imiter des fûts monolithes, ce qui explique le peu de soin réservé en général à leur façonnage. Un ensemble maçonné d'une dizaine d'individus a été mis au jour aux «Cléons », formant une colonne de $280 \mathrm{~mm}$ de diamètre (sans les enduits de surface). 
Figure 13 : Lot de briques quart-de-rond, Haute-Goulaine "Les Cléons ». Figure 13 : Quarter shaped bricks, Haute-Goulaine "Les Cléons".

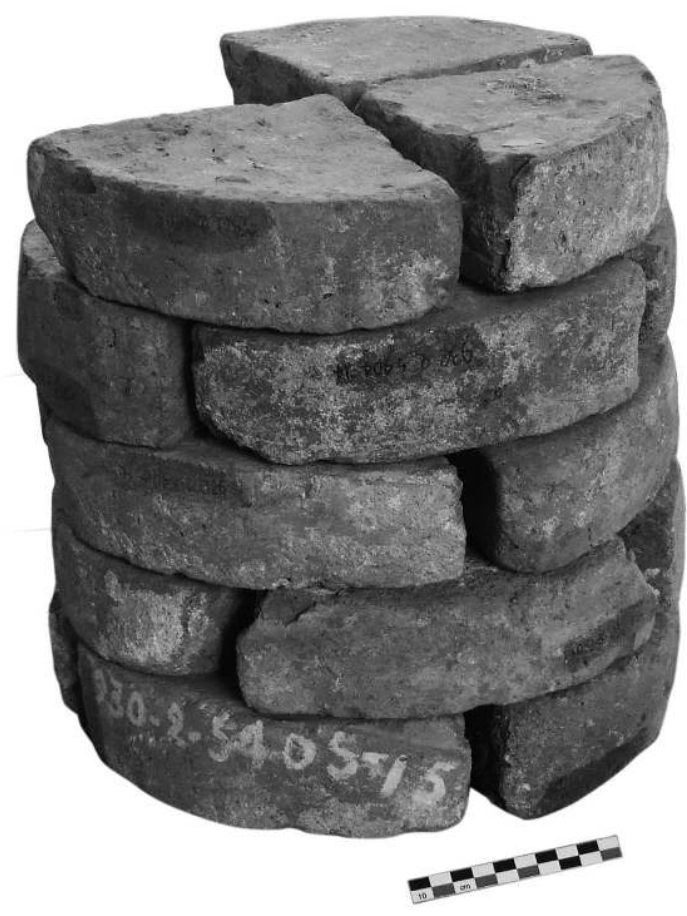

Cliché J.-F. Nauleau, Inrap.

Cent treize individus ont été recensés sur seulement cinq sites (fig. 14) dont quatre de probables villae. Le site des "Cléons " à lui seul a fourni soixante-douze exemplaires", celui de Piriac-sur-Mer, neuf exemplaires et enfin celui de Jard-sur-Mer, six exemplaires. Un seul site urbain, celui de Rezé, a livré treize exemplaires mis en œuvre pour une canalisation de la domus du "Terrain Peigné ». Dix exemplaires conservés au musée Dobrée sont de provenance inconnue.

Figure 14 : Histogramme cumulé des diamètres des briques de colonne rencontrées. Figure 14 : Bar chart of the diameters of the column bricks

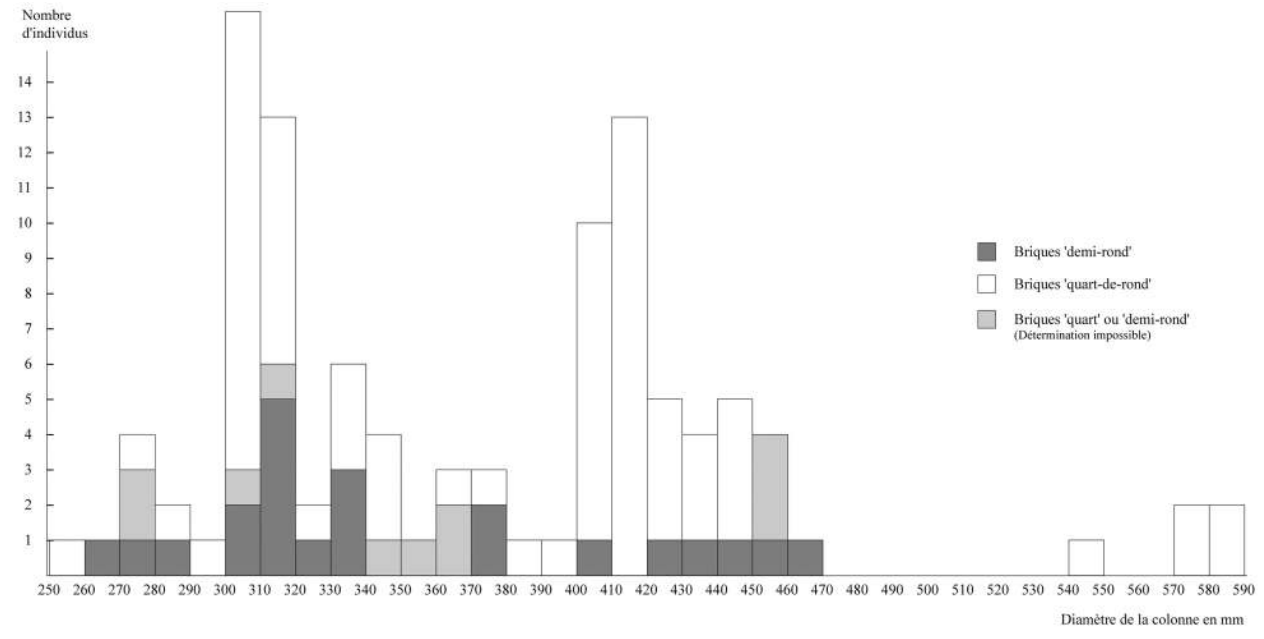



(73 exemplaires avérés contre 22). Les deux types se retrouvent dans tout l'éventail des diamètres rencontrés. Ces briques forment rarement un quart ou demi-cercle complet du diamètre concerné, cette marge correspondant à la largeur du joint de mortier qui est ensuite réalisé. $590 \mathrm{~mm}$, mais l'histogramme de fréquence fait apparaître des tendances. Les diamètres les plus répandus sont de $300 / 320$ et $400 / 420 \mathrm{~mm}$. Le lot étudié est cependant déséquilibré puisque représenté par plus de $60 \%$ de briques du seul site des «Cléons ». N'oublions pas que ces briques sont systématiquement enduites, l'épaisseur de l'enduit pouvant ajouter entre 50 et $100 \mathrm{~mm}$ au diamètre total de la colonne. Deux exemplaires découverts à Piriac-sur-Mer conservent les différentes couches d'enduit (Tc 112 et Tc 115).

66 En incluant l'épaisseur de l'enduit, les deux diamètres de colonne les plus rencontrés sont donc $380 / 400$ et $480 / 500 \mathrm{~mm}$. Ces valeurs devront être confrontées aux diamètres habituellement rencontrés pour les fûts de colonne monolithes ou à tambours. Ces briques pouvaient aussi être utilisées pour édifier des colonnes adossées non fonctionnelles (demi-colonnes).

67 Ces briques sont finalement assez peu répandues et se rencontrent surtout en contexte de villa. À titre d'exemple, la vaste fouille récente d'Angers « Saint-Louis », portant sur un îlot de la ville antique de Juliomagus et incluant une vaste domus, n'a pas livré de matériau de ce type.

68 Ces briques sont-elles un indicateur de qualité architecturale? La question est probablement complexe car les deux types de colonne devaient pouvoir cohabiter et, par ailleurs, l'usage de piliers en bois n'est pas à exclure.

\section{Quelques briques atypiques}

69 Des petites briques au format singulier ont été trouvées dans les niveaux d'abandon du four de tuilier des Clouzeaux en Vendée (Séris, 2008). Il s'agit de briques triangulaires d'une quinzaine de centimètres de côté, dont l'épaisseur décroit sur l'un des côtés (fig. 15). 
Figure 15 : Les briques triangulaires (Les Clouzeaux « La Grosse Pierre »). Figure 15 : Triangular shaped bricks (Les Clouzeaux "La Grosse Pierre").

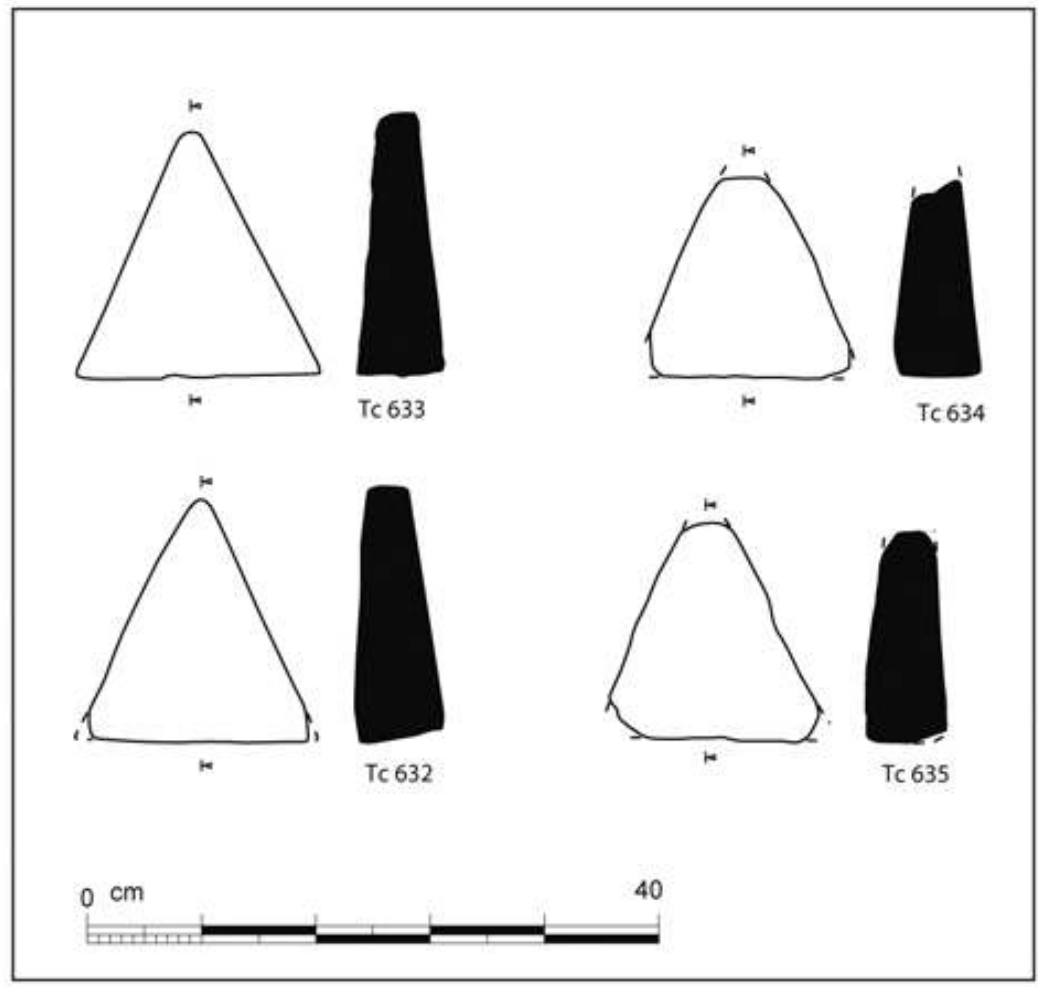

70 La morphologie de ces briques évoque celle des moellons utilisés dans l'opus vittatum avec un parement quadrangulaire et une racine se terminant en pointe.

71 Les briques triangulaires sont connues dans le monde romain: elles résultent en général de la taille après cuisson de briques carrées ou quadrangulaires (De Filippo, 2004, p. 104). Le plus grand côté du triangle est alors placé en parement. L'intérêt d'une telle pratique est mal connu : peut-être permet-elle une économie de matériaux. Ce mode d'emploi de la brique n'est en tout cas pas connu en Gaule.

Les briques présentées ici ne semblent d'ailleurs pas entrer dans cette catégorie car elles ne sont pas taillées après cuisson et sont nettement plus épaisses $(60 \mathrm{~mm})$.

Des briques de ce type pourraient être mises en œuvre localement dans la muraille du Bas-Empire de Nantes, en lieu et place des moellons de granite habituellement rencontrés (observation "École Saint-Pierre»). Une étude plus spécifique mériterait d'être menée sur cette question.

\section{Les matériaux spécifiques au chauffage par le sol}

74 Ces matériaux sont bien connus de la plupart des archéologues. Il s'agit principalement de briques composant les pilae (pilettes) d'hypocauste et de briques constituant la suspensura, ainsi que des tubulures murales permettant la circulation de l'air chaud. On ne s'étendra donc pas sur ces trois types de matériaux, mais on insistera en revanche sur d'autres matériaux moins fréquents et probablement moins bien identifiés sur les sites. 


\section{Les briques de pilettes} gallo-romain. Il s'agit de briques de format carré10 dont la largeur avoisine les 20 centimètres de côté ( $2 / 3$ de pied romain), appelées «bessales ». Cependant, des différences sont notables selon les sites et cette valeur ne représente qu'une moyenne. Aux « Cléons », les valeurs s'échelonnent de 150 à $215 \mathrm{~mm}$.

Sur ce site même, des familles ont pu être identifiées grâce au type de pâte et aux stigmates de façonnage (fig. 16). Des variations considérables d'épaisseur ont été constatées, de $38 \mathrm{~mm}$ jusqu'à $88 \mathrm{~mm}$, les matériaux les plus épais n'étant d'ailleurs pas les plus grands. Ces valeurs hautes sont inattendues dans la mesure où elles sont supérieures à celles des bipedales de suspensura, briques carrées de $60 \mathrm{~cm}$ de côté. Elles n'ont été constatées que sur ce site : ailleurs, les valeurs habituellement rencontrées sont proches de $40 / 45 \mathrm{~mm}$.

Figure 16 : Les briques « bessales » de Haute-Goulaine " Les Cléons ». Figure 16 : "Bessal" bricks from de Haute-Goulaine "Les Cléons".

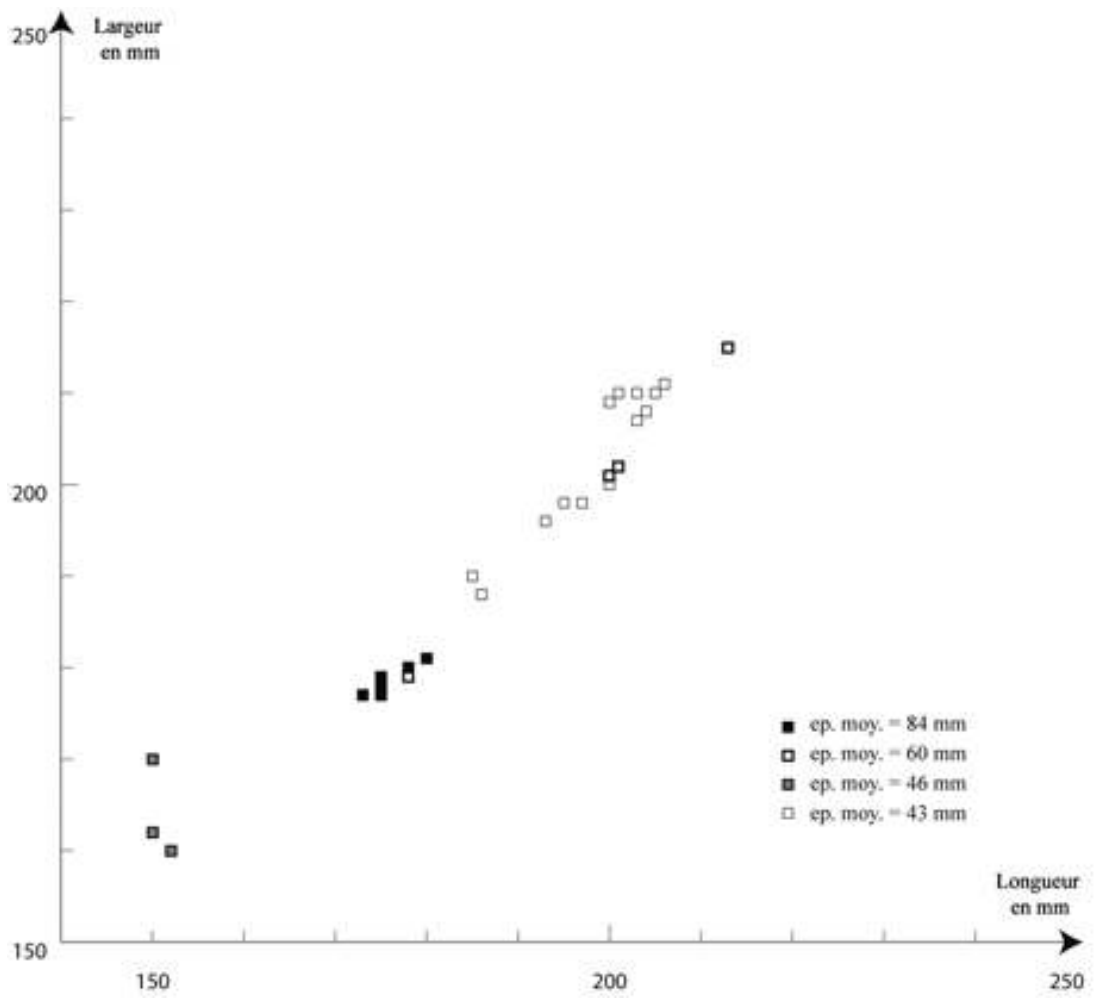

\section{Les briques de suspensura}

Ces briques ne sont pratiquement jamais trouvées intactes. Il s'agit de briques de format carré d'environ $60 \mathrm{~cm}$ de côté (briques bipedales de deux pieds romains, soit exactement $594 \mathrm{~mm}$ ), destinées à être posées sur les pilettes et supportant les couches de forme de la suspensura. Dix exemplaires remployés en carreaux de sol ont été découverts aux "Cléons ». Ils sont tous très proches des deux pieds romains de côté (590 $\mathrm{mm}$ en moyenne) et assez peu épais ( $55 \mathrm{~mm}$ en moyenne), compte tenu de leur dimension. 


\section{Les cloisons creuses} des «Cléons » (fig. 17). Les tailles sont très variables : l'effectif des éléments étudiés étant faible, il est difficile de dégager des tendances. Notons des petits formats de $345 \times 130 \times 70 \mathrm{~mm}$ et $325 \times 180 \times 135 \mathrm{~mm}$ et un grand format de $385 \times 220 \times 160$. Les tubuli les plus grosses pourraient constituer les collecteurs situés dans les parties hautes des pièces chauffées et s'apparenteraient davantage à nos boisseaux de cheminée actuels. Les formats plus réduits, les plus fréquents, semblent être ceux qui sont utilisés en grand nombre pour constituer des cloisons chauffées.

Figure 17 : Les tubuli. Figure 17 : Tubuli.

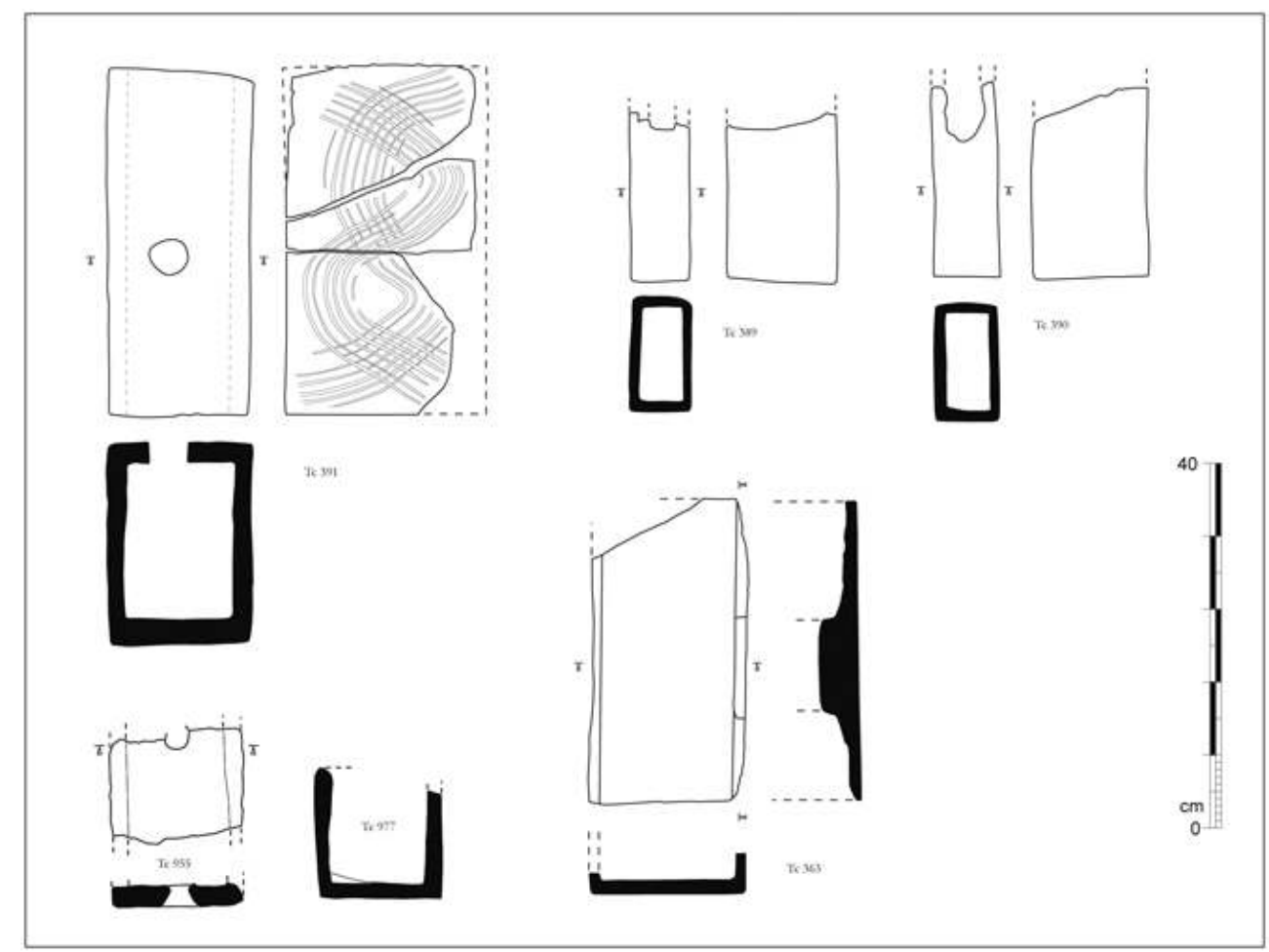

Tc 363, 389, 390, 391 : Haute-Goulaine «Les Cléons »; Tc 955, 977 : Angers « Saint-Louis ». Tc 363, 389, 390, 397 : Haute-Goulaine "Les Cléons"; Tc 955, 977 : Angers "Saint-Louis".

Notons qu'on trouve aussi des tubuli en quantité moindre dans le cas d'hypocauste simple, alors utilisées en conduit d'évacuation des gaz, situé à l'opposé du praefurnium. C'est le cas pour le site d'Angers «Saint-Louis », dans la salle sur hypocauste d'une domus où les tubulures sont de section carrée, d'environ $140 \mathrm{~mm}$ de côté. 
81 Les morphologies sont variables, avec perforation latérale ou non. La perforation permet la circulation d'air chaud entre les différentes colonnes de tubuli et une meilleure diffusion latérale de la chaleur. La forme de la perforation elle-même est variable, de forme carrée ou circulaire. Dans certains cas, elle est ébauchée mais non réalisée : de telles observations ont été faites à la "villa des Preux » à Saint-Herblain (Barraud, 1979) et à Rezé, « quartier Saint-Lupien ».

Dans la plupart des éléments étudiés ici, sur les grands côtés, c'est-à-dire ceux en contact avec la maçonnerie ou l'enduit interne, des marques d'accroche sont présentes pour favoriser l'adhérence avec le mortier (Haute-Goulaine, Piriac-sur-Mer et Rezé). Ces marques (stries réalisées au peigne) prennent des formes variées, losangiques ou ondées, avec de nombreuses combinaisons possibles.

\section{Les tegulae mammatae}

Elles restent assez rares dans la région, puisque seuls trois sites ont livré des restes, Haute-Goulaine, "Les Cléons "; Saint-Michel-Chef-Chef, "La Pouplinière » (Nauleau, 2011) et Saint-Florent-le-Vieil, "Lotissement ouest » (Nauleau dans Viau, à paraître), mais aucun élément intact ne nous est parvenu (fig. 18).

Figure 18 : Les tegulae mammatae.

Figure 18: Tegulae mammatae.

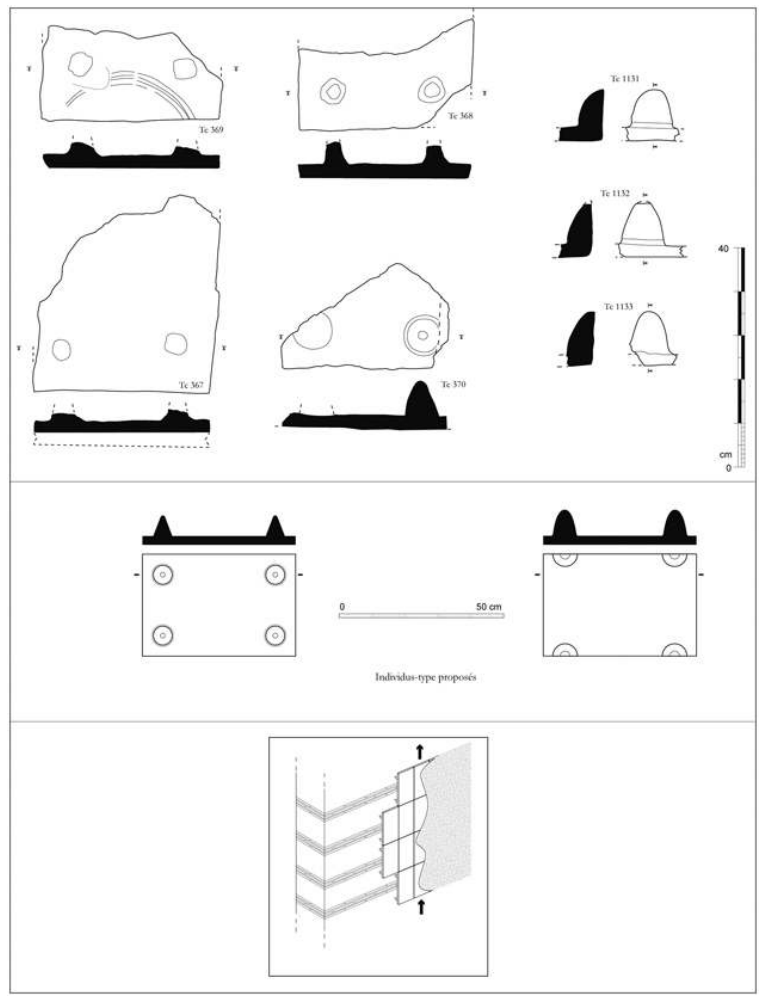

Tc 367, 368, 369, 370 : Haute-Goulaine « Les Cléons » ; Tc 1131, 1132, 1133 : Saint-Florent-le-Vieil "Lotissement Ouest ».

Tc 367, 368, 369, 370 : Haute-Goulaine "Les Cléons"; Tc 1131, 1132, 1133 : Saint-Florent-le-Vieil "Lotissement Ouest".

Il s'agit d'une brique quadrangulaire assez peu épaisse, de 22 à $28 \mathrm{~mm}$, munie de quatre plots disposés dans les angles. Aux «Cléons", un format a pu être reconstitué, 
d'environ 485 x $320 \mathrm{~mm}$, les plots coniques étant longs de 60 à $65 \mathrm{~mm}$. Deux familles de matériaux ont pu être identifiées à partir de la morphologie des plots. On rencontre un type avec plots minces façonnés grossièrement $(\mathrm{Tc} 367,368,369)$ et un type avec plots plus trapus, coniques et très bien façonnés (Tc 370, 371, 372).

Les exemplaires du site de Saint-Florent-le-Vieil sont sensiblement différents. Les plots sont semi-coniques et disposés en bordure de la brique. Ce modèle précis ne trouve pas d'équivalent dans la littérature dédiée à ces matériaux, mais des modèles assez proches existent (Hoffmann, 1975 ; De Filippo, 2004 ; Degbomont, 1984).

Les plots étant souvent désolidarisés de la brique, seule la zone d'arrachement de ceuxci permet de reconnaître ces matériaux et de les différencier d'une simple brique. C'est le cas à Saint-Michel-Chef-Chef, sur le site de "La Pouplinière", où ces matériaux n'avaient pas été identifiés dans un premier temps.

Mise en œuvre à la verticale contre une maçonnerie, cette brique permettait de constituer des parois creuses dans lesquelles circulait l'air chaud en provenance de l'hypocauste. Il n'est pas impossible que ces éléments aient pu être utilisés dans la constitution de cloisons creuses, dans le but de déshumidifier et d'assainir les murs en l'absence d'hypocauste (Adam, 2008).

\section{Les briques de placage}

88 Ces briques sont relativement rares et n'ont été rencontrées qu'en contexte de probable villa, aux «Cléons ", à Piriac-sur-Mer et à Jard-sur-Mer. Il s'agit de briques peu épaisses, portant systématiquement des incisions géométriques sur une de leurs faces. Ces incisions, qui sont parfois interprétées comme des décors, ne sont en réalité que des marques d'accroche permettant l'adhérence de la brique au mortier. Ces stries ne sont jamais rencontrées sur les briques de maçonnerie traditionnelle. Elles sont en revanche assez similaires à celles réalisées sur les tubulures. Cette constatation, ajoutée à la faible épaisseur de ces matériaux, permet de penser qu'elles étaient mises en œuvre à la verticale. Les épaisseurs varient de 16 à $21 \mathrm{~mm}$ pour les exemplaires de Piriac-sur-Mer, jusqu'à $30 \mathrm{~mm}$ pour ceux des "Cléons ». Le format de ces matériaux nous est inconnu, faute d'individus complets. Une seule largeur de $100 \mathrm{~mm}$ est conservée sur l'élément des « Cléons ». Les individus de Piriac-sur-Mer sont assurément de plus grande taille.

Les marques d'accroche sont plus ou moins prononcées et de facture assez variable. Elles sont très organisées et géométriques à Piriac-sur-Mer, et sont soit des lignes orthogonales soit des lignes obliques superposées, ou encore des motifs ondés concentriques, probablement réalisés au peigne (fig. 19, 20). Un dernier cas cumule lignes ondées puis lignes parallèles. L'exemplaire de Jard-sur-Mer est également doté de lignes ondées, mais réalisées avec un peigne à dents moins nombreuses. 
Figure 19 : Les briques de placage.

Figure 19 : Cladding bricks.

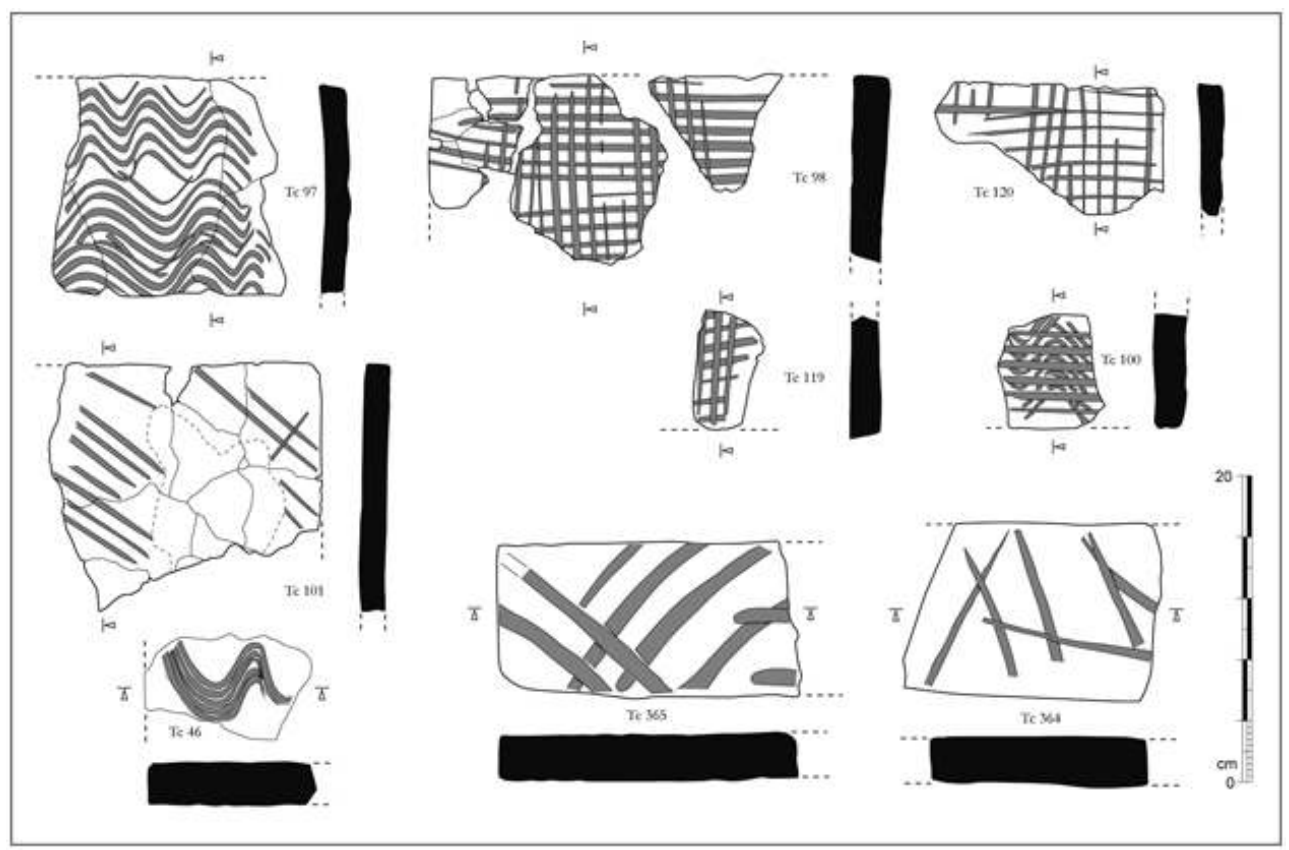

Tc 46 : Jard-sur-Mer « Le Grand Essart »; Tc 97, 98, 100, 101, 119, 120 : Piriac-sur-Mer « ZA du Pladreau »; Tc 364, 365 : Haute-Goulaine « Les Cléons ».

Tc 46: Jard-sur-Mer "Le Grand Essart"; Tc 97, 98, 100, 101, 179, 120 : Piriac-sur-Mer "ZA du Pladreau" ; TC 364, 365 : Haute-Goulaine "Les Cléons".

Figure 20 : Brique de placage, Piriac-sur-Mer « ZA du Pladreau ».

Figure 20 : Cladding brick, Piriac-sur-Mer "ZA du Pladreau".

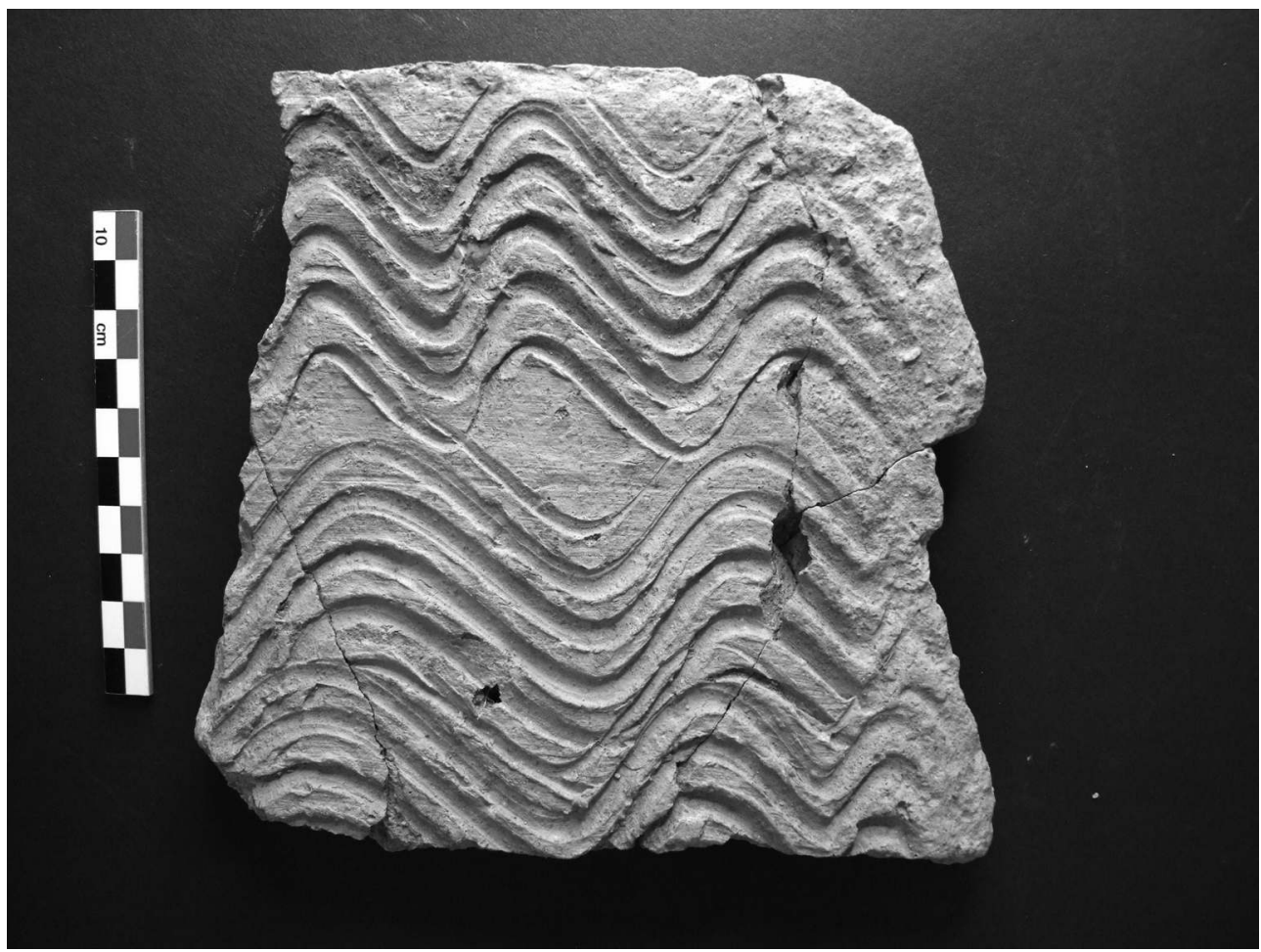

Cliché J.-F. Nauleau, Inrap. 
mise en œuvre à la verticale de ces matériaux est quasi avérée, leur place architecturale reste à préciser. Traditionnellement, on considère que ces briques sont associées à des bobines de terre cuite et des fiches métalliques en forme de $\mathrm{T}$ permettant la constitution de parois creuses, les bobines servant d'entretoise entre la maçonnerie et ces briques de placage, et ce dispositif remplissant alors le rôle des tubulures et des tegulae mammatae. Les marques d'accroche seraient alors destinées à améliorer l'adhérence des enduits en interne. Des bobines et des briques de placage de ce type ont été rencontrées dans les thermes d'Entrammes (Naveau, 1992), mais dans l'aire d'étude, aucune bobine n'a été découverte en association avec les briques rencontrées.

Sur le site de Barzan (17), de nombreuses briques de ce type ont été découvertes en contexte thermal. L'hypothèse retenue est celle de briques mises en œuvre dans un plafond plat suspendu, maintenu ici aussi par des crampons métalliques en $\mathrm{T}$, retrouvés en abondance (Bouet, 2003, p. 175).

D'autres hypothèses ont été avancées, qui suggèrent dans certains cas qu'il s'agit de briques décorées mises en œuvre au sein de bandeaux horizontaux ou de plinthes (Bouet, 1999, p. 165 : thermes de Gaujac [30]). L'hypothèse la plus probable reste celle de matériaux participant à la constitution de cloisons ou de plafonds creux, mais, en l'absence d'un éventail de données plus large, on ne peut malgré tout exclure que ces briques aient été utilisées dans d'autres contextes.

93 Il est difficile de savoir si cette technique de constitution de parois creuses a coexisté avec les autres techniques plus répandues que sont les tubuli et les tegulae mammatae ou si elles se sont succédé dans le temps. À Entrammes, ces briques de placage et bobines coexistent avec des tubuli, dans des pièces différentes. J. Naveau constate que les briques sont mises en œuvre dans la salle la moins chaude (Naveau, 1992, p. 151). On considère en général que les tegulae mammatae ont précédé les tubuli, ceux-ci apparaissant au cours du $\mathrm{I}^{\mathrm{er}}$ siècle de notre ère (Adam, 2008, p. 292). Le dispositif constitué de bobines et de briques de placage serait chronologiquement intermédiaire aux deux précédents, mais notons que des tegulae mammatae ont été trouvées en contexte des II $^{\mathrm{e}}$-III ${ }^{\mathrm{e}}$ siècles apr. J.-C. (Le Bot, 2003, p. 15).

\section{Les briques échancrées}

\section{Échancrure semi-hexagonale}

Il s'agit de briques munies d'une découpe semi-hexagonale (à trois pans) sur leurs deux grands côtés (fig. 22). Elles sont rares ; un seul élément ayant été mis au jour sur le site de production des Clouzeaux. Il s'agit probablement d'un raté de cuisson. Il mesure $325 \times 255 \mathrm{~mm}$ et est épais d'environ $35 \mathrm{~mm}$. Un autre exemplaire incomplet se trouve au musée des beaux-arts d'Angers, dont les dimensions restituées sont de 325 x $310 \mathrm{~mm}$. Il provient vraisemblablement du site de Sainte-Gemmes-sur-Loire " Les Châtelliers de Frémur » (Maine-et-Loire, fouille Godard-Faultrier de 1871 à 1875). Sur ce site, les briques ont été trouvées en place dans les thermes, formant des conduits en connexion avec un hypocauste et semblant jouer le rôle de tubulures le long des maçonneries (fig. 21). L'impossibilité de réaliser une élévation à joints croisés avec ce seul type de matériau est manifeste. Aussi, la nature de l'élévation au-dessus de celui-ci reste inconnue. D'ailleurs, la constitution de maçonneries à partir de ce seul type de brique présenterait bien peu d'avantages par rapport à l'usage des matériaux habituels

Revue archéologique de l'Ouest, 30 | 2013 
(tubulures, tegulae mammatae ou briques de placage). Elles sont profondes et occasionneraient une perte de volume importante dans la pièce et, par ailleurs, elles seraient bien peu efficaces en termes de rapidité d'acquisition et de transfert de chaleur dans les pièces qui en seraient pourvues. Notons que d'autres briques de ce type sont décrites à Poitiers dans un contexte qui semble similaire mais tout aussi peu documenté (Farago-Szekeres, Duday, 2008).

Figure 21 : Sainte-Gemmes-sur-Loire "Les Châtelliers de Frémur » / thermes. Figure 21 : Sainte-Gemmes-sur-Loire "Les Châtelliers de Frémur" / baths.

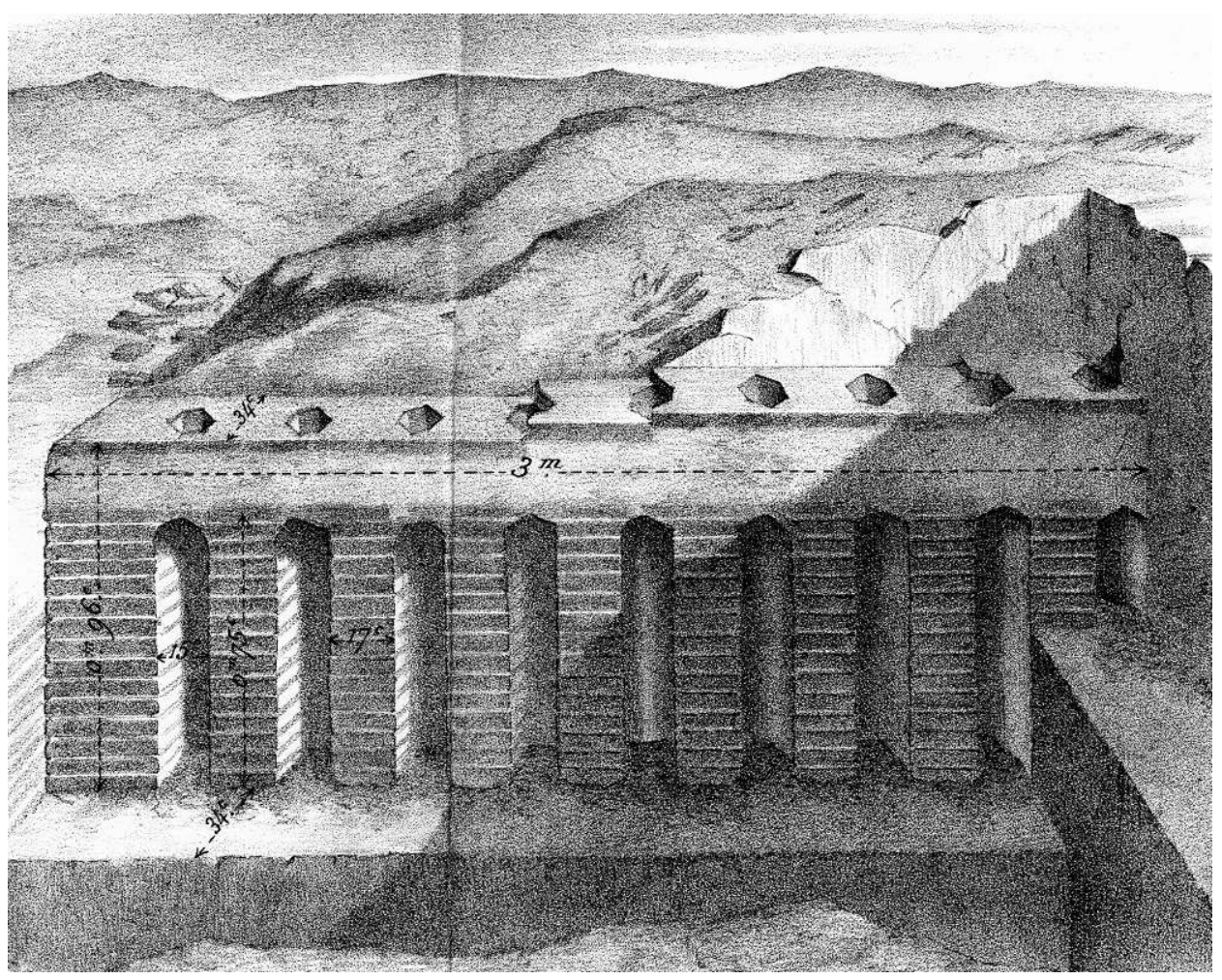

«Ep. Rom. Tubes calorifères hexagonaux trouvés en mars 1873, dans le mur nord de l'un des hypocaustes des Châtelliers de Frémur, côté occidental des bains ».

"Ep. Rom. Hexagonal heating tubes hexagonaux found in march 1873, in the north wall of one of the hypocausts at Châtelliers de Frémur, west side of the baths".

Base Joconde, ministère de la Culture. 
Figure 22 : Les briques à échancrures semi-hexagonales (Tc 628 : Les Clouzeaux « La Grosse Pierre $")$.

Figure 22 : Semi-hexagonal notched bricks (Tc 628 : Les Clouzeaux “La Grosse Pierre”).

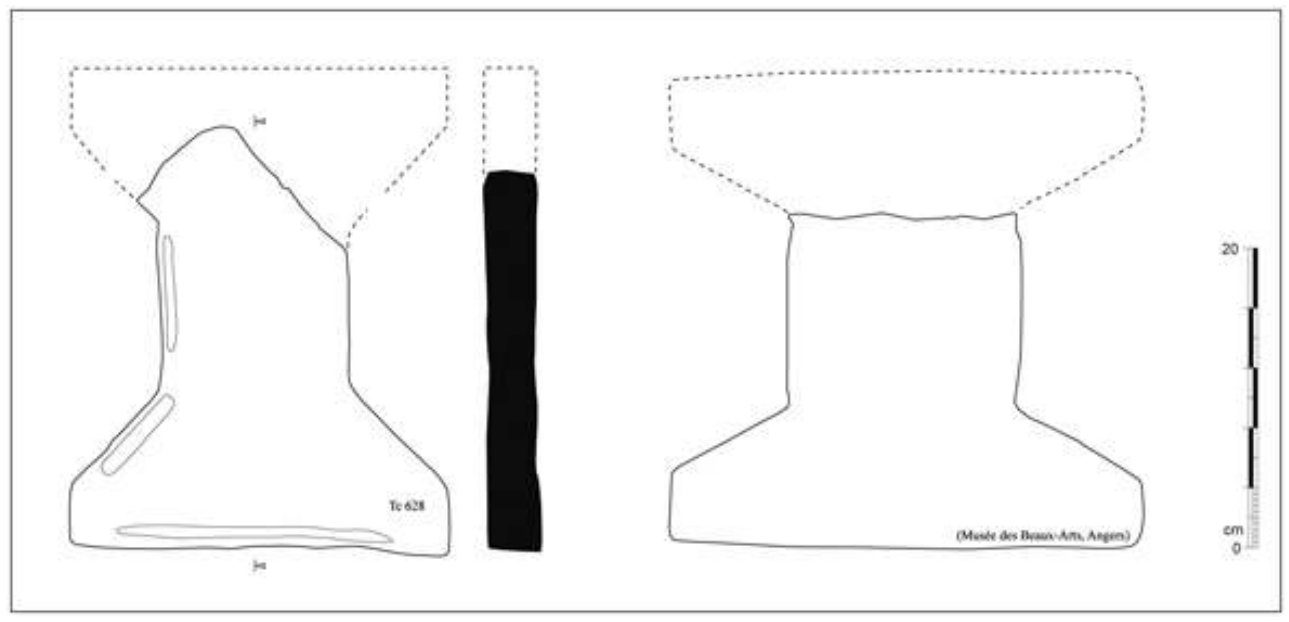

\section{Échancrure semi-circulaire}

Ce second type de briques est muni d'une découpe semi-circulaire toujours située à environ $100 \mathrm{~mm}$ de l'angle de la brique. Aucun format complet n'a été observé. Cependant, il est fort probable qu'il s'agisse de briques munies de deux échancrures latérales situées à mi-hauteur (fig. 23). Les dimensions seraient d'environ 400 x $330 \mathrm{~mm}$. Onze fragments ont été identifiés en contexte de probables villae, dont dix exemplaires sur le site des « Cléons » et un sur celui de Piriac-sur-Mer. 
Figure 23 : Les briques à échancrures semi-circulaires.

Figure 23 : Semi-circular notched bricks.

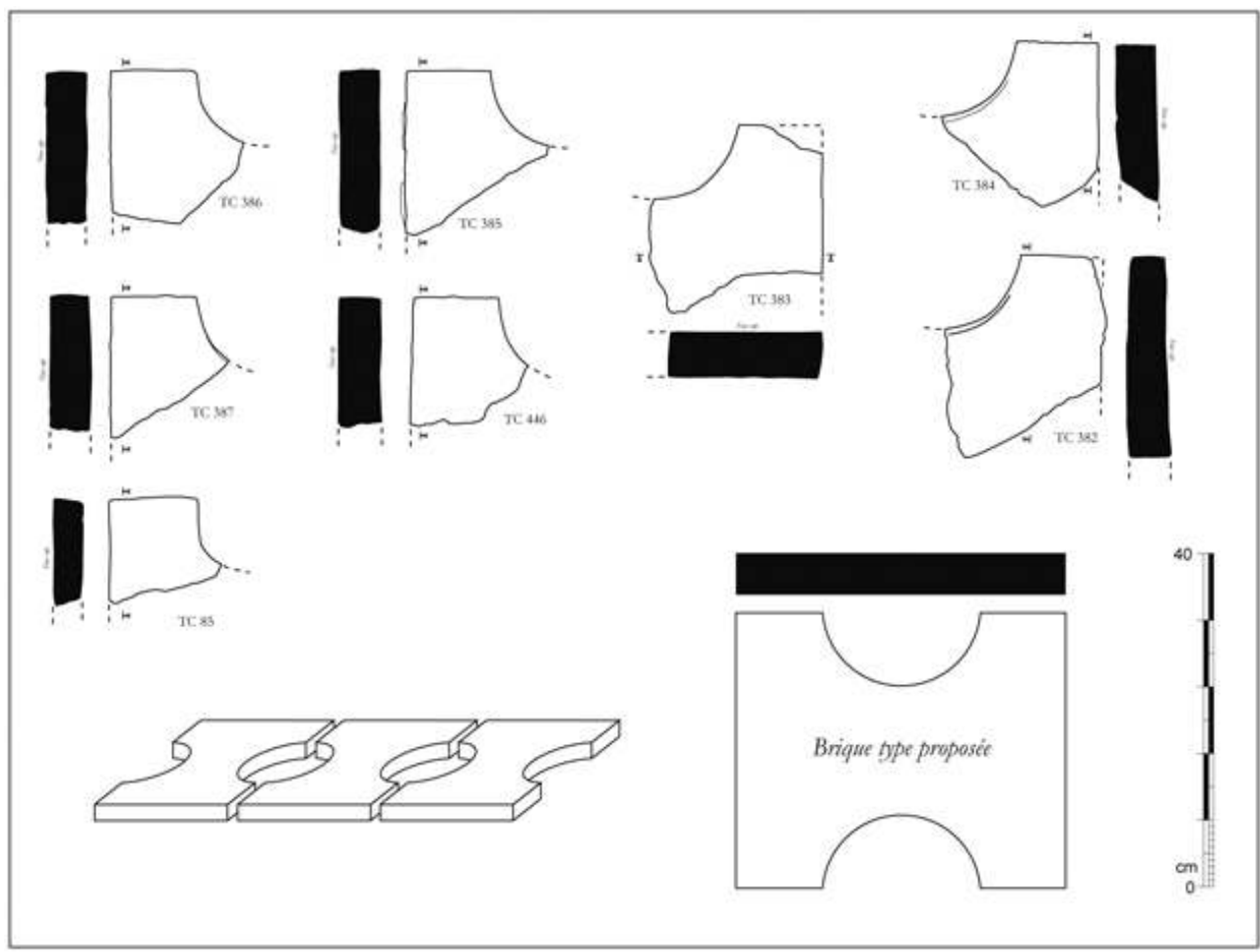

Tc 85 : Piriac-sur-Mer «ZA du Pladreau »; Tc 382, 383, 384, 385, 386, 387, 446 : Haute-Goulaine « Les Cléons ».

Tc 85 : Piriac-sur-Mer "ZA du Pladreau"; Tc 382, 383, 384, 385, 386, 387, 446 : Haute-Goulaine "Les Cléons".

Les références concernant ce type de matériau sont très rares. Notons une brique très semblable trouvée en Haute-Vienne, sur la villa de La Croisille-sur-Briance (Eygun, 1963), mais sans contexte architectural. À Rezé, dans le "quartier Saint-Lupien », le chantier-école 2012, vient de mettre au jour des briques de même famille (module des briques et taille des perforations plus réduits). Elles sont mises en œuvre dans des pilettes ${ }^{11}$ en emploi détourné ou en remploi.

Sur le site des "Cléons", F. Chaillou, qui réalisa les fouilles du site au XIXe siècle, a interprété ces éléments comme des oculli, petites fenêtres circulaires percées dans les maçonneries en contexte thermal ${ }^{12}$. Cette hypothèse peut à présent être rejetée. En effet, même si la mise en œuvre de ces éléments nous est inconnue, on peut faire un parallèle avec les éléments à échancrures semi-hexagonales décrits ci-dessus. Accolées les unes aux autres, ces briques pouvaient ménager, de la même façon que dans le premier cas, des conduits internes circulaires.

Les contextes de découverte de ces briques à découpe ne sont à l'heure actuelle pas assez documentés pour nous permettre de bien comprendre leur fonction architecturale.

\section{Les briques-claveaux}

Les briques-claveaux, mises en œuvre dans des arcs ou voûtes en dehors d'un contexte thermal, sont rares voire inexistantes. En théorie, il s'agit simplement de briques plus épaisses à une des deux extrémités (section en trapèze). Le façonnage des briques étant 
souvent de piètre qualité et les épaisseurs très variables, aucune brique-claveau n'a été clairement identifiée. Dans le monde romain, les arcs sont en général réalisés avec des briques classiques, seule la variation de l'épaisseur de mortier dans les joints à l'intrados et à l'extrados de l'arc assurant leur disposition en éventail permettant de réaliser le cintre.

100 Il existe cependant des briques spécifiques qui semblent propres à la Gaule et qui présentent un certain nombre d'encoches latérales permettant des associations avec d'autres matériaux (Fincker, 1986 ; Bouet, 1999, p. 84).

101 Ces briques, d'épaisseur constante, possèdent des encoches dans leurs angles supérieurs. Elles sont mises en œuvre dans des arcs constituant des voûtes en berceau (fig. 24). Les encoches permettent la pose de briques entre deux arcs contigus dans le but de constituer un coffrage perdu ou de ménager un espace vide qui, lorsqu'il est connecté à une file de tubulures, autorise le chauffage de cet espace et de la voûte. Ce type de brique-claveau apparaîtrait dans sa version la plus simple (en coffrage perdu) vers le milieu du $\mathrm{I}^{\text {er }}$ siècle apr. J.-C., se complexifiant à la charnière des $\mathrm{I}^{\mathrm{er}}$ et $\mathrm{II}^{\mathrm{e}}$ siècles de notre ère (De Filippo, 2004).

Figure 24 : Les briques-claveaux.

Figure 24 : Key bricks.

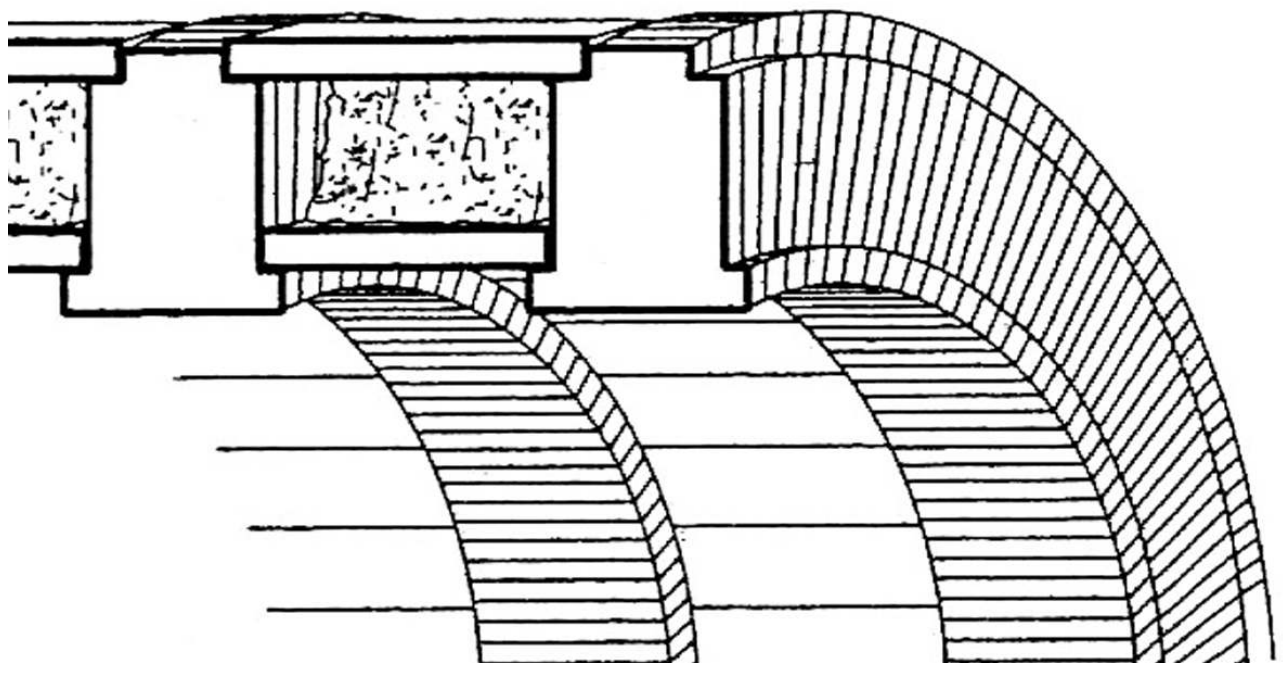



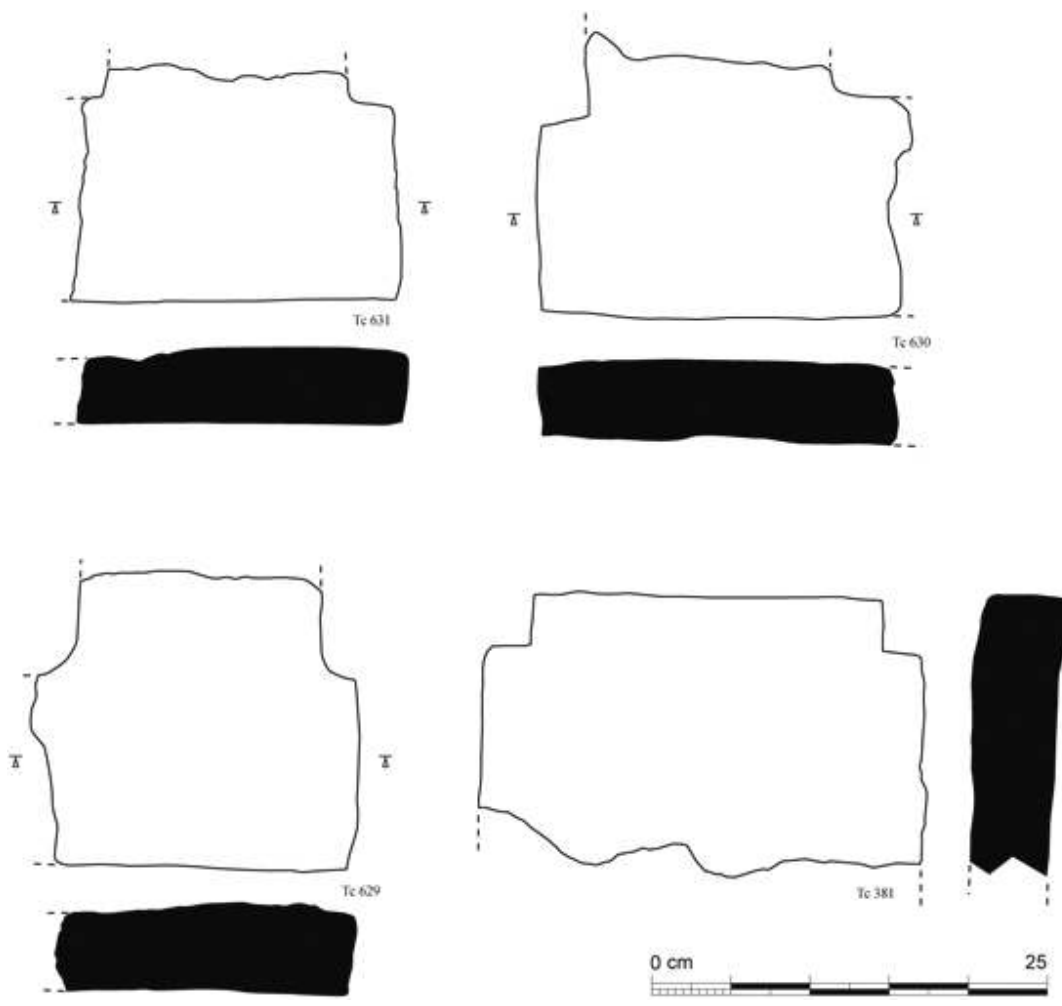

Tc 629, 630, 631 : Les Clouzeaux « La Grosse Pierre »; Tc 381 : Haute-Goulaine « Les Cléons ». Mise en contexte architectural des briques-claveaux.

Tc 629, 630, 631 : Les Clouzeaux "La Grosse Pierre"; Tc 381 : Haute-Goulaine "Les Cléons". The key bricks in their architectural context.

Dessin P. Neury, d'après C. F. Giuliani 1990, dans De Filippo 2004

Quatre exemplaires ont été dénombrés, l'un aux "Cléons » et les trois autres sur le site de production des Clouzeaux. Aux «Cléons", la brique est large de $280 \mathrm{~mm}$ et les encoches mesurent $35 \times 25 \mathrm{~mm}$.

Sur le site des Clouzeaux, on ignore s'ils étaient mis en œuvre comme tels dans la sole du four ou remployés dans ses maçonneries en tant que "ratés de cuisson ». Leur morphologie semble différente, mais la sur-cuisson dont ils ont fait l'objet complique les observations. On ne peut exclure qu'il s'agisse d'éléments spécifiques permettant de supporter la sole du four. Une étude plus poussée de ces éléments sera réalisée dans le cadre de la publication de ce site.

\section{Des tegulae sans encoches}

Ces éléments, par leur morphologie générale, pourraient s'apparenter à des tegulae, mais ils sont dépourvus d'encoches d'emboîtement (fig. 27). Leur mise en œuvre sur des toitures est donc exclue. Deux groupes se distinguent au sein de ce type de matériau: un groupe à rebord bas de type tegula (environ $50 \mathrm{~mm}$ ) et un second type au rebord nettement plus haut (jusqu'à $100 \mathrm{~mm}$ ).

\section{À rebords bas de type tegula}

Plusieurs éléments de ce type ont été mis en évidence. L'un, complet, a été découvert en place et en position de plaque d'alandier d'une structure qui semble être un séchoir (denrées alimentaires, probablement) sur le site de la villa de Jard-sur-Mer (Tc 21). Il 
présente un format quasi carré de 334 x $332 \mathrm{~mm}$, dont les rebords ne sont hauts que de $55 \mathrm{~mm}$.

De nombreux fragments ont été identifiés à Angers « Marengo » en rejets secondaires. Une longueur complète a été conservée ; elle est de $355 \mathrm{~mm}$, ce qui semble plus court que pour le module estimé des tegulae associées (entre 400 et $420 \mathrm{~mm}$ ). Les rebords sont hauts de 48 à $53 \mathrm{~mm}$, valeurs conformes à ce qui est mesuré sur les tegulae.

\section{À rebords hauts}

Deux exemplaires de tegulae sans encoche et à rebords hauts ont été identifiés. Alors que la hauteur moyenne des rebords de tegulae est comprise entre 38 et $55 \mathrm{~mm}$, ces deux exemplaires présentent des hauteurs de 72 et $100 \mathrm{~mm}$. Les deux ont été découverts en contexte de villa, le premier à Piriac-sur-Mer (Tc 76) et le second aux "Cléons » (Tc 350). À Piriac-sur-Mer, l'exemplaire est muni d'une découpe latérale que ne possède pas l'autre individu.

108 La découverte la plus remarquable a été faite récemment à Rezé dans le "quartier Saint-Lupien » (2011). Huit tegulae de ce type ont été découvertes en place, formant une canalisation destinée à évacuer les eaux pluviales au sein d'un ambitus très étroit (fig. 25, 26). Ces tegulae très homogènes mesurent de 350/359 x 325/330 mm et le rebord est haut de 82 à $90 \mathrm{~mm}$, soit le double de la hauteur classique pour une tegula. Elles ne peuvent pas s'emboîter et sont simplement posées en connexion, sans dispositif d'étanchéité particulier. Le contexte chronologique les situe dans l'intervalle 60-100 apr. J.-C.

Figure 25 : La canalisation du « quartier Saint-Lupien » à Rezé. Figure 25 : The canalisation of the "quartier Saint-Lupien" at Rezé.

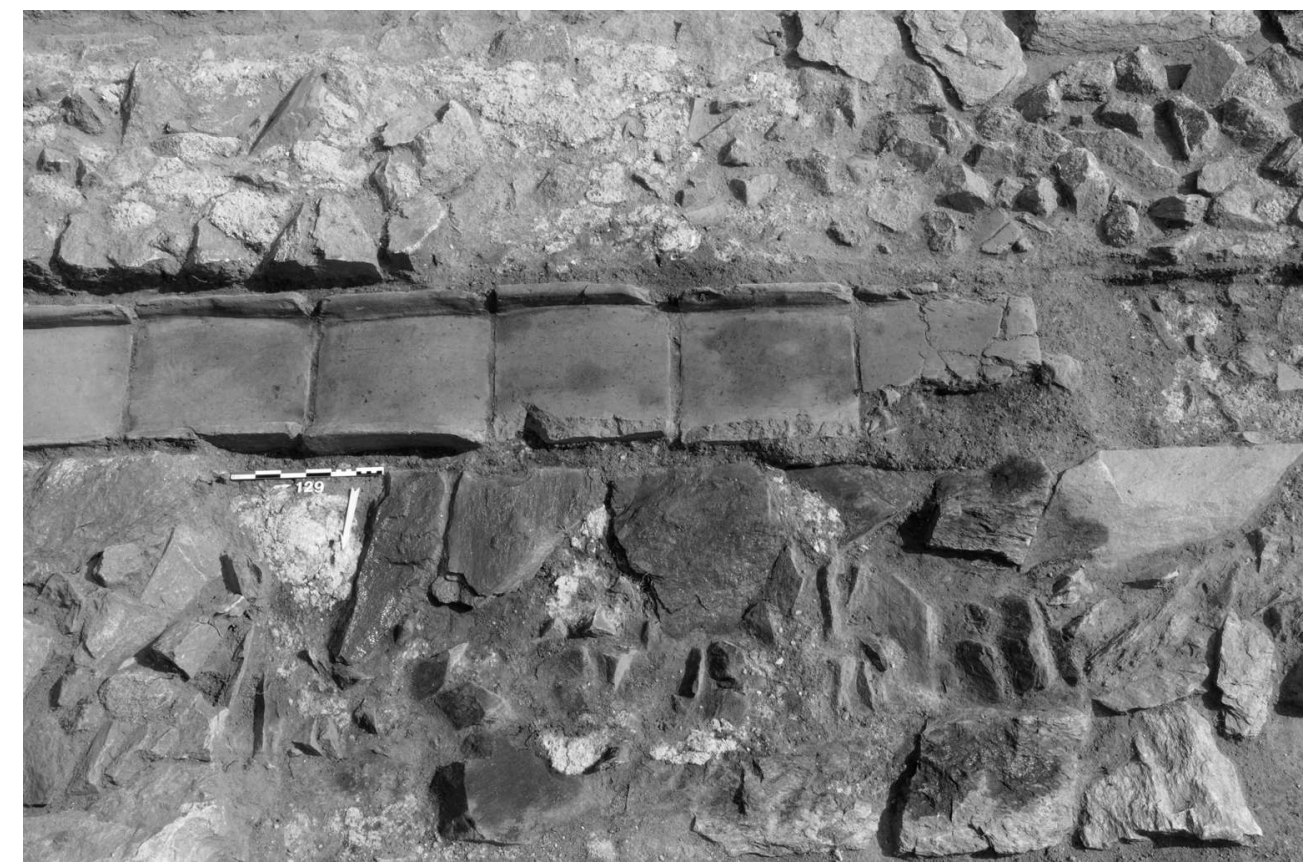

Cliché Rezé - équipe de fouille programmée 2011. 
Figure 26 : Tegula sans encoche Tc 980, « quartier Saint-Lupien » à Rezé, chantier-école 2011. Figure 26 : tegula without notch Tc 980, "quartier Saint-Lupien" à Rezé, chantier-école 2011.

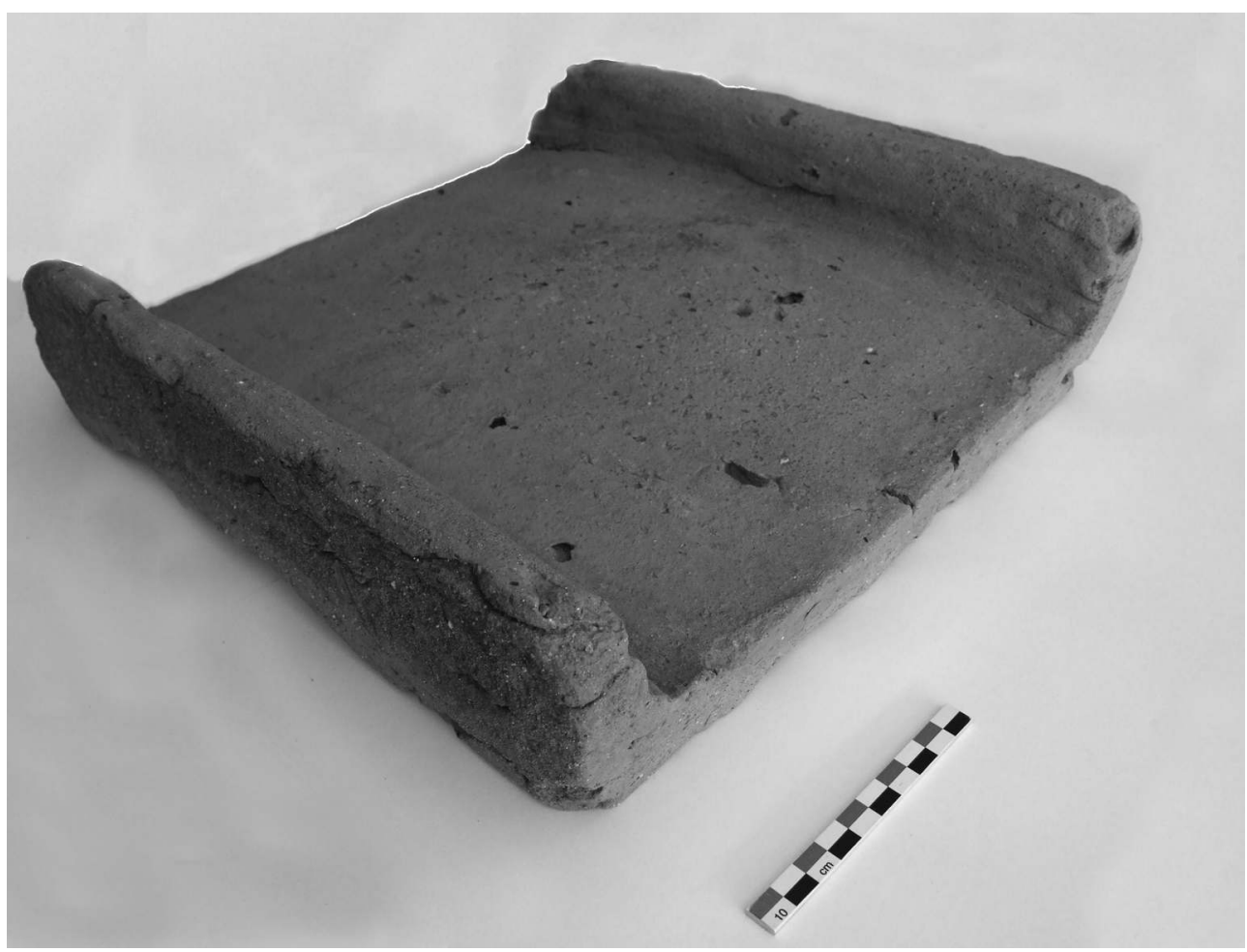

Cliché J.-F. Nauleau, Inrap.

Figure 27 : Les tegulae sans encoche.

Figure 27 : The tegulae without notches.

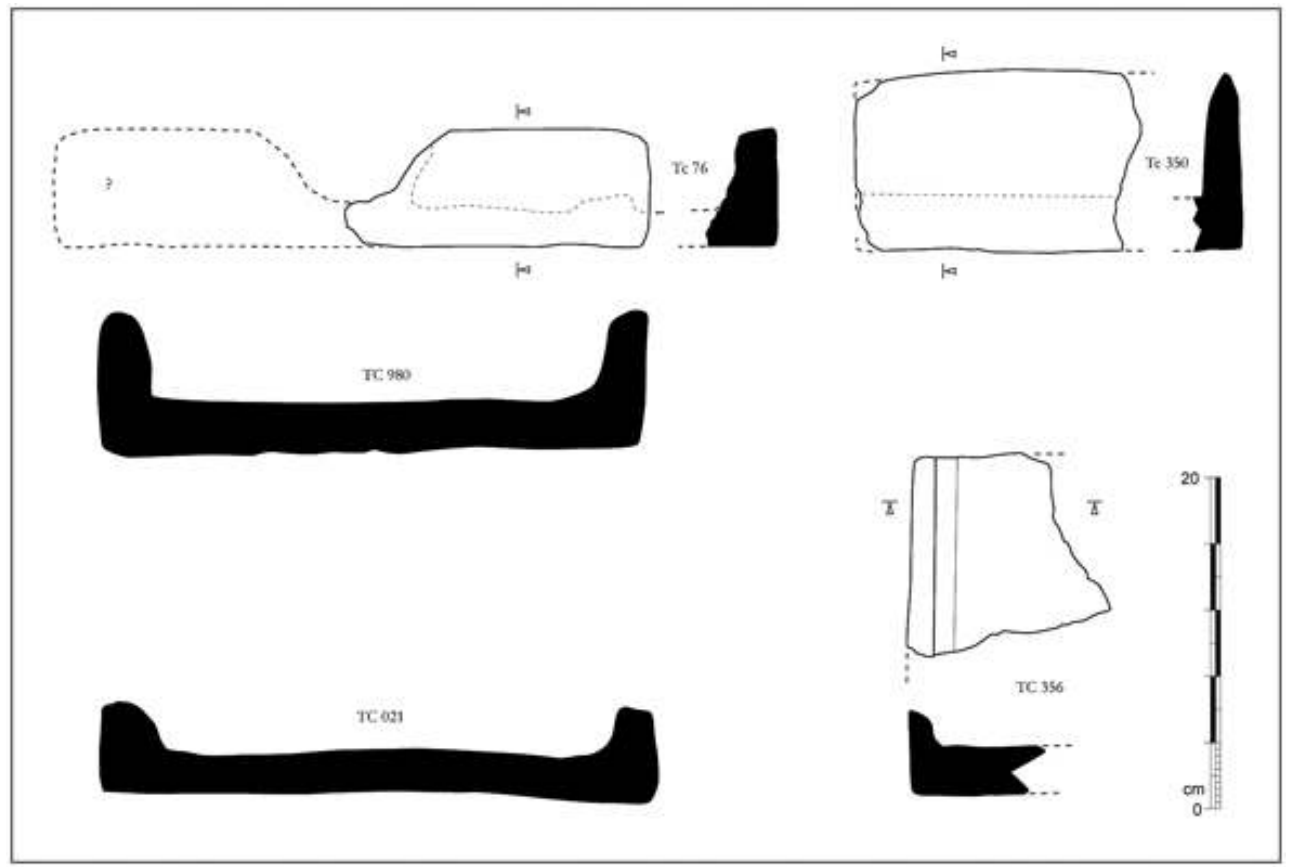

Tc 76 : Piriac-sur-Mer « ZA du Pladreau »; Tc 350, 356 : Haute-Goulaine « Les Cléons »; Tc 21 : Jardsur-Mer « Le Grand Essart »; Tc 980 : Rezé « quartier Saint-Lupien ».

Tc 76: Piriac-sur-Mer "ZA du Pladreau"; Tc 350, 356: Haute-Goulaine "Les Cléons"; Tc 27 : Jard-sur-Mer "Le Grand Essart"; Tc 980 : Rezé "quartier Saint-Lupien". 
109 À Rezé, les canalisations sont généralement réalisées en tegulae, l'usage avéré de matériaux spécifiques étant ici le seul exemple connu dans cette agglomération et plus globalement dans la région.

110 Mentionnons tout de même que des tegulae sans encoche, de 460 x $350 \mathrm{~mm}$, ont été signalées dans le Bas-Dauphiné : ces « dalles » sont placées à l'envers, posées sur une couche de sable sur un sol d'hypocauste. La hauteur des rebords est ici de $56 \mathrm{~mm}$ (Chauffin, 1956).

111 En définitive, ces tegulae sans encoche semblent avoir eu des usages multiples, puisqu'on en dénombre au moins trois de façon avérée, plaque d'alandier dans un séchoir à céréales (?), élément de canalisation ou de caniveau et élément de suspensura. Ils sont probablement encore plus variés, mais il est probable que ces matériaux spécifiques ne soient pas identifiés sur le terrain ni distingués de tegulae ordinaires, quand ils sont fragmentés.

\section{Matériaux indéterminés}

\section{Les tegulae à rebords discontinus}

112 Il s'agit de fragments d'éléments présentant une morphologie de tegula mais avec un rebord interrompu ou discontinu (fig. 28). Les données sont très semblables pour les différents individus, avec une hauteur de rebord de $80 \mathrm{~mm}$, nettement supérieure à ce qui est rencontré pour les tegulae, et des longueurs de rebord d'environ $70 \mathrm{~mm}$. La plaque est épaisse de 25 à $30 \mathrm{~mm}$, soit des valeurs légèrement supérieures à ce qui est rencontré pour des tegulae. Plusieurs éléments de ce type ont été découverts aux "Cléons». La fragmentation de ces éléments nuit à la compréhension de leur morphologie: dans certains cas, le segment de rebord est placé dans l'angle de l'élément (Tc 348, 349) ; dans d'autres, un espace est ménagé avant l'angle (Tc 345, 346, 347). 
Figure 28 : Les tegulae à rebords discontinus, variantes des tegulae mammatae? (Haute-Goulaine "Les Cléons").

Figure 28 : The tegulae discontinued edges, variants of tegulae mammatae? (Haute-Goulaine "Les Cléons").

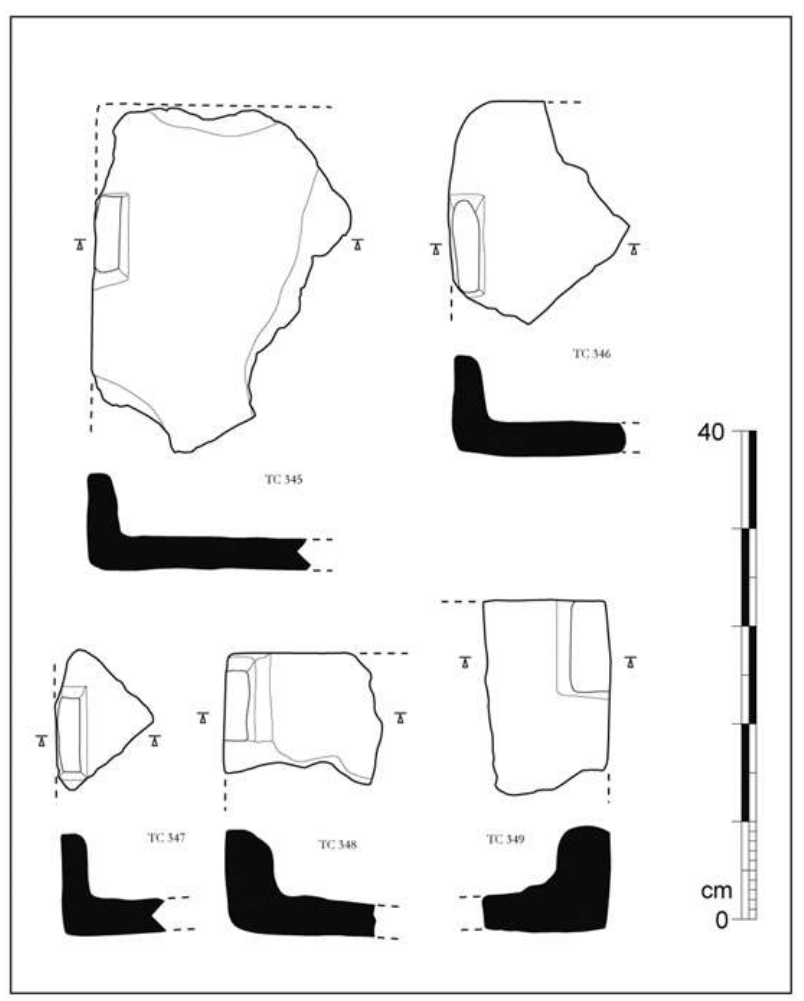

113 On peut poser comme hypothèse qu'il s'agisse d'une variante de tegulae mammatae avec plots quadrangulaires disposés dans les angles de la brique. Ce type de tegulae mammatae est avéré dans le monde romain (Adam, 2008, p. 292), mais non en Gaule. La hauteur des plots $(80 \mathrm{~mm})$ correspondrait à ce qui a été décrit précédemment pour les tegulae mammatae. Mais en l'absence de données complémentaires sur d'autres lots, la question reste pleinement ouverte.

\section{Une tegula décorée?}

Deux fragments en terre cuite atypiques et similaires (fig. 29) ont été mis au jour dans deux structures distantes de vingt-cinq mètres sur le site de Carquefou «La Haute Forêt » (Nauleau, 2012). Les deux éléments ont été découverts dans un contexte du haut Moyen Âge, mais il a été démontré que les terres cuites étaient en remploi pour cette phase chronologique sur le site ${ }^{13}$. 
Figure 29 : Les matériaux décorés indéterminés. Figure 29 : Undetermined decorated elements.

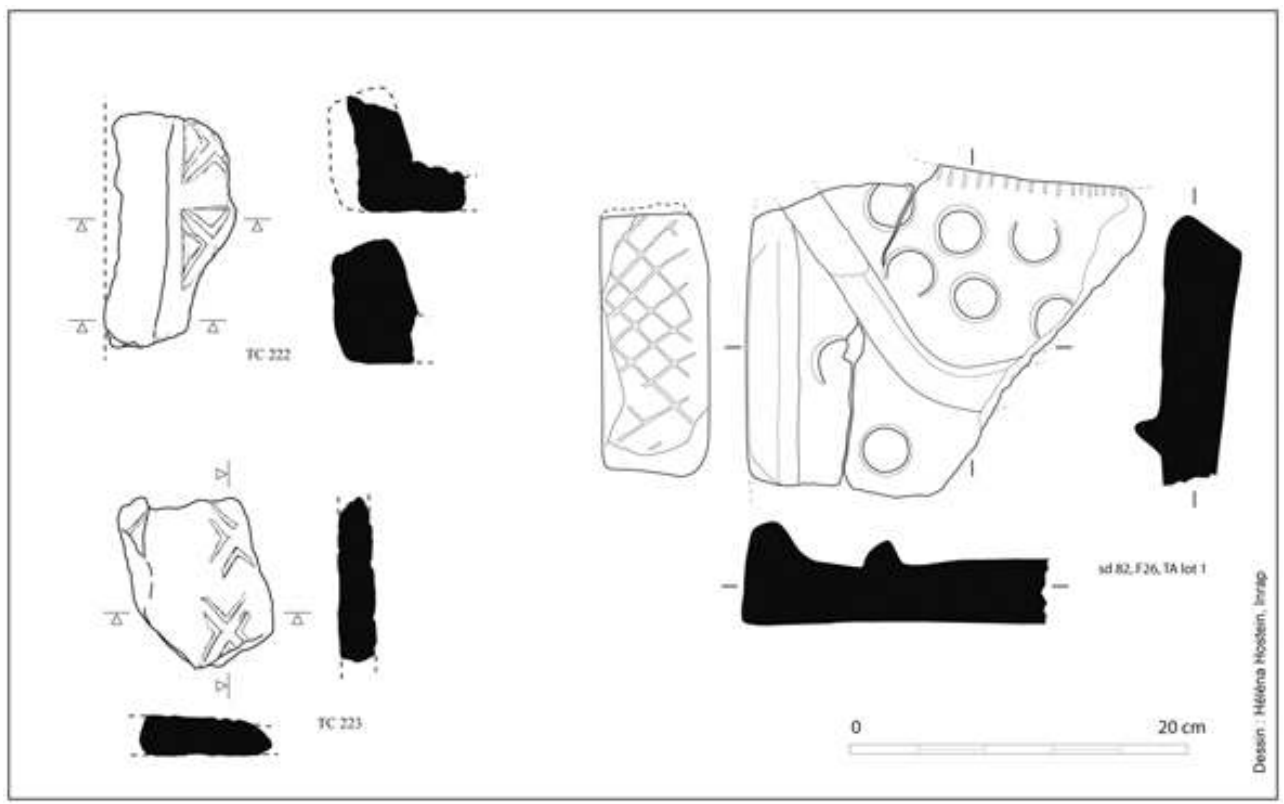

Tc 222, 223 : Carquefou " La Haute Forêt II » ; à droite : Saint-Herblain, « Zac Ar Mor ». Tc 222, 223 : Carquefou "La Haute Forêt II"; à droite : Saint-Herblain, "Zac Ar Mor".

115 Le premier individu est un fragment de ce qui semble être un rebord de tegula dont l'épaisseur de fond est de $27 \mathrm{~mm}$ et dont le rebord est haut de $70 \mathrm{~mm}$ et large à la base de $49 \mathrm{~mm}$. La tuile porte, en face supérieure, deux marques cruciformes incomplètes alignées à la base du rebord.

116 Un second fragment, qui pourrait appartenir au même individu, est un fragment de fond épais de $23 \mathrm{~mm}$ qui porte lui aussi deux traces cruciformes incomplètes et assez altérées. Les marques, qui paraissent réalisées au poinçon (par estampage), sont de même type : $46 \mathrm{~mm}$ de long pour une largeur estimée à $35 \mathrm{~mm}$.

Les dimensions du rebord de la tegula sont peu communes. Sa hauteur externe, traditionnellement comprise entre 45 et $50 \mathrm{~mm}$ est ici de $70 \mathrm{~mm}$; la largeur, ordinairement comprise entre 25 et $35 \mathrm{~mm}$, est ici de $49 \mathrm{~mm}$. En dehors des marques digitées et incisions sur tranches, qui correspondent à des marquages, des tegulae décorées n'ont jamais été signalées dans la région. Il est d'ailleurs peu probable qu'il s'agisse d'une tegula classique, ces fragments évoquant plutôt des éléments décoratifs d'un autre type qui reste à définir, le contexte de découverte (remploi) ne permettant pas de travailler davantage sur cette question ${ }^{14}$.

118 Mentionnons à ce sujet un élément découvert au cours de la fouille du site de SaintHerblain «ZAC Armor » (Mercier, 2007; fig. 29). Il s'agit d'une plaque épaisse de 35 à $45 \mathrm{~mm}$, fragmentée, munie d'un large rebord au tracé rectiligne qui semble amorcer une courbure. La partie supérieure de l'objet est décorée d'un cordon appliqué, large d'une vingtaine de millimètres et haut d'une quinzaine, qui dessine un tracé en $\mathrm{V}$ (?). La surface supérieure est rehaussée d'une dizaine de lunules ou de petits cercles complets de 29 à $33 \mathrm{~mm}$ de diamètre réalisés par estampage. La surface externe du rebord est striée d'un motif losangique évoquant des marques d'accroche. La face inférieure de l'objet porte, comme pour les briques et les tegulae, les restes du lit sableux déposé au fond du moule pour éviter l'adhérence de l'argile. 
119 Deux autres petits fragments lui sont associés, mais ils ne permettent pas de comprendre la morphologie de l'ensemble. Il est difficile d'interpréter ces objets très fragmentés : il peut s'agir de matériaux d'ornementation, qu'ils soient mis en œuvre sur toiture ou en décor de placage. Cependant, il peut s'agir également de mobilier spécifique et lié aussi au type d'activité pratiquée. Rappelons qu'ils ont été découverts sur un site ayant révélé l'existence d'un « lieu à caractère religieux ». À défaut de tholos et de statue, Jacques Santrot y voit un « dispositif cultuel» mal défini (Santrot dans Mercier, 2007, p. 58).

\section{Une évolution morphologique des tegulae?}

Des études récentes semblent montrer qu'il existe une telle évolution. La réduction du format des tegulae a ainsi été constatée dans le sud de la Gaule (Feugère, 2000), tandis qu'une évolution de la forme des encoches dans le centre-est de la Gaule a également été mise en évidence (Clément, 2009), permettant, d'après l'auteur, de constituer une chrono-typologie de référence. En Grande-Bretagne, Peter Warry, dans une vaste étude, a pu corréler ces données et montrer que le gabarit ainsi que l'ampleur des encoches d'emboîtement décroissaient toutes avec le temps et dans une même mesure (Warry, 2006). Pour ce qui concerne notre aire de recherche, aucune étude globale n'a, à ce jour, été réalisée : on ne possède donc pas de cadre de référence.

La base constituée ces dernières années permet de disposer de données concernant près de trois cents tegulae dont 171 exemplaires complets ou archéologiquement complets. Cependant, on ne possède pas de données chronologiques pour l'ensemble des individus. Le contexte archéologique de découverte est essentiel : on doit en effet pouvoir identifier clairement les matériaux qui ont fait l'objet de remploi, afin de ne pas brouiller les données chronologiques.

On se référera ici aux deux sites sur lesquels les contextes chronologiques sont suffisamment précis pour traiter de cette question : la fouille d'Angers "Saint-Louis » (Nauleau dans Brodeur, à paraître) et les fouilles du «quartier Saint-Lupien » à Rezé, des années 1980 à nos jours.

\section{Les dimensions des tegulae}

La longueur des tegulae enregistrées dans la base s'échelonne entre 335 et $525 \mathrm{~mm}$.

À Angers, l'étude porte sur 85 individus dont les longueurs varient de 350 à $525 \mathrm{~mm}$ (fig. 30). Ils ne se répartissent pas de manière équilibrée sur la courbe (surabondance des contextes de la fin du $\mathrm{II}^{\mathrm{e}}$ au milieu du III $^{\mathrm{e}}$ siècle apr. J.-C.) mais ils permettent d'esquisser des tendances. On constate une assez nette évolution qui tend à la réduction la taille des tegulae dans le temps. On passe de tuiles proches ou supérieures à $500 \mathrm{~mm}$ au début du $\mathrm{I}^{\mathrm{er}}$ siècle apr. J.-C. à des individus d'environ $360 \mathrm{~mm}$, voire moins, à la fin du $\mathrm{II}^{\mathrm{e}}$ - milieu du III $^{\mathrm{e}}$ siècle apr. J.-C. Les quelques contextes intermédiaires (notamment US 3703) marquent clairement cette décroissance. Au-delà du début du III ${ }^{\mathrm{e}}$ siècle apr. J.C., les données sont plus floues, dans la mesure où il est possible que la plupart des individus étudiés dans la zone du mithraeum provient de pratiques de remplois. Cependant, la présence de tuiles de gabarit similaire à celui des tuiles du $\mathrm{III}^{\mathrm{e}}$ siècle 
apr. J.-C., mais avec des encoches basses très différentes (Tc 915), pourrait prouver l'existence d'une production au IV siècle apr. J.-C.

Figure 30 : Exemples de l'évolution de la longueur des tegulae pendant les premiers siècles de notre ère sur les sites du " quartier Saint-Lupien » à Rezé et de la fouille de « Saint-Louis » à Angers. Figure 30 : examples of the evolution of the length of tegulae during the first centuries $A D$ on the sites of "quartier Saint-Lupien" at Rezé and the excavation of "Saint-Louis" at Angers. 
Figure 31 : Des tegulae précoces à encoche basse atypique (Angers "Saint-Louis »). Figure 31 : Early tegulae with atypical low notches (Angers "Saint-Louis").

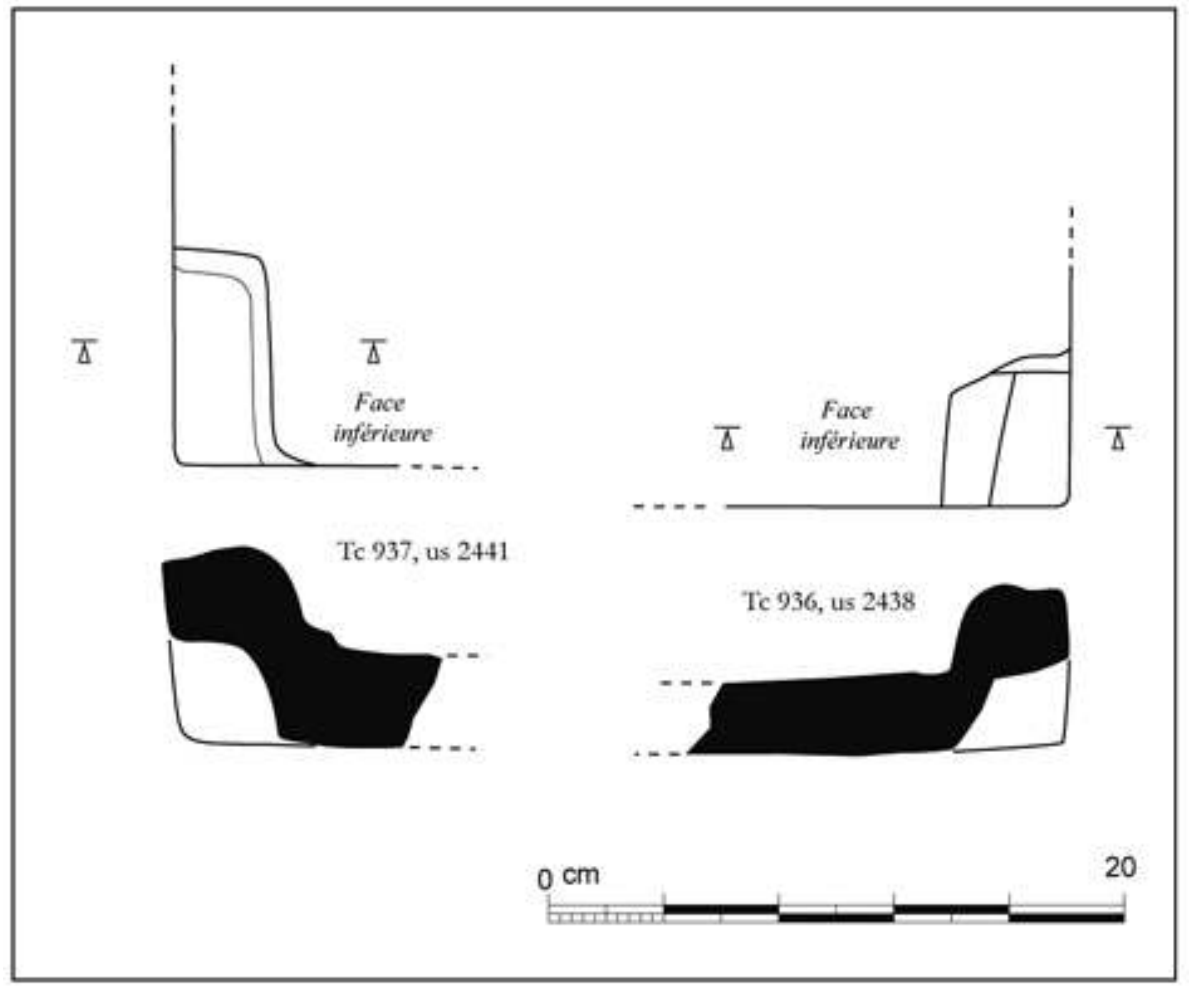

Figure 32 : Étude de la corrélation entre la taille des tegulae et les caractères morphologiques principaux à partir des tegulae complètes de la base de données TCA.

Figure 32 : Correlation between the dimensions of the tegulae and their main morphological characteristics using only complete tegulae in the TCA database.

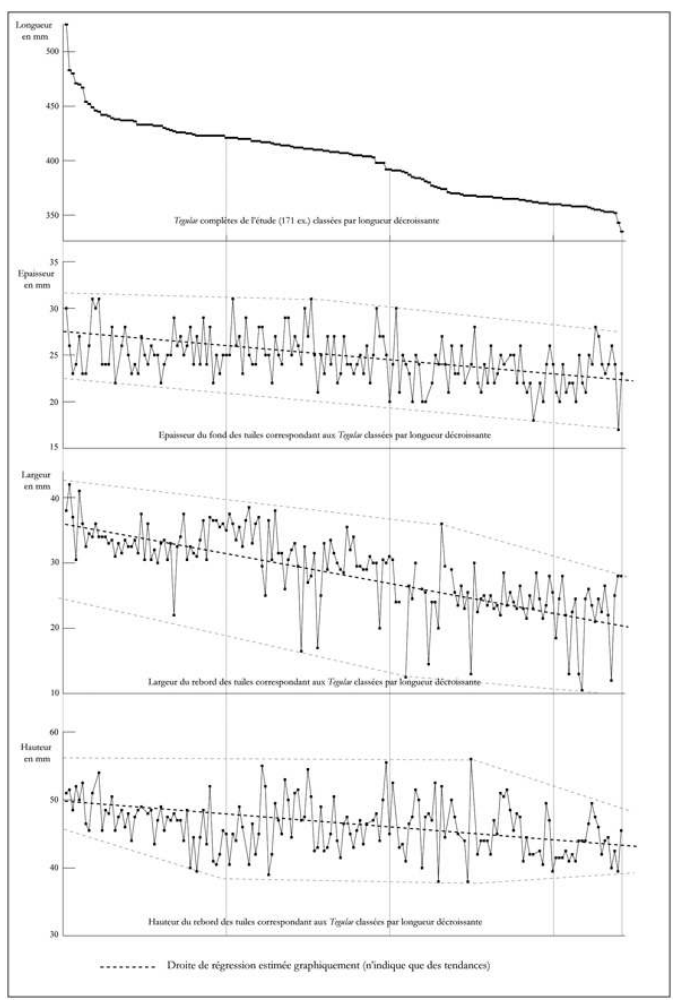


Figure 33 : Étude de la corrélation entre la taille des tegulae et la morphologie des encoches d'emboîtement des tegulae complètes de la base de données TCA.

Figure 33 : Correlation between the dimensions of the tegulae and the morphology of the interlocking notches using only complete tegulae in the TCA database.

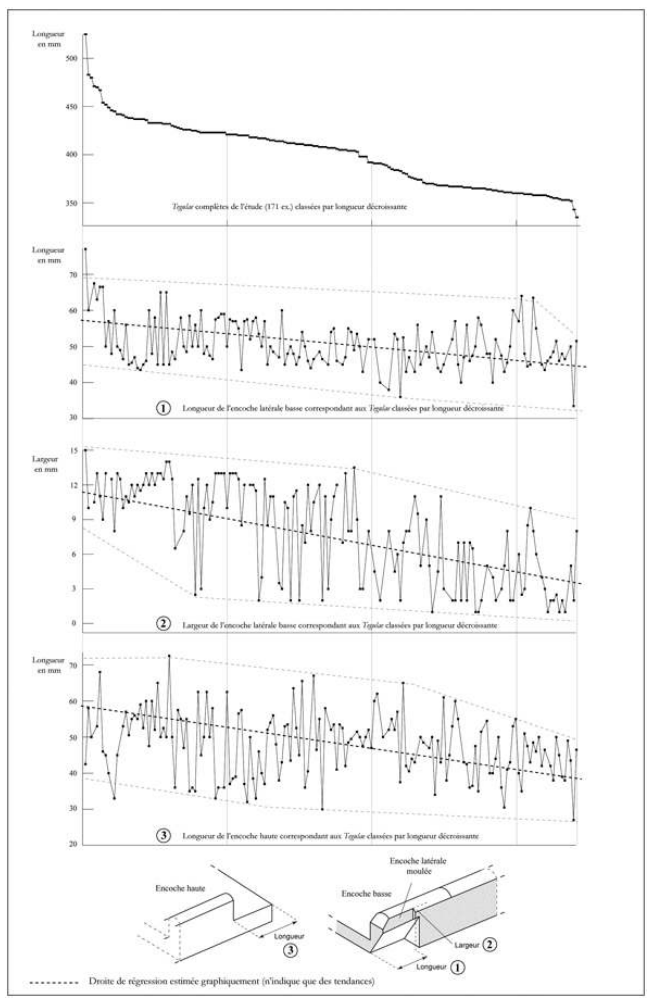


Figure 34 : Étude de la corrélation entre la taille des tegulae et le rapport longueur/largeur des tegulae complètes de la base de données TCA.

Figure 34 : Correlation between the dimensions of the tegulae and the length/width relation using only complete tegulae in the TCA database.

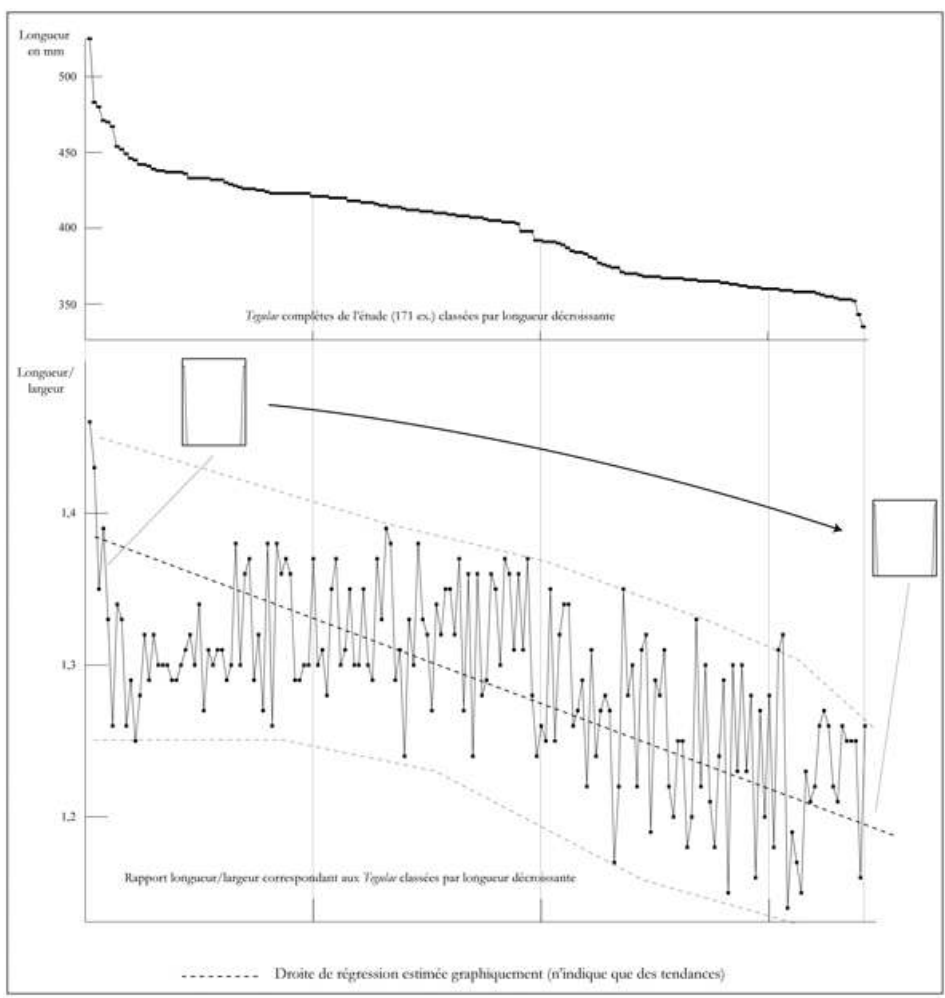

Ces courbes montrent à l'évidence que tous ces caractères sont incontestablement corrélés. La diminution de la longueur des tegulae s'accompagne de la diminution du volume de la réserve latérale de l'encoche aval, ainsi que de la diminution de l'épaisseur des tuiles et de la taille des rebords.

\section{L'étude des encoches}

\section{Les encoches aval}

Leur morphologie est toujours identique, avec une réserve latérale moulée et une encoche oblique découpée en face inférieure, qui vient oblitérer plus ou moins complètement la réserve moulée (fig. 3). De très rares exceptions à ce type d'encoche, observé à plusieurs milliers de reprises, sont à signaler. Sur le site d'Angers "SaintLouis ", deux encoches singulières ont été signalées supra dans des niveaux datant des toutes premières décennies de notre ère. Il s'agit dans les deux cas d'encoches importantes, réalisées en face inférieure et sans réserve latérale. L'une d'elles est moulée et l'autre découpée (fig. 31).

es données issues de la base concernant les encoches aval apportent des informations permettant d'orienter l'étude. Concernant la réserve moulée, les variations constatées entre les deux côtés sont très faibles, contrairement à la découpe oblique qui semble soumise à la singularité du geste du tuilier. De ce fait, l'étude portera sur la morphologie de cette encoche moulée.

L'examen de cette réserve latérale montre une nette diminution de sa longueur et de sa largeur. Pour les tuiles les plus grandes, les longueurs sont comprises entre 50 et 
$70 \mathrm{~mm}$, avec une valeur moyenne légèrement inférieure à $60 \mathrm{~mm}$. Pour les tuiles plus petites, ces valeurs passent respectivement à 35,55 et $45 \mathrm{~mm}$. En ce qui concerne la largeur de cette réserve, la tendance est la même et l'on évolue de valeurs comprises entre 9 et $15 \mathrm{~mm}$ à des valeurs entre 1 et $8 \mathrm{~mm}$, la moyenne passant environ de 11 à $4 \mathrm{~mm}$.

\section{L'encoche amont}

Elle suit une même évolution que l'encoche aval, est comprise entre 45 et $70 \mathrm{~mm}$ pour les tuiles les plus grandes et passe ensuite à des valeurs de 30 à $50 \mathrm{~mm}$ pour les plus petites. La moyenne chute de 55 à $40 \mathrm{~mm}$. On constate donc clairement que la taille des encoches diminue en même temps que le gabarit des tegulae.

\section{L'épaisseur de la tuile}

Les variations d'épaisseur sur une même tuile sont considérables, d'une part entre partie aval et amont, d'autre part entre le centre de la tuile et les parties proches du rebord. Ces variations sont fréquemment de 2 à $5 \mathrm{~mm}$. L'épaisseur des tegulae demeure donc un critère difficile à exploiter. Malgré ces difficultés, cette épaisseur semble décroître assez nettement en parallèle des autres caractères déjà évoqués. Ici, la valeur retenue est l'épaisseur mesurée en partie basse (la plus épaisse). Elle évolue entre une fourchette de $23 / 32 \mathrm{~mm}$ et $18 / 27 \mathrm{~mm}$, la moyenne passant de 27 à $22 \mathrm{~mm}$.

\section{L'étude des rebords}

$138 \mathrm{Au}$ sein du lot de tegulae étudié, la largeur des rebords est systématiquement décroissante de bas en haut afin de faciliter l'emboîtement de la tuile amont sur la tuile aval. Par ailleurs, on constate souvent des variations importantes dans la morphologie des rebords entre les deux côtés de la tuile. C'est le cas de sa largeur qui peut présenter des valeurs sensiblement différentes (la moyenne constatée est de 2,8 $\mathrm{mm}$, mais peut être supérieure à $10 \mathrm{~mm}$ ). Néanmoins, malgré ces réserves, la largeur du rebord est corrélée aux autres caractéristiques et décroît nettement.

Ici, la largeur étudiée est celle aval (la plus importante) : elle oscille entre 28 et $43 \mathrm{~mm}$ pour les plus grandes tuiles et entre 15 et 27 pour les plus petites. La valeur moyenne chute de 37 à $22 \mathrm{~mm}$. La hauteur du rebord est le seul des caractères morphologiques qui varie peu. Elle passe en moyenne de 50 à $44 \mathrm{~mm}$, mais avec une grande dispersion des valeurs.

\section{Le rapport longueur/largeur}

Ce rapport est très variable au sein du lot de tuiles étudié puisqu'il varie de 1,15 à 1,40. Ce rapport est, lui aussi, clairement corrélé à la taille des tuiles (fig. 34) : les valeurs les plus grandes sont rencontrées pour les tuiles les plus grandes, et les valeurs les plus faibles sont associées aux tuiles les plus courtes. Les tuiles longilignes du début du $\mathrm{I}^{\mathrm{er}}$ siècle apr. J.-C. tendent donc à devenir de plus en plus trapues au cours des siècles suivants. Cette tendance semble se confirmer pour le haut Moyen Âge (cf. § « Synthèse des résultats »). 


\section{Le poids des toitures} carré d'une toiture (cf. § «Les tegulae »). La diminution de l'épaisseur des tuiles induit une réduction importante du poids des toitures. On passe environ de $95 \mathrm{~kg} / \mathrm{m}^{2}$ au début $\mathrm{du} \mathrm{I}^{\mathrm{er}}$ siècle apr. J.-C. à $70 / 75 \mathrm{~kg} / \mathrm{m}^{2}$ vers le milieu du II siècle, ce qui constitue un net progrès technique en l'espace d'un siècle et demi ${ }^{16}$ (tabl. 2).

Tableau 2 : Poids de différentes toitures restituées.

Table 2 : Weight of the rendered roofs.

\begin{tabular}{|l|c|c|c|}
\hline \multicolumn{1}{|c|}{ Provenance des tuiles } & $\begin{array}{c}\text { Taille des } \\
\text { tegulae }\end{array}$ & $\begin{array}{c}\text { Nbre de } \\
\text { tuiles/m² }\end{array}$ & $\begin{array}{c}\text { masse au } \mathbf{~ m}^{\mathbf{2}} \\
\mathbf{( K g )}\end{array}$ \\
\hline Angers 'Saint-Louis', toiture effondrée 3206 & $360 \times 290$ & 12 & $\mathbf{7 1 , 2}$ \\
\hline Rezé, 'Rue Saint-Lupien', canalisation 8047 & $362 \times 278$ & 14 & $\mathbf{7 7 , 7}$ \\
\hline Angers 'Saint-Louis', canalisation 3703 & $421 \times 324$ & 9,5 & $\mathbf{8 7}$ \\
\hline Haute-Goulaine, 'Les Cléons', groupe 2 & $436 \times 336$ & 9 & $\mathbf{8 5 , 3}$ \\
\hline Jard-sur-Mer, Le Grand-Essart & $449 \times 361$ & 7,7 & $\mathbf{8 1 , 6}$ \\
\hline Angers 'Saint-Louis', us 3290-3291 & $525 \times 359$ & 6,9 & $\mathbf{9 2}$ \\
\hline
\end{tabular}

On peut poser deux types d'hypothèses quant au moteur de cette évolution. La première consiste à imaginer que l'on a souhaité réduire le poids des tegulae pour faciliter la manutention de ces matériaux sur les chantiers. On est passé en effet de tuiles approchant ou dépassant les dix kilos à l'unité sous Auguste à des tuiles de 4,5 kg en moyenne pour la période du III ${ }^{\mathrm{e}}$ siècle apr. J.-C. (ponctuellement jusqu'à $3,5 \mathrm{~kg}$ ). Dans ce cas, la baisse du poids total des toitures ne serait qu'une conséquence indirecte de cette volonté initiale de réduction du poids de chaque tegula.

La seconde hypothèse est centrée sur la volonté de réduction du poids de la toiture ellemême, par la réduction du poids des tuiles. L'intérêt d'une telle diminution se situe dans l'impact sur les charpentes et donc sur la structure même de celle-ci. Elle induit notamment une réduction des sections de bois de charpente et donc une diminution du coût global de la construction.

Pour une tegula, le gain de poids peut être principalement réalisé sur l'épaisseur du corps de la tuile (le gain réalisé sur la diminution de la largeur et la hauteur des rebords sera toujours plus modeste). Cependant, la diminution de l'épaisseur de la tuile induit une plus grande fragilité de celle-ci. Il est donc probable que c'est pour pallier cette fragilité potentielle que l'on a réduit également, en parallèle, les dimensions des tuiles, afin de maintenir une certaine robustesse de l'ensemble.

Il est difficile de trancher entre les deux hypothèses qui semblent cohérentes l'une et l'autre. Notons tout de même que la seconde s'accorde bien avec la tendance globale observée dans la construction romaine, qui va vers une économie généralisée des quantités de matériaux mis en œuvre (De Filippo, 2004, p. 103) ${ }^{17}$.

Cependant, ces hypothèses sont d'essence fonctionnaliste et il n'est pas interdit de faire intervenir davantage de facteurs culturels dans cette tentative de compréhension de l'évolution des tegulae entre le début de notre ère et le Bas Empire.

Il faut insister sur la rupture technique qu'a constituée l'introduction de la couverture romaine en Gaule du Nord au tout début du $\mathrm{I}^{\mathrm{er}}$ siècle apr. J.-C. Cette partie de la Gaule, 
qui n'avait connu que les couvertures végétales (chaume ou bardeaux) ${ }^{18}$ doit s'adapter à un matériau radicalement différent et qui induit la modification profonde de la structure des charpentes. La pente des toits passe de 40/50 degrés à environ 20 degrés, tandis que le poids des matériaux de couverture évolue quant à lui de 25/35 à 90/95 kg/ $\mathrm{m}^{2}$.

\section{Synthèse des résultats}

\section{Évolution morphologique}

En ce qui concerne la longueur des tuiles, l'évolution morphologique des tegulae est bien marquée (fig. 35) durant le $\mathrm{I}^{\mathrm{er}}$ siècle et probablement pendant une partie du $\mathrm{II}^{\mathrm{e}}$ siècle. Au-delà, les résultats sont plus délicats à interpréter, pour plusieurs raisons. D'une part, ce sont des horizons moins bien calés chronologiquement sur les sites, du fait d'un mobilier céramique moins datant. D'autre part, ils sont souvent moins présents. Dans certains de ces niveaux (III $\mathrm{e}^{\mathrm{e}} \mathrm{e}^{\mathrm{e}}$ siècles apr.J.-C.), des pratiques de remploi sont établies, ce qui en définitive ne permet pas de savoir si les éventuelles productions de ces périodes sont identiques à celles antérieures.

Figure 35 : Évolution du format des tegulae sur le site d'Angers "Saint-Louis ». Figure 35 : Evolution of tegulae size on the Angers "Saint-Louis" site.
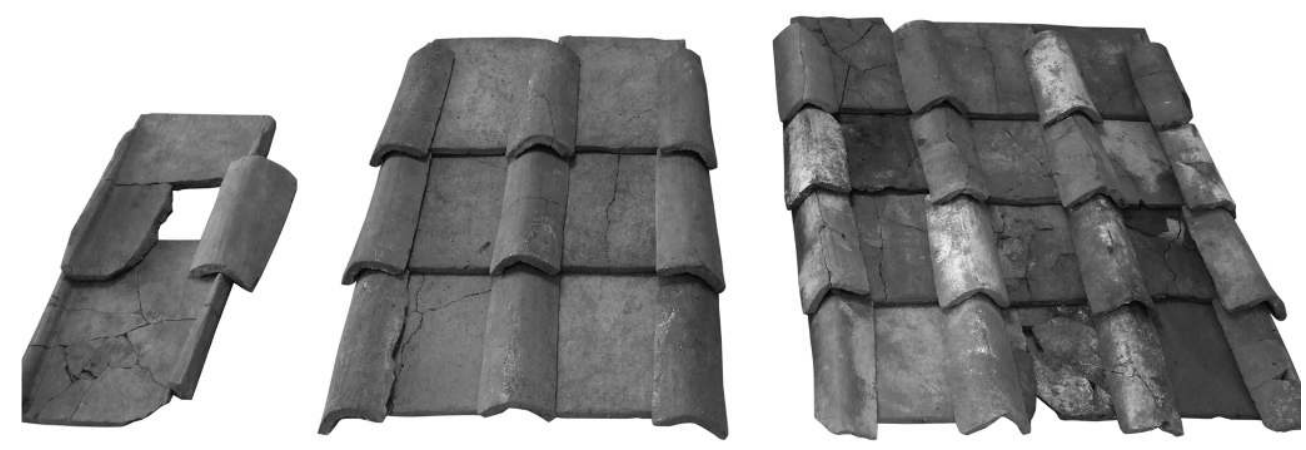

US 3291, première moitié du ^er siècle apr. J.-C., 525 x 359 mm. US 3703, fin ır siècle apr. J.-C., 421 x 324 mm. US 3206, début III siècle apr. J.-C., $360 \times 290$ mm.

US 3297, first half of the first century AD, $525 \times 359 \mathrm{~mm}$. US 3703, End of the first century $A D, 427 \times 324 \mathrm{~mm}$. US 3206, beginning of the third century AD, $360 \times 290 \mathrm{~mm}$.

Cliché J.-F. Nauleau, Inrap.

Parallèlement à cette diminution, maintenant bien documentée, on constate que la plupart des caractéristiques morphologiques de ces tuiles suivent une évolution identique. C'est le cas de l'épaisseur du corps des tuiles, de la largeur des rebords, de la longueur et de la largeur des encoches aval et amont ${ }^{19}$. Seule la hauteur des rebords varie peu.

Cependant, si la diminution globale de tous ces caractères est bien marquée, les valeurs constatées pour toutes ces données sont malgré tout dispersées. Il semble impossible d'estimer une longueur de tegula et donc de dater approximativement cette tuile, à partir d'une longueur d'encoche basse par exemple, sauf pour les valeurs extrêmes. Il paraît plus prudent de travailler sur plusieurs données en parallèle et de les combiner si l'on souhaite faire un travail de classement chronologique de tegulae à partir de leurs 
parties aval. Les études de pâte et l'étude des stigmates de façonnage sont également des données à corréler avec les précédentes.

Il faut d'autre part réaliser un travail statistique sur des effectifs importants et ne pas se contenter d'observations ponctuelles.

\section{Les tegulae peuvent-elles être considérées comme un matériau datant ?}

Les tegulae en tant que matériau de construction ne deviendront pas des éléments datant au même titre que les restes de poterie. En effet, n'étant pas des produits à forte valeur ajoutée, elles n'ont, en général ${ }^{20}$, pas été exportées loin de leur lieu de production. De ce fait, les référentiels d'évolution morphologique que l'on pourra réussir à construire ne seront valables que pour des aires géographiques restreintes et, qui plus est, difficile à circonscrire. En effet, s'il semble probable qu'on puisse définir des référentiels à l'échelle d'une ville, entité géographique et économique bien définie, cela est moins sûr pour les espaces ruraux ou par exemple le territoire de toute une cité. Il y a en effet nécessité de connaître les aires de diffusion des différents centres de production. On voit donc toute la difficulté à définir des courbes de références au-delà du seul cadre local.

Pour exemple, les formats des tegulae décrits dans le sud de la Gaule sont très différents de ceux observés ici. Si une évolution vers la réduction du format des tuiles a bien été esquissée (Feugère, 2000), les tailles sont bien différentes. Les plus petites tuiles rencontrées dans le sud de la Gaule au VI siècle apr. J.-C. correspondent presque à nos plus grandes tegulae du début $\mathrm{du} \mathrm{I}^{\mathrm{er}}$ siècle apr. J.-C. Il y a donc nécessité de connaître l'aire de validité d'un tel référentiel.

Rappelons enfin les limites méthodologiques de cette recherche. Si l'étude de corrélation entre les différents caractères de la tegula a porté sur plus de 170 individus, les effectifs ayant servi de support à l'examen de l'évolution morphologique de la longueur des tegulae sont plus restreints.

155 Gardons également à l'esprit les limites qui découlent de l'état de nos connaissances concernant la question des couvertures en terre cuite. On ne sait pas, à l'heure actuelle, si la couverture d'un bâtiment public mettait en œuvre exactement les mêmes matériaux que celle d'une domus, d'une villa ou que celle d'un bâtiment en matériaux périssables en milieu rural ${ }^{21}$.

156 Il n'est donc pas impossible que ce facteur puisse intervenir et perturber à la marge les observations qui ont été faites ici. L'étude des contextes architecturaux doit être réalisée autant que possible pour préciser ce point.

\section{Essor et déclin des tegulae dans l'aire d'étude}

\section{L'apparition des tuiles en Gaule}

La question de l'apparition de ces nouveaux matériaux de construction en Gaule est primordiale. On sait, en effet, que les premières toitures en tuile mises en œuvre dans le sud de la Gaule datent de la première moitié du $\mathrm{I}^{\mathrm{er}}$ siècle, voire du II siècle av. J.-C. La colonie grecque de Marseille produit même des tuiles dès la fin du vie siècle av. J.-C. 
(Clément, 2011), sur le modèle grec classique puis hellénistique. En revanche, pour la Gaule du Nord, les données sur l'apparition de ce matériau sont lacunaires.

À Angers, les tuiles apparaissent timidement dans les dernières décennies du $\mathrm{I}^{\mathrm{er}}$ siècle av. J.-C. (Mortreau, 2008). Des encoches aval atypiques ont été observées dans des niveaux augustéens sur la fouille du site de «Saint-Louis ». Elles sont dépourvues de réserve latérale et l'encoche est entièrement réalisée en face inférieure. Elles appartiennent à un type technique radicalement différent de celui composite classique observé en général dans la région.

En revanche, d'autres études mentionnent ce genre d'encoches aval dans le sud-est de la Gaule (Valence, Vienne et Lyon) pour des périodes de la seconde moitié du $\mathrm{I}^{\mathrm{er}}$ siècle av. J.-C. C'est également ce type d'encoche qui équipe les tegulae tardo-républicaines d'influence hellénistique sur le littoral de la Narbonnaise entre le $\mathrm{III}^{\mathrm{e}}$ et le $\mathrm{I}^{\mathrm{er}}$ siècle av. J.-C. (Clément, 2011).

160 Ces encoches sont également les premières à apparaître en Bretagne romaine (Warry, 2006), même si c'est de façon plus tardive (courant du $\mathrm{I}^{\mathrm{er}}$ siècle apr. J.-C.).

Il est probable que ces encoches singulières caractérisent les toutes premières tuiles produites (ou importées ?) à Angers vers le début de notre ère ou dans les dernières décennies $\mathrm{du} \mathrm{I}^{\mathrm{er}}$ siècle av. J.-C. Elles sont représentatives d'une culture technique dont il serait intéressant de connaître plus précisément l'origine. Cette dernière se situe vraisemblablement en Narbonnaise ou en Italie mais, en l'état actuel des connaissances, il est difficile d'aller plus loin.

\section{La transition vers le haut Moyen Âge}

La question de la transition vers le haut Moyen Âge est un sujet très peu documenté dans la région. Si ailleurs, en Languedoc, en Limousin et dans l'Orléanais (Chapelot, 2004), la production en contexte alto-médiéval est bien avérée, il n'en est rien ici.

Seul le site de la basilique paléochrétienne des «Champs Saint-Martin » à Rezé a livré en quantité importante des tegulae très probablement produites au début $\mathrm{du} \mathrm{VI}^{\mathrm{e}}$ siècle. En dehors de ce contexte, une seule tegula provenant des fouilles de l'église SaintSimilien à Nantes figure dans les collections du musée Dobrée (fig. 36). Notons enfin le lot en provenance de Carquefou (Nauleau, 2012), mais sans certitude quant au contexte chronologique de production. 
Figure 36 : Tegula du haut Moyen Âge (Tc 355, Nantes « Église Saint-Similien »). Figure 36 : Early Medieval Tegula (Tc 355, Nantes “Église Saint-Similien”).

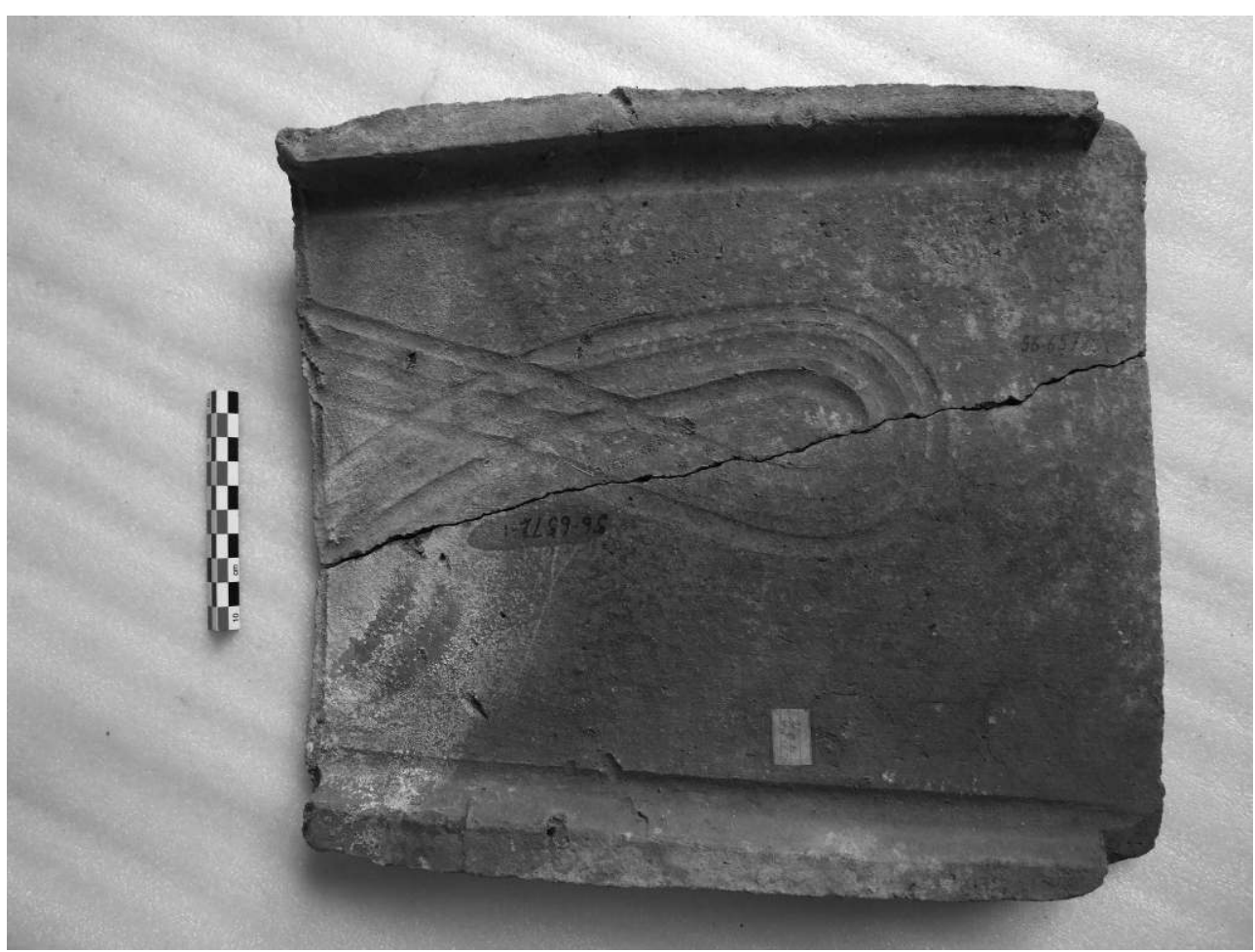

Cliché J.-F. Nauleau, Inrap.

Le format de ces tuiles est variable: l'exemplaire de Saint-Similien est très petit $(\mathrm{L}=343 \mathrm{~mm})$ tandis que les quelques tuiles archéologiquement complètes de Rezé s'échelonnent entre 380 et $405 \mathrm{~mm}$.

La tuile évolue vers un format plus trapézoïdal, alors que les tegulae romaines du HautEmpire sont le plus souvent parfaitement rectangulaires. L'exemplaire de SaintSimilien mesure $305 \mathrm{~mm}$ en partie haute et $30 \mathrm{~mm}$ de moins en partie basse. Une telle évolution a été notée dès l'Antiquité tardive (Angers « Saint-Louis »).

Les encoches d'emboîtement se sont largement réduites et semblent devenir inopérantes, le blocage de la tuile aval se faisant par le rebord de la tuile elle-même, grâce à sa forme en trapèze. Ainsi, à Rezé, l'encoche aval est quasi absente sur certains individus. La qualité du façonnage est médiocre, les rebords sont très irréguliers et les encoches très disparates d'un individu à l'autre.

167 Un point commun associe la tuile de Nantes et celle de Rezé : le type de pâte. Il s'agit dans les deux cas d'une argile très cuite, d'aspect grenu, comportant de nombreuses inclusions siliceuses, qui diffère sensiblement des pâtes fines orange-rouge antiques. Cette question mériterait de plus amples développements : il faudrait préciser si cette observation n'est valable que localement ou s'il s'agit d'une corrélation généralisable régionalement, car elle pourrait constituer un critère d'identification pour ces productions alto-médiévales.

168 En l'état actuel des connaissances, on peut estimer que, régionalement, la production de matériaux de construction en terre cuite est quasi nulle pendant le haut Moyen Âge. Elle semble limitée aux édifices cultuels, puisqu'en dehors des quelques matériaux de couverture cités, on peut recenser les remarquables briques moulées provenant 
principalement de la même église Saint-Similien à Nantes et de l'abbatiale Saint-JeanBaptiste de Vertou (Costa, 1964) ${ }^{22}$. Il s'agit donc d'une production très spécifique et qui semble ne plus concerner que de très rares édifices à cette époque.

La période charnière semble être l'Antiquité tardive, au cours de laquelle des mutations importantes se mettent en place, avec l'abandon de la plupart des techniques de construction d'origine romaine.

\section{Conclusion} culturels majeurs. 


\section{BIBLIOGRAPHIE}

ADAM J.-P., 2008 - La construction romaine, matériaux et techniques, Paris, Éditions Picard.

BAILlY P., 1981 - « Objets et documents archéologiques du site de Drevant conservés à Bourges $\left(1^{\text {re }}\right.$

partie) », Bulletin des amis du musée Saint-Vic (Saint-Amant-Montrond), 6 : 3-8.

BARRAUD D., 1979 - Fouille de sauvetage de la villa des Preux à Saint-Herblain (Loire-Atlantique), Rapport de fouilles, Direction des Antiquités, Nantes.

BOUET A., 1999 - Les matériaux de construction en terre cuite dans les thermes de la Gaule narbonnaise, Ausonius Publications, Bordeaux, (Scripta Antiqua, 1).

BOUET A., 2003 - Thermae Gallicae - Les thermes de Barzan (Charente-Maritime) et les thermes des provinces gauloises, Ausonius Publications, Bordeaux, (Supplément Aquitania, 11).

BouvET J.-P., 1987 - « Étude sur des briques de l'édifice de spectacle de Gennes-sur-Loire (49) », Journées archéologiques régionales « Matériaux de sol et de couverture », Direction des antiquités historiques des Pays de la Loire, Le Puy-du-Fou, 2 et 3 mai 1987.

CHAILlou F., 1884 - « Rapport sur les fouilles de la station gallo-romaine des Cléons », Bulletin de la société archéologique de Nantes et de la Loire-Inférieure, t. 23.

CHAILlou F., 1886 - « Collection locale des Cléons, Haute-Goulaine, près de Nantes. Rapport sur la découverte de cette station archéologique, description raisonnée des objets qui en proviennent ", Bulletin de la société archéologique de Nantes et de la Loire-Inférieure.

СНАРЕLот о., 2004 - « La terre cuite architecturale dans le bâtiment médiéval », in BESSAC J.-C. et al., La construction, les matériaux durs : pierre et terre cuite, Éditions Errance, Paris, 149-167.

CHAufin J., 1956 - « Les tuiles gallo-romaines du Bas-Dauphiné », Gallia, 14, nº 1, p. 81-88.

CHAUVET J.-Y., 1996 - Les toits des pays de France, Eyrolles, Paris.

CLEMENT B., 2009 - « Nouvelles données sur les tuiles de couverture en gaule du centre-est, de la fin de la république au III $^{\mathrm{e}}$ siècle », in Actes du congrès de Colmar, SFECAG, Marseille, 611-636.

CLEMENT B., 2011 - « La typologie des tuiles de terre cuite au deuxième âge du Fer en Gaule méridionale : nouvelles données ", in Actes du congrès d'Arles, SFECAG, Marseille, 597-606.

cosTA D., 1964 - Art mérovingien, Musée Th. Dobrée Nantes, Paris, (Inv. coll. publ. françaises, 10).

DESCHAMPS S., GUÉRIN F., PASCAL J. et PIRAULT L., 1992 - « Ratiatum (Rezé, Loire-Atlantique) : origines et développement de l'organisation urbaine », Revue archéologique de l'Ouest, nº 9, p. 111-127.

DE FILIPPO R., 2004 - « La brique et les matériaux de construction en terre cuite à l'époque romaine », in BESSAC J.-C. et al., La construction, les matériaux durs : pierre et terre cuite, Éditions Errance, Paris, p. 97-116.

DE FILIPPO R., 2010 - « Le remploi de la brique dans l'Antiquité tardive : le cas toulousain », Archéopages, $\mathrm{n}^{\circ} 29$, p. 38-47.

DEMANDRILle P. et CAMBou G., 2008 - Traité technique de couverture, Nouvelle édition, Ch. Massin et $C^{\text {ie }}$ éditeur, Paris.

eygun F., 1963 - «Circonscription de Poitiers », Gallia, 21, nº 2, p. 476 
FARAGo-SZEKERES B. et DUDAY H., 2008 - « Les tombes fastueuses de Naintré (Vienne) », in Les dossiers de l'archéologie, $\mathrm{n}^{\circ} 330$, p. 120-127.

FERDIERE A., 1988 - « Les dauphins en terre cuite de Briou « Moncelon » (Loir-et-Cher) : éléments de faîtage d'un édifice cultuel gallo-romain ? ", RACF, 27, 1, p. 115-122.

FEUGERE M., 2000 - « La longueur des tegulae : un indice chronologique ? » Instrumentum, $\mathrm{n}^{\circ} 11$, p. 24-25.

FINCKER M., 1986 - « Les briques-claveaux : un matériau de construction spécifique des thermes romains ", Aquitania, 4.

GIULIANI C.-F., 1990 - L'edilizia nell'Antichità, La Nuova Italia Scientifica, Rome.

GOULPEAU L., 1988 - « Introduction à une étude métrologique des tuiles et briques gallo-romaines », Revue archéologique de l'Ouest, $\mathrm{n}^{\circ}$ 5, p. 97-107.

GUITTON D., MONTEIL M., MOUCHARD J., DE PERETTI O. et RIEUNIER P., 2009 - L'agglomération antique de Rezé (LoireAtlantique), le quartier Saint-Lupien, Tome I - Fouille programmée 2006-2008, Rapport 2008 et bilan 2005-2008, université de Nantes, mairie de Rezé.

HERVE M.-L., 2007 - Piriac-sur-Mer (Loire-Atlantique), ZA du Pladreau, Rapport de fouille archéologique, Inrap GO, Cesson-Sévigné.

HERVE M.-L., 2008 - Le Langon (Vendée), Rue de l'industrie, Rapport de fouille archéologique, Inrap GO, Cesson-Sévigné.

ноғмANN B., 1975 - «Les matériaux de construction antiques en terre cuite », in « Céramique en Gaule romaine ", Les Dossiers de l'Archéologie, n 9, p. 111-120.

LAUBENHEIMER F., 1990 - Sallèles d'Aude. Un complexe de potiers gallo-romains : le quartier artisanal, Éditions de la maison des sciences de l'homme, Paris, Documents d'Archéologie française, 26.

LE вонес Y., 1983 - « Les marques sur briques de Jublains. Recherches de chronologie relative sur le complexe fortifié », in Armée romaine et province, III, p. 88-94.

LE вот A., 2003 - « Les thermes gallo-romains en Armorique », Les dossiers du CeRAA, Suppl., $\mathrm{n}^{\circ} \mathrm{Z}$.

LYoNNET c., 2008 - Les couvertures en tuiles, Centre scientifique et technique du bâtiment, Marne-laVallée.

MALIGORNE Y., CHEVET P. et FERRETTE R., 2010 - « DeuX maquettes architecturales récemment découvertes à Rennes », Latomus, 69, 1, p. 99-122.

MERCIER F., 2007 - Les Pellières Zac Ar Mor, Saint-Herblain (Loire-Atlantique), Rapport de fouille archéologique. Inrap GO, Cesson-Sévigné.

MORTREAU M., 2008 - « Indices de la présence de militaires romains à Angers, Luliomagus ( $\mathrm{I}^{\mathrm{er}}$ av.$\mathrm{III}^{\mathrm{e}}$ apr. J.-C.) », in Archives d'Anjou, 12, p. 9-39.

NAULEAU J.-F., $2009 \boldsymbol{a}$ - «Les matériaux de couverture en terre cuite », in VALAIS A., Précigné (Vendée), manoir du Plessis-Roland, Rapport de diagnostic archéologique, Inrap GO, Cesson-Sévigné.

NAULEAU J-F., 2009b - Jard-sur-Mer (Vendée), Le Grand Essart. Une villa gallo-romaine et une occupation de l'antiquité tardive et du haut Moyen Âge, Rapport de fouille archéologique, Inrap GO, CessonSévigné.

NAULEAU J-F., 2011 - « Les matériaux de construction en terre cuite », in DoYEN D., Saint-Michel-ChefChef, Loire-Atlantique, ZAC de La Pouplinière. Occupations littorales et ressources marines : du sel gaulois 
au colorant pourpre gallo-romain. Volume 3, annexes, Rapport de fouille archéologique, Inrap GO, Cesson-Sévigné.

NAULEAU J.-F., 2012 - «Les matériaux de construction en terre cuite », in VALAIS A., Carquefou (LoireAtlantique), ZAC de la Haute Forêt, tranche II, Rapport final d'opération, fouille archéologique, Inrap GO, Cesson-Sévigné : 244-249.

NAVEAU J., 1992 - « Les thermes d'Entrammes (Mayenne) », Revue archéologique de l'Ouest, $\mathrm{n}^{\circ} 9$, p. 129-159.

PIRAuLt L., 2009 - « La basilique des Champs Saint-Martin à Rezé (Loire-Atlantique) », in PARIS POULAIN D. (dir.), Les premiers temps chrétiens dans le territoire de la France actuelle, PUR, Rennes, p. 181-194.

PIтноN M., 2011 - Angers (Maine-et-Loire), Rue Chaussée Saint-Pierre. Archéologie d'une rue d'Angers,

Rapport de fouille archéologique, Inrap GO, Cesson-Sévigné.

PIтном м., 2012 - Angers (Maine-et-Loire), Place Marengo, aux marges de la ville antique, Rapport de fouille archéologique, Inrap GO, Cesson-Sévigné.

POoLE C., 2007 - Southampton French Quarter 1382, Specialist Report Download F5 : Ceramic building material. http://library.thehumanjourney.net/57/1/ SOU_1382_Specialist_report_download_F5.pdf. Consulté en avril 2011.

RIcco C., 1995 - « La diffusion par mer des matériaux de construction en terre cuite : un aspect mal connu du commerce antique en Méditerranée occidentale ", Mélanges de l'école française de Rome, Volume 107, $\mathrm{n}^{\circ}$ 2, p. 767-800.

SERIS D., 2008 - Les Clouzeaux (Vendée) La Grosse Pierre - un atelier gallo-romain de production de terres cuites architecturales et de céramiques, Rapport de fouille archéologique, Inrap GO, Cesson-Sévigné. TOURNEUR J., 2006 - Beaufort-en-Vallée (Maine-et-Loire), Chemin des Airaults, Rapport de diagnostic archéologique, Inrap GO, Cesson-Sévigné.

VIPARD P., 1999 - « Les matériaux architecturaux en terre cuite dans la domus du 'Bas de Vieux' », in SAN JUAN G. et MANEUVRIER J., L'exploitation ancienne des roches dans le Calvados : Histoire et Archéologie, Service départemental d'archéologie du Calvados, p. 209-219.

WARRY P., 2006 - « Tegulae, Manufacture, typology and use in Roman Britain », Archaeopress, Oxford. (BAR British Series, 417).

\section{NOTES}

1. Le site des Cléons à Haute-Goulaine (44) a été fouillé à la fin du $\mathrm{XIX}^{\mathrm{e}}$ siècle par F. Chaillou (Chaillou, 1884). Le mobilier provenant de cette fouille est géré par le musée départemental Thomas Dobrée à Nantes et compte un lot considérable de matériaux de construction en terre cuite.

2. Les termes " partie haute » et "partie basse » ou "partie amont» et "partie aval» seront utilisés pour décrire les différentes parties d'une tegula, en privilégiant la position de la tuile en œuvre sur la toiture. Les termes «avant » et « arrière » utilisés parfois, mais source de confusion, ne seront pas repris.

3. Le recouvrement des tegulae modernes et contemporaines employées dans toute l'Italie centrale est proche des $20 \mathrm{~cm}$. 
4. Les tuiles plates médiévales, non rencontrées sur l'aire d'étude (présentes en Sarthe et Mayenne) sont plus lourdes, en raison du mode de pose «tiercé » (3 épaisseurs), entre 70 et $75 \mathrm{~kg} / \mathrm{m}^{2}$ environ (Saint-Corneille (72), Nauleau dans Guérin, à paraître).

5. Le pureau, terme traditionnel, est la partie visible de la tuile après la pose.

6. Notons que l'usage de tegulae complètes ou fracturées en remplacement des briques est assez courant.

7. Antoine Archer (étudiant en master 2 à Nantes) a réalisé ce travail en 2011.

8. À Neuville-sur-Sarthe (72), sur la fouille du site du «Chapeau ", un caveau daté du III ou du IV siècle a fourni des briques très homogènes de format $320 \times 260 \mathrm{~mm}$ (G. Guillier, à paraître). Cette donnée pourrait confirmer régionalement la tendance à la réduction de la taille des briques.

9. Signalons également la présence dans ce lot de rarissimes quarts-de-rond taillés dans la roche locale, un falun peu induré.

10. Aucun exemple de pilette de section circulaire en briques n'est à mentionner dans l'aire d'étude.

11. Il s'agit de pilettes de renfort installées probablement après l'apparition de désordres dans la suspensura.

12. «Fragments de carreaux de cinq centimètres d'épaisseur, portant une échancrure semi-circulaire, faite avant la cuisson. Ils ont été trouvés parmi les débris d'un édifice dont ils formaient sans doute les jours. Réunis deux à deux, ils constituaient de petites ouvertures rondes (fenestrellae) de 0,18 $\mathrm{m}$ de diamètre, closes par des verres dont nous avons rencontrés les débris » (Chaillou, 1886, p. 31).

13. Les terres cuites ont été entièrement prélevées et identifiées. C'est l'absence totale d'imbrices au sein du lot alto-médiéval qui permet d'aboutir à cette conclusion.

14. Le décor cruciforme appliqué sur la tuile évoque certains registres rencontrés sur les céramiques décorées à la molette. Malgré le remploi avéré, il pourrait donc s'agir de productions alto-médiévales.

15. L'étude portant sur la Gaule du Centre-Est (Clément, 2009) montre une baisse nette de la longueur des tegulae. À la fin de la période républicaine, la longueur est de $57,5 \mathrm{~cm}$; au i ${ }^{\text {er }}$ siècle, elle est de $52 \mathrm{~cm}$, puis, pendant le II $^{\mathrm{e}}$ siècle, on passe de $46 / 49$ à $43 / 44 \mathrm{~cm}$. De la fin du $\mathrm{II}^{\mathrm{e}}$ jusqu'au IV ${ }^{\mathrm{e}}$ siècle, la longueur se stabilise à $42 / 43 \mathrm{~cm}$. En Grande-Bretagne romaine, Peter Warry montre clairement une baisse nette de la taille des tegulae entre le $\mathrm{I}^{\mathrm{er}}$ et le $\mathrm{III}^{\mathrm{e}}$ siècle apr. J.-C., qui s'accompagne d'une baisse de tous les autres caractères morphologiques de la tuile (rebords, encoches, épaisseur). Enfin, pour la domus du «Bas de Vieux » (Calvados: Vipard, 1999), la longueur des tuiles de type A de la fin du $\mathrm{II}^{\mathrm{e}}$ siècle varie entre 32 et $38 \mathrm{~cm}$ et pèse $5,2 \mathrm{~kg}$. Le type $B$ (début du $\mathrm{III}^{\mathrm{e}}$ siècle) est long de $33 / 34 \mathrm{~cm}$, les tuiles ne pèsent plus que $3 \mathrm{~kg}$ et deviennent trapézoïdales.

16. En revanche, la taille des tuiles se réduisant, leur nombre augmente, à surface égale : on passe ainsi de sept tegulae au mètre carré pour le début de notre ère (Angers "Saint-Louis ", US 3291) à treize au début ou milieu du ${ }^{\mathrm{e}}{ }^{\mathrm{e}}$ siècle (Angers "SaintLouis ", US 3206). Le gain de poids obtenu grâce à la diminution de l'épaisseur est ainsi atténué par une augmentation des surfaces "mortes»: augmentation des surfaces de recouvrement entre tuiles et du volume de rebords. 
17. La réduction de l'épaisseur des tuiles est aussi profitable à la phase de production : séchage plus rapide, cuisson plus aisée et augmentation de la quantité de tuiles contenues dans le laboratoire du four, à chaque fournée.

18. Notons tout de même que l'introduction des couvertures en tuile n'a probablement pas fait disparaitre totalement les couvertures végétales. Leur proportion relative était peut-être encore très importante, surtout en milieu rural. Une étude statistique visant à quantifier ces bâtiments de «tradition gauloise » en milieu gallo-romain est à mener régionalement.

19. En 1956, L. Chauffin, en précurseur, avait noté en Bas Dauphiné (Chauffin, 1956) une décroissance de la taille des encoches basses, qui passaient de $70 / 85 \mathrm{~mm}$ pour la période des $\mathrm{I}^{\mathrm{er}}-\mathrm{III}^{\mathrm{e}}$ siècles apr. $\mathrm{J}$.-C à $60 / 70 \mathrm{~mm}$ pour les $\mathrm{III}^{\mathrm{e}}-\mathrm{V}^{\mathrm{e}}$ siècles et $45 \mathrm{~mm}$ pour le haut Moyen Âge.

20. Il existe des exceptions notables connues en Méditerranée par transport maritime (Ricco, 1995).

21. B. Hofmann n'a pas noté de différences entre les tuiles provenant du temple et des habitats voisins sur le site de Genainville dans le Val-d'Oise (Hofmann, 1975).

22. Ces briques à décor historié sont de plusieurs types: briques-claveaux trapézoïdales, briques barlongues ou carrées composant probablement des frises. Les motifs sont assez variés : chrisme accompagné des lettres alpha et oméga, scène de la tentation avec Adam et Ève autour de l'arbre de la Tentation, lièvre poursuivi par un chien (lévrier probablement), ou bien encore animaux marins, réels ou imaginaires, se rapportant aux légendes de l'Antiquité (Costa, 1964).

\section{RÉSUMÉS}

Les matériaux de construction en terre cuite tiennent une place primordiale dans la construction gallo-romaine. Employés dans les maçonneries, dans les hypocaustes et sur les toitures, ils deviennent au cours du I ${ }^{\text {er }}$ siècle apr. J.-C. des matériaux incontournables. Cependant, les études les concernant sont très rares, et souvent seuls les matériaux de couverture (tegulae et imbrices) sont identifiés. Pourtant, un examen attentif révèle souvent la grande variabilité typologique des matériaux rencontrés. Si ceux associés aux thermes sont assez bien connus, une part non négligeable est constituée de matériaux autres, peu décrits dans les publications. Le but d'une telle étude est de faire progresser l'identification de ces éléments et la compréhension de leur contexte architectural. La mise en évidence d'une évolution morphologique des tegulae est maintenant acquise, même si elle reste à affiner et que son aire de validité reste à préciser. Elle montre l'évolution d'un matériau d'origine italique dans un environnement gallo-romain. L'étude menée ici du mode diffusion de ces nouveaux matériaux et de la culture technique associée, autour du changement d'ère, puis de leur effacement progressif durant l'Antiquité tardive et le haut Moyen Âge, apporte un éclairage qui dépasse la problématique des techniques de construction. Il apparaît ainsi, de façon plus générale, que les flux et reflux de l'usage de la terre cuite dans la construction sont de bons marqueurs témoignant de changements économiques et culturels importants. 
Terracotta building materials hold an important place in gallo-roman building. They are employed in masonry, hypocaust and roofs and become very common during the first century AD. Nevertheless, studies about them are rare, and very often only tiles (tegulae and imbrices) are detailed. However, scrupulous examination can show the wide range of terracotta material used. Those used in baths are well known, but most of them have a different use and are very rarely described in scientific studies. The purpose of the present paper is to make headway in the identification of these elements and in the understanding of their use in buildings. The evolution of the shape of roman tegulae is one of the most important results of this work, even if it is in a preliminary stage. The geographical area under study also needs to be defined more precisely. It shows the evolution of material of Italic origin in a gallo-roman context. The study developed here on the spread of new material and technical knowledge during the first century BC and then of its fading away during the end of the Antiquity and the early Middle Ages sheds new light on what is more than simple building techniques. It is above all obvious that in a more general way, the development and the abandon of the use of terracotta in building are good indicators of economical and cultural change.

Los materiales de construcción de terracota tienen un papel destacado en la construcción galoromana. Utilizados en albañilería, hipocausto y techos, se convierten en el siglo primero de nuestra era en materiales inevitables. Sin embargo, los estudios sobre ellos son muy raros, y a menudo sólo se identifican los materiales de cubierta (tegulae e imbrices). A pesar de ello, un examen cuidadoso revela a menudo la gran variabilidad tipológica de los materiales encontrados. Si los que se asocian a los baños son bastante conocidos, una parte significativa está hecha de otros materiales, poco descritos en la literatura. El propósito de este estudio es avanzar en la identificación de estos elementos y en la comprensión de su contexto arquitectónico. A pesar de que la evolución morfológica de los tegulae está ahora adquirida, queda por perfeccionar y su rango de validez queda por precisar. Muestra la evolución de un material de origen itálico en un ambiente galo-romano. El estudio realizado del modo de difusión de estos nuevos materiales y de la cultura tecnológica que los está asociada en torno al cambio de era, y luego de su desaparición progresiva durante la Antigüedad tardía y la temprana Edad Media, proporciona una visión que va más allá de la cuestión de las técnicas de construcción. Parece, pues, de modo más general, que los flujos y reflujos de la utilización de la terracota en la construcción son buenos marcadores que reflejan grandes cambios económicos y culturales.

\section{INDEX}

Keywords : roman period, early medieval, architecture, ceramic building material, tiles, brick, ridge-tile, antefix, tegulae mammatae, tubuli, hypocaust.

Mots-clés : époque romaine, haut Moyen Âge, architecture, matériaux de construction, tuile, brique, briques-claveaux, faîtage, antéfixe, tegulae mammatae , tubuli, hypocauste

Palabras claves : época romana, temprana Edad Media, arquitectura, materiales de construcción, tejas, ladrillos, ladrillos cuñas, cresta, tegulae mammatae, tubuli, hipocausto.

\section{AUTEUR}

\section{JEAN-FRANÇOIS NAULEAU}

INRAP - 4 rue du Tertre, 44477 Carquefou 